\title{
Synthesis of Perfluoroalkyl-Substituted Azines via Nucleophilic Substitution of Hydrogen with Perfluoroisopropyl Carbanions
}

\author{
Rafał Loska and Mieczysław Mąkosza* \\ Institute of Organic Chemistry, Polish Academy of Sciences \\ Kasprzaka 44/52, 01-224 Warsaw 42, Poland \\ icho-s@icho.edu.pl
}

\section{Supporting Information}

\section{$\underline{\text { Contents }}$}

General experimental methods.

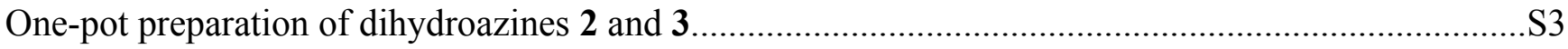

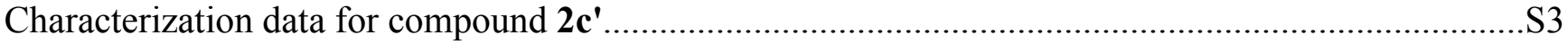

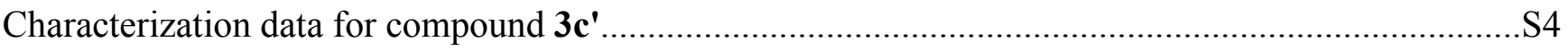

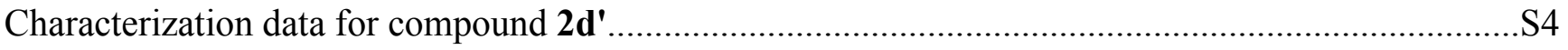

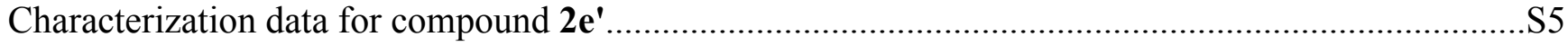

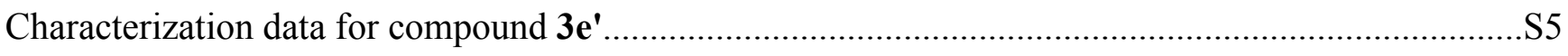

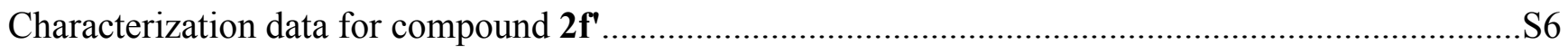

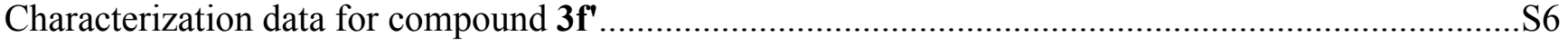

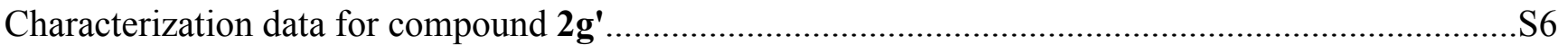

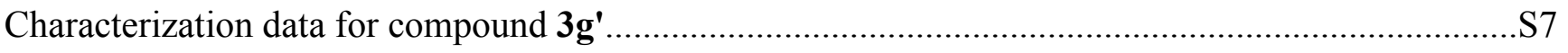

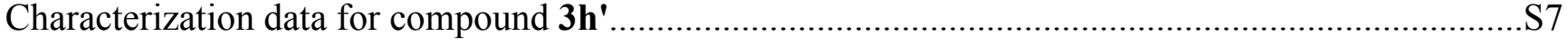

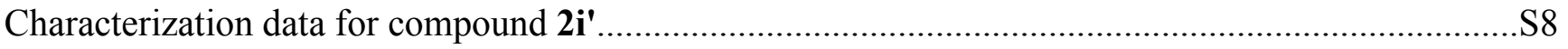

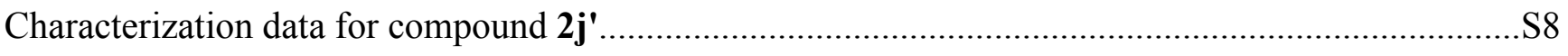

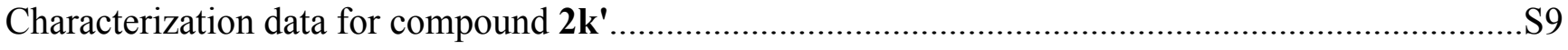

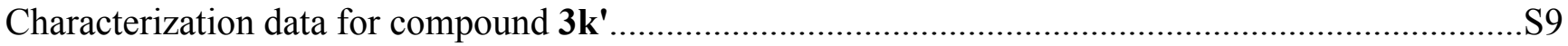

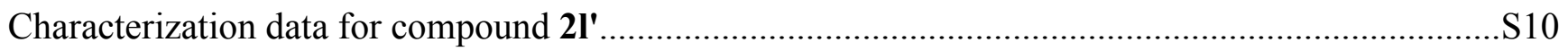




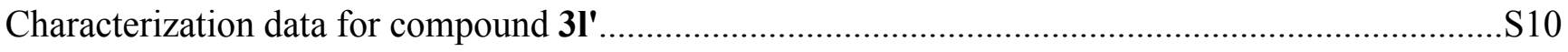

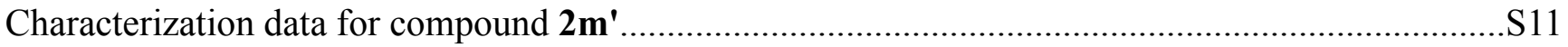

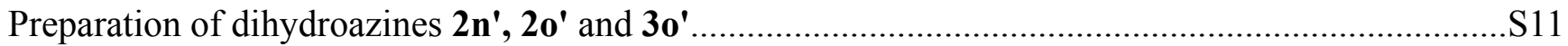

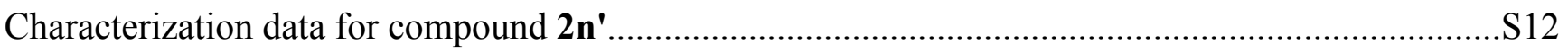

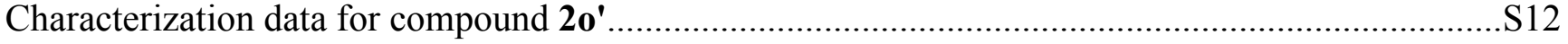

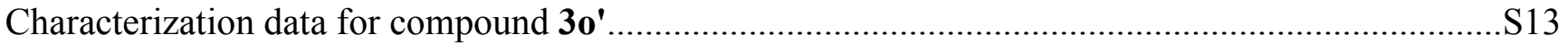

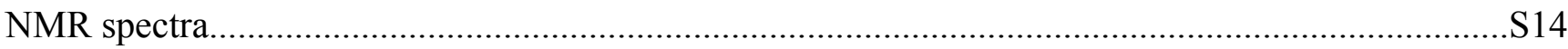

General experimental methods. The reactions with HFP and KF were performed under argon atmosphere (technical grade argon) in a flame dried glass pressure tube (c.a. $3.5 \mathrm{~mL}$ of volume) equipped with a teflon valve and a magnetic stirring element. All solvents used in these reactions were dried by standard techniques: DMF, MeCN, DME and $\mathrm{CH}_{2} \mathrm{Cl}_{2}$ were distilled from $\mathrm{CaH}_{2}$ and THF from potassium/benzophenone. ${ }^{1}$ Solvents used for extraction or chromatography were freshly distilled before use. Hunig's base $\left(i\right.$ - $\left.\mathrm{Pr}_{2} \mathrm{NEt}\right)$ was dried by storing over potassium hydroxide. Other reagents were used as obtained without further purification.

Flash chromatography was performed using silica gel $60(0.040-0.063 \mathrm{~mm})$ or neutral aluminium oxide. Thin layer chromatography was performed on pre-coated silica gel plates and visualized under a UV lamp.

NMR spectra were recorded in $\mathrm{CDCl}_{3}$ at the spectrometer frequencies indicated in the description of each compound. Chemical shifts are given in ppm relative to TMS for ${ }^{1} \mathrm{H}$ and ${ }^{13} \mathrm{C}$ NMR spectra and $\mathrm{CFCl}_{3}$ for ${ }^{19} \mathrm{~F}$ NMR spectra. IR spectra were obtained using a FT-IR spectrometer. Mass spectra were obtained using electron impact (EI) or electrospray (ESI) ionization.

$p$-Methoxybenzyl bromide was prepared according to a literature procedure. ${ }^{2}$

$N$-Alkylazinium salts are well known compounds and are usually prepared simply by heating an equimolar mixtutr of the azine and the alkylating agent. ${ }^{3}$ Salts $1 \mathbf{a}, \mathbf{1 c}$ and $\mathbf{1 d}$ were prepared in this way.

1 Armarego, W. F. L.; Perrin, D. D. Purification of Laboratory Chemicals; Butterworth-Heinemann: Oxford, 1996.

2 Ruder, S. M.; Ronald, R. C. Tetrahedron Lett. 1987, 28, 135.

3 See for example: (a) Cymerman Craig, J.; Garnett, J. L.; Temple, D. M. J. Chem. Soc. 1964, 4057. (b) Bunting, J. W.; Sindhuatmadja, S. J. Org. Chem. 1981, 46, 4211. (c) Fukuzumi, S.; Kommitsu, S.; Hironaka, K.; Tanaka, T. J. Am. Chem. Soc. 1987, 109, 305. (d) MacTavish, J.; Proctor, G. R.; Redpath, J. J. Chem. Soc., Perkin Trans. 1 1996, 2545. (e) Lo, H. C.; Buriez, O.; Kerr, J. B.; Fish, R. H. Angew. Chem. Int. Ed. 1999, 38, 1429. 
The most convenient way to obtain all other salts was to mix equimolar amounts of the azine and $\mathrm{BnBr}$ or $\mathrm{PMBBr}$ in acetone (in some cases toluene or $\mathrm{CHCl}_{3}$ ) at room temperature. After a few hours or days the crystalline salt was collected, washed and dried under vacuum. The idenity and purity of each salt was confirmed by its melting point, elemental analysis and ${ }^{1} \mathrm{H}$ NMR spectrum.

\section{General procedure of one-pot preparation of dihydroazines 2 and 3 directly from an azine and} PMBBr. An azine $(0.95 \mathrm{mmol})$ was dissolved in DMF $(2.75 \mathrm{~mL})$ in a glass pressure tube, then $\mathrm{PMBBr}$ (191 mg, $136 \mu \mathrm{L}, 0.95 \mathrm{mmol}$ ) was added and the tube was closed with a teflon valve. After stirring at room temperature for the time indicated in Table 1 (consumption of the azine was followed by TLC) the reaction mixture was cooled to $-75^{\circ} \mathrm{C}$ under argon atmosphere and HFP (c.a. $0.6 \mathrm{~g}, 4 \mathrm{mmol}$ ) was condensed into it (it is possible to measure approximately the volume of HFP already condensed since it is hardly miscible with DMF). Spray-dried $\mathrm{KF}_{(\mathrm{s})}(276 \mathrm{mg}, 4.75 \mathrm{mmol})$ was then added, the tube was closed again and its contents stirred vigorously for $3 \mathrm{~h}$ at room temperature. After opening of the tube the reaction mixture was poured into water $(10 \mathrm{~mL})$ and the products were extracted with $\mathrm{CH}_{2} \mathrm{Cl}_{2}(3 \times 5$ $\mathrm{mL})$. Combined organic layers were washed with water $(5 \times 10 \mathrm{~mL})$, dried over anhydrous $\mathrm{Na}_{2} \mathrm{SO}_{4}$ and concentrated. The products were purified by column chromatography on silica gel using 10:1 or 5:1 mixtures of hexanes/AcOEt as eluent (when two isomeric products were formed, mixtures of hexanes and $\mathrm{Et}_{2} \mathrm{O}$ of similar composition were sometimes more effective to separate them, see below).

2-Heptafluoroisopropyl-1-(p-methoxybenzyl)-1,2-dihydroquinoline (2c'). White solid, mp 63 - 63

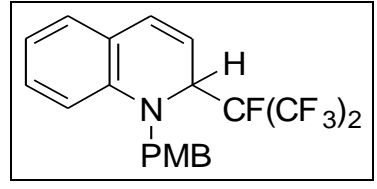
${ }^{\circ} \mathrm{C} . \mathrm{IR}\left(\mathrm{KBr}, v_{\max } / \mathrm{cm}^{-1}\right): 3039,2995,2838,1649,1602,1513,1497,1287$, 1258, 1233, 1149, 1029, 972, 952, 797, 761. ${ }^{1} \mathrm{H}$ NMR (400 MHz) $\delta=3.76$ $\left(3 \mathrm{H}, \mathrm{s}, \mathrm{OCH}_{3}\right), 4.38\left(1 \mathrm{H}, \mathrm{d},{ }^{2} J_{\mathrm{HH}}=15.5 \mathrm{~Hz}, \mathrm{NCH}_{2}\right), 4.91\left(1 \mathrm{H}, \mathrm{d},{ }^{2} J_{\mathrm{HH}}=15.7\right.$ $\left.\mathrm{Hz}, \mathrm{NCH}_{2}\right), 5.06(1 \mathrm{H}, \mathrm{m}, \mathrm{CHCF}), 5.56(1 \mathrm{H}, \mathrm{m}, \mathrm{CHCHCF}), 6.69(1 \mathrm{H}, \mathrm{m}$, $\mathrm{CH}=\mathrm{CHCHCF}), 6.69-6.74\left(2 \mathrm{H}, \mathrm{m}, \mathrm{H}_{\text {arom }}\right), 6.81\left(2 \mathrm{H}, \mathrm{dm},{ }^{3} J_{\mathrm{HH}}=8.7 \mathrm{~Hz}, \mathrm{PMB}\right), 6.99\left(1 \mathrm{H}, \mathrm{dd},{ }^{3} J_{\mathrm{HH}}=\right.$ $\left.7.4 \mathrm{~Hz},{ }^{4} J_{\mathrm{HH}}=1.8 \mathrm{~Hz}, \mathrm{H}_{\text {arom }}\right), 7.09\left(1 \mathrm{H}, \mathrm{td},{ }^{3} J_{\mathrm{HH}}=7.7 \mathrm{~Hz},{ }^{4} J_{\mathrm{HH}}=1.5 \mathrm{~Hz}, \mathrm{H}_{\text {arom }}\right), 7.12\left(2 \mathrm{H}, \mathrm{dm},{ }^{3} J_{\mathrm{HH}}=8.7\right.$ $\mathrm{Hz}, \mathrm{PMB}) .{ }^{13} \mathrm{C} \mathrm{NMR}(100 \mathrm{MHz}) \delta=55.2,55.4\left(\mathrm{~d},{ }^{4} J_{\mathrm{CF}}=4.3 \mathrm{~Hz}\right), 59.4\left(\mathrm{~d},{ }^{2} J_{\mathrm{CF}}=19.8 \mathrm{~Hz}\right), 92.1(\mathrm{dm}$, $\left.{ }^{1} J_{\mathrm{CF}}=206.4 \mathrm{~Hz},{ }^{2} J_{\mathrm{CF}}=29.1 \mathrm{~Hz}\right), 114.1,114.3\left(\mathrm{~d},{ }^{3} J_{\mathrm{CF}}=6.5 \mathrm{~Hz}\right), 114.5,118.5,120.7\left(\mathrm{qd},{ }^{1} J_{\mathrm{CF}}=287.4\right.$ $\left.\mathrm{Hz},{ }^{2} J_{\mathrm{CF}}=28.2 \mathrm{~Hz}\right), 120.8\left(\mathrm{qd},{ }^{1} J_{\mathrm{CF}}=287.7 \mathrm{~Hz},{ }^{2} J_{\mathrm{CF}}=26.3 \mathrm{~Hz}\right), 122.1,127.3,128.6,128.7,129.5$, 130.8, 143.1, 159.0. ${ }^{19} \mathrm{~F}$ NMR $(376 \mathrm{MHz}) \delta=-179.67\left(1 \mathrm{~F}, \mathrm{~m}, \mathrm{CF}\left(\mathrm{CF}_{3}\right)_{2}\right),-74.15\left(3 \mathrm{~F}, \mathrm{~m},{ }^{3} J_{\mathrm{FF}}=8.7 \mathrm{~Hz}\right.$, 
$\left.\mathrm{CF}\left(\mathrm{CF}_{3}\right)_{2}\right),-72.89\left(3 \mathrm{~F}, \mathrm{~m},{ }^{3} J_{\mathrm{FF}}=8.7 \mathrm{~Hz}, \mathrm{CF}\left(\mathrm{CF}_{3}\right)_{2}\right) . \mathrm{MS}(\mathrm{EI} 70 \mathrm{eV}) \mathrm{m} / z(\%): 419\left(\mathrm{M}^{+}, 2\right), 250(26)$, 121(100). HRMS (EI) : calcd for $\mathrm{C}_{20} \mathrm{H}_{16} \mathrm{NOF}_{7}\left(\mathrm{M}^{+}\right)$419.1120, found 419.1131. Anal. Calcd for $\mathrm{C}_{20} \mathrm{H}_{16} \mathrm{NOF}_{7}$ : C, 57.29; H, 3.85; N, 3.34; F, 31.71. Found : C, 57.93; H, 3.69; N, 3.04; F, 31.75.

4-Heptafluoroisopropyl-1-(p-methoxybenzyl)-1,4-dihydroquinoline (3c'). White solid, mp 57 - 58

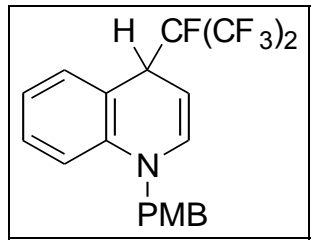

${ }^{\circ} \mathrm{C}$. IR $\left(\mathrm{CH}_{2} \mathrm{Cl}_{2}, v_{\max } / \mathrm{cm}^{-1}\right): 2934,1667,1514,1494,1467,1385,1295,1249$, 1222, 1178, 1109, 1038, 976, 752, 710. ${ }^{1} \mathrm{H}$ NMR $(400 \mathrm{MHz}) \delta=3.79(3 \mathrm{H}, \mathrm{s}$, $\left.\mathrm{OCH}_{3}\right), 4.55\left(1 \mathrm{H}, \mathrm{ddm},{ }^{3} J_{\mathrm{HH}}=7.8 \mathrm{~Hz}, 5.5 \mathrm{~Hz}, \mathrm{NCH}=\mathrm{CH}\right), 4.67\left(2 \mathrm{H}, \mathrm{AB},{ }^{2} J_{\mathrm{HH}}=\right.$ $\left.16.5 \mathrm{~Hz}, \mathrm{NCH}_{2}\right), 4.65-4.72(1 \mathrm{H}, \mathrm{m}, \mathrm{CHCF}), 6.39\left(1 \mathrm{H}, \mathrm{dd},{ }^{3} J_{\mathrm{HH}}=8.0 \mathrm{~Hz},{ }^{4} J_{\mathrm{HH}}=\right.$ $1.8 \mathrm{~Hz}, \mathrm{NCH}=\mathrm{CH}), 6.71\left(1 \mathrm{H}, \mathrm{dd},{ }^{3} J_{\mathrm{HH}}=8.4 \mathrm{~Hz},{ }^{4} J_{\mathrm{HH}}=1.0 \mathrm{~Hz}, \mathrm{H}_{\text {arom }}\right), 6.86(2 \mathrm{H}$, $\left.\mathrm{dm},{ }^{3} J_{\mathrm{HH}}=8.8 \mathrm{~Hz}, \mathrm{PMB}\right), 6.92\left(1 \mathrm{H}, \mathrm{td},{ }^{3} J_{\mathrm{HH}}=7.5 \mathrm{~Hz},{ }^{4} J_{\mathrm{HH}}=1.2 \mathrm{~Hz}, \mathrm{H}_{\text {arom }}\right), 7.11-7.19\left(4 \mathrm{H}, \mathrm{m}, \mathrm{H}_{\text {arom }}\right)$. ${ }^{13} \mathrm{C}$ NMR $(100 \mathrm{MHz}) \delta=40.7\left(\mathrm{~d},{ }^{2} J_{\mathrm{CF}}=20.7 \mathrm{~Hz}\right), 53.8,55.3,89.2\left(\mathrm{~d},{ }^{3} J_{\mathrm{CF}}=8.6 \mathrm{~Hz}\right), 89.0-92.5(\mathrm{~m})$, $112.8,114.2,114.4,116.5-126.0(\mathrm{~m}), 121.1,127.6,128.7,129.0,131.7$ (d, $\left.{ }^{3} J_{\mathrm{CF}}=4.2 \mathrm{~Hz}\right), 136.5$, 141.0, 158.9. ${ }^{19} \mathrm{~F}$ NMR $(376 \mathrm{MHz}) \delta=-174.50\left(1 \mathrm{~F}, \mathrm{~m}, \mathrm{CF}\left(\mathrm{CF}_{3}\right)_{2}\right),-74.05\left(3 \mathrm{~F}, \mathrm{~m}, \mathrm{CF}(\mathrm{CF})_{2}\right),-72.06$ $\left(3 \mathrm{~F}, \mathrm{~m}, \mathrm{CF}\left(\mathrm{CF}_{3}\right)_{2}\right)$. MS (EI $\left.70 \mathrm{eV}\right) \mathrm{m} / z(\%): 419\left(\mathrm{M}^{+}, 1\right), 297(1), 250(21), 121(100)$. HRMS (EI) : calcd for $\mathrm{C}_{20} \mathrm{H}_{16} \mathrm{NOF}_{7}\left(\mathrm{M}^{+}\right)$419.1120, found 419.1115. Anal. Calcd for $\mathrm{C}_{20} \mathrm{H}_{16} \mathrm{NOF}_{7}$ : C, 57.29; $\mathrm{H}, 3.85 ; \mathrm{N}$, 3.34; F, 31.71. Found : C, 57.15; H, 3.77; N, 3.17; F, 31.68.

1-Heptafluoroisopropyl-2-(p-methoxybenzyl)-1,2-dihydroisoquinoline (2d'). Pale yellow oil,

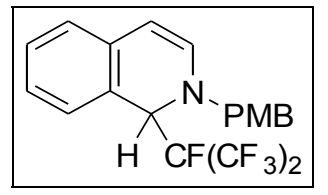

becomes dark-red after a few days at RT. IR (film, $\left.v_{\max } / \mathrm{cm}^{-1}\right)$ : 3064, 3031, 2937, 2839, 1626, 1513, 1288, 1228, 1157, 1112, 1036, 983, 770. ${ }^{1} \mathrm{H}$ NMR (400 MHz) $\delta=3.73\left(3 \mathrm{H}, \mathrm{s}, \mathrm{OCH}_{3}\right), 4.38\left(2 \mathrm{H}, \mathrm{s}, \mathrm{NCH}_{2}\right), 5.50\left(1 \mathrm{H}, \mathrm{d},{ }^{3} J_{\mathrm{HH}}=7.3 \mathrm{~Hz}\right.$, $\mathrm{NCH}=\mathrm{CH}), 5.62\left(1 \mathrm{H}, \mathrm{d},{ }^{3} J_{\mathrm{HF}}=7.2 \mathrm{~Hz}, \mathrm{CHCF}\right), 6.21\left(1 \mathrm{H}, \mathrm{dd},{ }^{3} J_{\mathrm{HH}}=7.3 \mathrm{~Hz},{ }^{4} J_{\mathrm{HH}}\right.$ $=1.2 \mathrm{~Hz}, \mathrm{NCH}=\mathrm{CH}), 6.77\left(2 \mathrm{H}, \mathrm{dm},{ }^{3} \mathrm{~J}_{\mathrm{HH}}=8.8 \mathrm{~Hz}, \mathrm{PMB}\right), 6.95\left(1 \mathrm{H}, \mathrm{d},{ }^{3} J_{\mathrm{HH}}=7.8 \mathrm{~Hz}, \mathrm{H}_{\text {arom }}\right), 6.98-$ $7.13\left(4 \mathrm{H}, \mathrm{m}, \mathrm{H}_{\text {arom }}\right), 7.23\left(1 \mathrm{H}, \mathrm{td},{ }^{3} J_{\mathrm{HH}}=7.5 \mathrm{~Hz},{ }^{4} J_{\mathrm{HH}}=1.4 \mathrm{~Hz}, \mathrm{H}_{\text {arom }}\right) .{ }^{13} \mathrm{C} \mathrm{NMR}(100 \mathrm{MHz}) \delta=55.1$, $58.9\left(\mathrm{~d},{ }^{4} J_{\mathrm{CF}}=2.6 \mathrm{~Hz}\right), 61.3\left(\mathrm{~d},{ }^{2} J_{\mathrm{CF}}=22.3 \mathrm{~Hz}\right), 90.5\left(\mathrm{dm},{ }^{1} J_{\mathrm{CF}}=206.0 \mathrm{~Hz},{ }^{2} J_{\mathrm{CF}}=30.2 \mathrm{~Hz}\right), 101.8$, $114.1,118.8\left(\mathrm{~d},{ }^{4} J_{\mathrm{CF}}=2.6 \mathrm{~Hz}\right), 120.9\left(\mathrm{qd},{ }^{1} J_{\mathrm{CF}}=289.6 \mathrm{~Hz},{ }^{2} J_{\mathrm{CF}}=27.6 \mathrm{~Hz}\right), 121.0\left(\mathrm{qd},{ }^{1} J_{\mathrm{CF}}=285.3 \mathrm{~Hz}\right.$, $\left.{ }^{2} J_{\mathrm{CF}}=27.6 \mathrm{~Hz}\right), 123.5,125.2,128.2,128.5,128.9,129.4,134.1,135.2,159.1 .{ }^{19} \mathrm{~F} \mathrm{NMR}(376 \mathrm{MHz}) \delta=$ $-178.85\left(1 \mathrm{~F}, \mathrm{~m},{ }^{3} J=6.7 \mathrm{~Hz}, \mathrm{CF}\left(\mathrm{CF}_{3}\right)_{2}\right),-72.33\left(3 \mathrm{~F}, \mathrm{~m},{ }^{3} J_{\mathrm{FF}}=9.4 \mathrm{~Hz}, \mathrm{CF}\left(\mathrm{CF}_{3}\right)_{2}\right),-72.04\left(3 \mathrm{~F}, \mathrm{~m},{ }^{3} J_{\mathrm{FF}}=\right.$ 8.0 Hz, $\left.\mathrm{CF}\left(\mathrm{CF}_{3}\right)_{2}\right) . \mathrm{MS}(\mathrm{EI} 70 \mathrm{eV}) \mathrm{m} / z(\%): 419\left(\mathrm{M}^{+}, 4\right), 250(36), 121(100)$. HRMS (EI) : calcd for $\mathrm{C}_{20} \mathrm{H}_{16} \mathrm{NOF}_{7}\left(\mathrm{M}^{+}\right)$419.1120, found 419.1140. Anal. Calcd for $\mathrm{C}_{20} \mathrm{H}_{16} \mathrm{NOF}_{7}: \mathrm{C}, 57.29 ; \mathrm{H}, 3.85 ; \mathrm{N}, 3.34$. 
Found : C, 56.99; H, 3.65; N, 3.29\%.

2-Heptafluoroisopropyl-1-(p-methoxybenzyl)-5-methoxycarbonyl-1,2-dihydropyridine

$\left(2 \mathrm{e}^{\prime}\right)$.

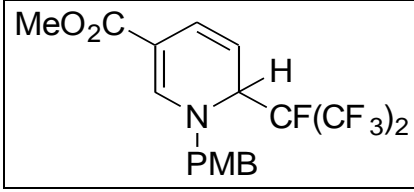

Separated from $3 \mathbf{e}^{\prime}$ by chromatography using 10:1 hexanes/ $\mathrm{Et}_{2} \mathrm{O}$, pale yellow oil. IR $\left(\mathrm{CH}_{2} \mathrm{Cl}_{2}, v_{\max } / \mathrm{cm}^{-1}\right): 3002,2954,2841,1698,1642,1577$, $1514,1440,1290,1253,1226,1141,1109,744 .{ }^{1} \mathrm{H}$ NMR $(400 \mathrm{MHz}) \delta=$ $3.72\left(3 \mathrm{H}, \mathrm{s}, \mathrm{OCH}_{3}\right), 3.81\left(3 \mathrm{H}, \mathrm{s}, \mathrm{OCH}_{3}\right), 4.48\left(2 \mathrm{H}, \mathrm{AB},{ }^{2} J_{\mathrm{HH}}=16.2\right.$, $\left.\mathrm{NCH}_{2}\right), 4.89(1 \mathrm{H}, \mathrm{m}, \mathrm{CHCHCF}), 5.05(1 \mathrm{H}, \mathrm{m}, \mathrm{CHCF}), 6.70\left(1 \mathrm{H}, \mathrm{d},{ }^{3} J_{\mathrm{HH}}=9.8 \mathrm{~Hz}, \mathrm{CH}=\mathrm{CHCHCF}\right)$, $6.90\left(2 \mathrm{H}, \mathrm{d},{ }^{3} J_{\mathrm{HH}}=8.7 \mathrm{~Hz}, \mathrm{PMB}\right), 7.16\left(2 \mathrm{H}, \mathrm{d},{ }^{3} J_{\mathrm{HH}}=8.7 \mathrm{~Hz}, \mathrm{PMB}\right), 7.47\left(1 \mathrm{H}, \mathrm{d},{ }^{4} J_{\mathrm{HH}}=0.6 \mathrm{~Hz}\right.$, $\left.\mathrm{NCH}=\mathrm{CCO}_{2} \mathrm{Me}\right) .{ }^{13} \mathrm{C} \mathrm{NMR}(100 \mathrm{MHz}) \delta=51.3,55.5,58.6\left(\mathrm{~d},{ }^{2} J_{\mathrm{CF}}=19.0 \mathrm{~Hz}\right), 60.4\left(\mathrm{~d},{ }^{4} J_{\mathrm{CF}}=6.0 \mathrm{~Hz}\right)$, $92.0\left(\mathrm{dm},{ }^{1} J_{\mathrm{CF}}=207.8 \mathrm{~Hz},{ }^{2} J_{\mathrm{CF}}=28.4 \mathrm{~Hz}\right), 101.4,103.7\left(\mathrm{dm},{ }^{3} J_{\mathrm{CF}}=7.8 \mathrm{~Hz},{ }^{4} J_{\mathrm{CF}}=1.7 . \mathrm{Hz}\right), 114.8$, $120.6\left(\mathrm{qd},{ }^{1} J_{\mathrm{CF}}=288.0 \mathrm{~Hz},{ }^{2} J_{\mathrm{CF}}=26.7 \mathrm{~Hz}\right), 120.8\left(\mathrm{qd},{ }^{1} J_{\mathrm{CF}}=287.9 \mathrm{~Hz},{ }^{2} J_{\mathrm{CF}}=28.4 \mathrm{~Hz}\right), 127.0,127.5$, 129.4, 147.0, 160.0, 166.4. ${ }^{19} \mathrm{~F}$ NMR (376 MHz) $\delta=-177.47\left(1 \mathrm{~F}, \mathrm{~m}, \mathrm{CF}\left(\mathrm{CF}_{3}\right)_{2}\right),-73.75(3 \mathrm{~F}, \mathrm{~m}$, $\left.\mathrm{CF}\left(\mathrm{CF}_{3}\right)_{2}\right)$, -72.66 (3F, m, $\left.\mathrm{CF}\left(\mathrm{CF}_{3}\right)_{2}\right)$. HRMS (ESI, MeOH) : calcd for $\mathrm{C}_{18} \mathrm{H}_{16} \mathrm{NO}_{3} \mathrm{~F}_{7} \mathrm{Na}\left(\mathrm{M}+\mathrm{Na}^{+}\right)$ 450.0911, found 450.0913. Anal. Calcd for $\mathrm{C}_{18} \mathrm{H}_{16} \mathrm{NO}_{3} \mathrm{~F}_{7}: \mathrm{C}, 50.59 ; \mathrm{H}, 3.77 ; \mathrm{N}, 3.28 ; \mathrm{F}, 31.12$. Found : C, 50.53; H, 3.72; N, 3.42; F, 31.18.

4-Heptafluoroisopropyl-1-(p-methoxybenzyl)-3-methoxycarbonyl-1,4-dihydropyridine

$\left(3 \mathrm{e}^{\prime}\right)$.

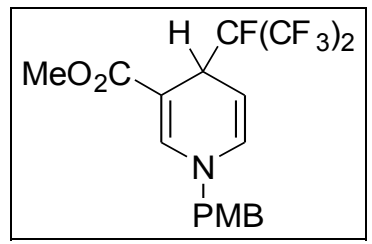

Separated from $2 e^{\prime}$ by chromatography using 10:1 hexanes/ $\mathrm{Et}_{2} \mathrm{O}$, pale yellow oil. IR (film, $v_{\max } / \mathrm{cm}^{-1}$ ) : 3003, 2954, 2841, 1704, 1590, 1515, 1415, 1304, 1254, 1217, 1169, 1086, 977, 789, 749, 723. ${ }^{1} \mathrm{H}$ NMR $(500 \mathrm{MHz}) \delta=3.71$ $\left(3 \mathrm{H}, \mathrm{s}, \mathrm{OCH}_{3}\right), 3.80\left(3 \mathrm{H}, \mathrm{s}, \mathrm{OCH}_{3}\right), 4.40\left(2 \mathrm{H}, \mathrm{s}, \mathrm{NCH}_{2}\right), 4.70(1 \mathrm{H}, \mathrm{m}, \mathrm{CHCF})$, $4.77\left(1 \mathrm{H}, \mathrm{dd},{ }^{3} J_{\mathrm{HH}}=7.0 \mathrm{~Hz},{ }^{3} J_{\mathrm{HH}}=6.3 \mathrm{~Hz}, \mathrm{NCH}=\mathrm{CH}\right), 6.15\left(1 \mathrm{H}, \mathrm{d},{ }^{3} J_{\mathrm{HH}}=7.7 \mathrm{~Hz}, \mathrm{NCH}=\mathrm{CH}\right), 6.89$ $\left(2 \mathrm{H}, \mathrm{d},{ }^{3} J_{\mathrm{HH}}=8.6 \mathrm{~Hz}, \mathrm{PMB}\right), 7.12\left(2 \mathrm{H}, \mathrm{d},{ }^{3} J_{\mathrm{HH}}=8.6 \mathrm{~Hz}, \mathrm{PMB}\right), 7.45\left(1 \mathrm{H}, \mathrm{d},{ }^{4} J_{\mathrm{HH}}=1.1 \mathrm{~Hz}\right.$, $\left.\mathrm{NCH}=\mathrm{CCO}_{2} \mathrm{Me}\right) .{ }^{13} \mathrm{C} \mathrm{NMR}(125 \mathrm{MHz}) \delta=35.3\left(\mathrm{~d},{ }^{2} J_{\mathrm{CF}}=21.4 \mathrm{~Hz}\right), 51.2,55.2,57.5,91.3\left(\mathrm{dm},{ }^{1} J_{\mathrm{CF}}=\right.$ $\left.207.9 \mathrm{~Hz},{ }^{2} J_{\mathrm{CF}}=28.3 \mathrm{~Hz}\right), 93.4\left(\mathrm{~d},{ }^{3} J_{\mathrm{CF}}=4.6 \mathrm{~Hz}\right), 97.5\left(\mathrm{~d},{ }^{3} J_{\mathrm{CF}}=6.1 \mathrm{~Hz}\right), 114.3,120.9\left(\mathrm{qd},{ }^{1} J_{\mathrm{CF}}=288.1\right.$ $\left.\mathrm{Hz},{ }^{2} J_{\mathrm{CF}}=28.4 \mathrm{~Hz}\right), 121.3\left(\mathrm{qd},{ }^{1} J_{\mathrm{CF}}=287.5 \mathrm{~Hz},{ }^{2} J_{\mathrm{CF}}=27.8 \mathrm{~Hz}\right), 127.8,128.8,132.3,143.0,159.6$, 168.2. ${ }^{19} \mathrm{~F}$ NMR $(376 \mathrm{MHz}) \delta=-184.11\left(1 \mathrm{~F}, \mathrm{~m},{ }^{3} J=6.0 \mathrm{~Hz}, \mathrm{CF}\left(\mathrm{CF}_{3}\right)_{2}\right),-75.81\left(3 \mathrm{~F}, \mathrm{~m},{ }^{3} J_{\mathrm{FF}}=8.3 \mathrm{~Hz}\right.$ $\left.\mathrm{CF}\left(\mathrm{CF}_{3}\right)_{2}\right),-73.29\left(3 \mathrm{~F}, \mathrm{~m},{ }^{3} \mathrm{~J}_{\mathrm{FF}}=8.3 \mathrm{~Hz}, \mathrm{CF}\left(\mathrm{CF}_{3}\right)_{2}\right)$. HRMS (ESI, MeOH) : calcd for $\mathrm{C}_{18} \mathrm{H}_{16} \mathrm{NO}_{3} \mathrm{~F}_{7} \mathrm{Na}$ $\left(\mathrm{M}+\mathrm{Na}^{+}\right)$450.0911, found 450.0926. Anal. Calcd for $\mathrm{C}_{18} \mathrm{H}_{16} \mathrm{NO}_{3} \mathrm{~F}_{7}: \mathrm{C}, 50.59 ; \mathrm{H}, 3.77 ; \mathrm{N}, 3.28 ; \mathrm{F}$, 31.12. Found : C, 50.66; H, 3.79; N, 3.40; F, 29.87. 
3-Cyano-6-heptafluoroisopropyl-1-(p-methoxybenzyl)-1,6-dihydropyridine (2f'). Pale yellow solid, $\underbrace{\mathrm{NC}}_{\substack{1 \\ \mathrm{PMB}}}<_{\mathrm{CF}\left(\mathrm{CF}_{3}\right)_{2}}^{\mathrm{H}}$ $\operatorname{mp} 50-51{ }^{\circ} \mathrm{C}$. IR $\left(\mathrm{KBr}, v_{\max } / \mathrm{cm}^{-1}\right): 3064,3013,2968,2835,2204,1650$, 1582, 1514, 1385, 1295, 1253, 1223, 1109, 1032, 957, 750. ${ }^{1} \mathrm{H}$ NMR (500 $\mathrm{MHz}) \delta=3.82\left(3 \mathrm{H}, \mathrm{s}, \mathrm{OCH}_{3}\right), 4.43\left(2 \mathrm{H}, \mathrm{AB},{ }^{2} J_{\mathrm{HH}}=14.8 \mathrm{~Hz}, \mathrm{NCH}_{2}\right), 4.94$ $(1 \mathrm{H}, \mathrm{m}, \mathrm{CHCHCF}), 5.09(1 \mathrm{H}, \mathrm{m}, \mathrm{CHCF}), 6.21\left(1 \mathrm{H}, \mathrm{d},{ }^{3} J_{\mathrm{HH}}=9.7 \mathrm{~Hz}\right.$, $\mathrm{CH}=\mathrm{CHCHCF}), 6.92\left(2 \mathrm{H}, \mathrm{d},{ }^{3} J_{\mathrm{HH}}=8.6 \mathrm{~Hz}, \mathrm{PMB}\right), 6.98(1 \mathrm{H}, \mathrm{s}, \mathrm{NCH}=\mathrm{CCN}), 7.15\left(2 \mathrm{H}, \mathrm{d},{ }^{3} J_{\mathrm{HH}}=8.6\right.$ $\mathrm{Hz}, \mathrm{PMB}) .{ }^{13} \mathrm{C}$ NMR $(125 \mathrm{MHz}) \delta=55.2,58.2\left(\mathrm{~d},{ }^{2} J_{\mathrm{CF}}=19.8 \mathrm{~Hz}\right), 60.2\left(\mathrm{~d},{ }^{4} J_{\mathrm{CF}}=6.2 \mathrm{~Hz}\right), 81.9,91.5$ $\left(\mathrm{dm},{ }^{1} J_{\mathrm{CF}}=209.2 \mathrm{~Hz},{ }^{2} J_{\mathrm{CF}}=29.8 \mathrm{~Hz}\right), 105.2\left(\mathrm{~d},{ }^{3} J_{\mathrm{CF}}=7.5 \mathrm{~Hz}\right), 114.6,119.2,120.2\left(\mathrm{qd},{ }^{1} J_{\mathrm{CF}}=287.8 \mathrm{~Hz}\right.$, $\left.{ }^{2} J_{\mathrm{CF}}=27.8 \mathrm{~Hz}\right), 120.4\left(\mathrm{qd},{ }^{1} J_{\mathrm{CF}}=287.2 \mathrm{~Hz},{ }^{2} J_{\mathrm{CF}}=28.5 \mathrm{~Hz}\right), 125.8,126.5,129.4,147.7,160.0 .{ }^{19} \mathrm{~F} \mathrm{NMR}$ $(376 \mathrm{MHz}) \delta=-178.04\left(1 \mathrm{~F}, \mathrm{~m}, \mathrm{CF}\left(\mathrm{CF}_{3}\right)_{2}\right),-73.84\left(3 \mathrm{~F}, \mathrm{~m}, \mathrm{CF}\left(\mathrm{CF}_{3}\right)_{2}\right),-72.76\left(3 \mathrm{~F}, \mathrm{~m}, \mathrm{CF}(\mathrm{CF})_{2}\right)$. HRMS (ESI, MeOH) : calcd for $\mathrm{C}_{17} \mathrm{H}_{13} \mathrm{~N}_{2} \mathrm{OF}_{7} \mathrm{Na}\left(\mathrm{M}+\mathrm{Na}^{+}\right)$417.0808, found 417.0829. Anal. Calcd for $\mathrm{C}_{17} \mathrm{H}_{13} \mathrm{~N}_{2} \mathrm{OF}_{7}$ : C, 51.79; H, 3.32; N, 7.10; F, 33.73. Found : C, 51.87; H, 3.11; N, 6.97; F, 33.68.

3-Cyano-4-heptafluoroisopropyl-1-(p-methoxybenzyl)-1,4-dihydropyridine (3f'). Pale yellow oil.

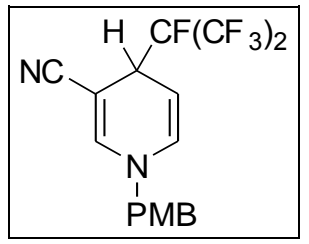

IR $\left(\mathrm{CCl}_{4}, v_{\max } / \mathrm{cm}^{-1}\right): 3009,2939,2842,2204,1687,1593,1515,1416,1294$, $1254,1223,1120,1034,977,748 .{ }^{1} \mathrm{H}$ NMR $(400 \mathrm{MHz}) \delta=3.82\left(3 \mathrm{H}, \mathrm{s}, \mathrm{OCH}_{3}\right)$, $4.30(1 \mathrm{H}, \mathrm{m}, \mathrm{CHCF}), 4.35\left(2 \mathrm{H}, \mathrm{s}, \mathrm{NCH}_{2}\right), 4.71(1 \mathrm{H}, \mathrm{m}, \mathrm{NCH}=\mathrm{CH}), 6.12(1 \mathrm{H}, \mathrm{d}$, $\left.{ }^{3} J_{\mathrm{HH}}=8.1 \mathrm{~Hz}, \mathrm{NCH}=\mathrm{CH}\right), 6.88\left(1 \mathrm{H}, \mathrm{d},{ }^{4} J_{\mathrm{HH}}=1.5 \mathrm{~Hz}, \mathrm{NCH}=\mathrm{CCN}\right), 6.91(2 \mathrm{H}, \mathrm{d}$, $\left.{ }^{3} J_{\mathrm{HH}}=8.7 \mathrm{~Hz}, \mathrm{PMB}\right), 7.11\left(2 \mathrm{H}, \mathrm{m},{ }^{3} J_{\mathrm{HH}}=8.8 \mathrm{~Hz}, \mathrm{PMB}\right) .{ }^{13} \mathrm{C} \mathrm{NMR}(100 \mathrm{MHz}) \delta=$ $37.5\left(\mathrm{~d},{ }^{2} J_{\mathrm{CF}}=21.6 \mathrm{~Hz}\right), 55.3,57.5,74.3\left(\mathrm{~d},{ }^{3} J_{\mathrm{CF}}=5.1 \mathrm{~Hz}\right), 91.1\left(\mathrm{dm},{ }^{1} J_{\mathrm{CF}}=206.9 \mathrm{~Hz},{ }^{2} J_{\mathrm{CF}}=28.4 \mathrm{~Hz}\right)$, $96.4\left(\mathrm{dd},{ }^{3} J_{\mathrm{CF}}=8.6 \mathrm{~Hz},{ }^{4} J_{\mathrm{CF}}=1.7 \mathrm{~Hz}\right), 114.5,120.0,120.7\left(\mathrm{qd},{ }^{1} J_{\mathrm{CF}}=288.8 \mathrm{~Hz},{ }^{2} J_{\mathrm{CF}}=28.4 \mathrm{~Hz}\right), 120.7$ $\left(\mathrm{qd},{ }^{1} J_{\mathrm{CF}}=287.1 \mathrm{~Hz},{ }^{2} J_{\mathrm{CF}}=26.7 \mathrm{~Hz}\right), 126.8,128.9,132.0,145.4,159.8 .{ }^{19} \mathrm{~F} \mathrm{NMR}(376 \mathrm{MHz}) \delta=$ $-179.80\left(1 \mathrm{~F}, \mathrm{md},{ }^{3} J=7.5 \mathrm{~Hz},{ }^{4} J_{\mathrm{FH}}=1.5 \mathrm{~Hz}, \mathrm{CF}\left(\mathrm{CF}_{3}\right)_{2}\right),-74.64\left(3 \mathrm{~F}, \mathrm{~m}, \mathrm{CF}(\mathrm{CF})_{2}\right),-72.61(3 \mathrm{~F}, \mathrm{~m}$, $\left.\mathrm{CF}\left(\mathrm{CF}_{3}\right)_{2}\right)$. MS (EI $\left.70 \mathrm{eV}\right) \mathrm{m} / z(\%): 394\left(\mathrm{M}^{+},<1\right), 225(10), 121(100)$. HRMS (EI) : calcd for $\mathrm{C}_{17} \mathrm{H}_{13} \mathrm{~N}_{2} \mathrm{OF}_{7}\left(\mathrm{M}^{+}\right)$394.0916, found 394.0911. Anal. Calcd for $\mathrm{C}_{17} \mathrm{H}_{13} \mathrm{~N}_{2} \mathrm{OF}_{7}$ : C, 51.79; H, 3.32; N, 7.10; F, 33.73. Found : C, 51.66; H, 3.07; N, 7.31; F, 33.62.

3-Benzoyl-6-heptafluoroisopropyl-1-(p-methoxybenzyl)-1,6-dihydropyridine (2g'). Yellow oil. IR

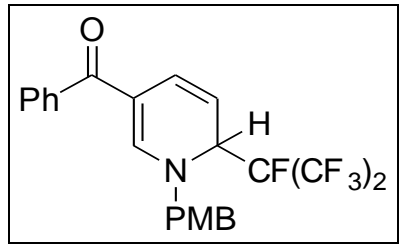

$\left(\mathrm{CH}_{2} \mathrm{Cl}_{2}, v_{\max } / \mathrm{cm}^{-1}\right): 2938,1614,1569,1514,1290,1254,1225,1109,716$. ${ }^{1} \mathrm{H}$ NMR (400 MHz) $\delta=3.80\left(3 \mathrm{H}, \mathrm{s}, \mathrm{OCH}_{3}\right), 4.48\left(2 \mathrm{H}, \mathrm{AB},{ }^{2} J_{\mathrm{HH}}=15.0\right.$, $\left.\mathrm{NCH}_{2}\right), 5.01-5.13(2 \mathrm{H}, \mathrm{m}, \mathrm{CHCHCF}), 6.88\left(2 \mathrm{H}, \mathrm{dm},{ }^{3} \mathrm{~J}_{\mathrm{HH}}=8.8 \mathrm{~Hz}, \mathrm{PMB}\right)$, 
$7.02\left(1 \mathrm{H}, \mathrm{dd},{ }^{3} J_{\mathrm{HH}}=8.9 \mathrm{~Hz},{ }^{4} J_{\mathrm{HH}}=1.1 \mathrm{~Hz}, \mathrm{CH}=\mathrm{CHCHCF}\right), 7.10\left(2 \mathrm{H}, \mathrm{dm},{ }^{3} J_{\mathrm{HH}}=8.7 \mathrm{~Hz}, \mathrm{PMB}\right), 7.20$ $\left(1 \mathrm{H}, \mathrm{d},{ }^{4} J_{\mathrm{HH}}=0.8 \mathrm{~Hz}, \mathrm{NCH}=\mathrm{CCOPh}\right), 7.39-7.44(2 \mathrm{H}, \mathrm{m}, \mathrm{Ph}), 7.45-7.49(1 \mathrm{H}, \mathrm{m}, \mathrm{Ph}), 7.54-7.57$ $(2 \mathrm{H}, \mathrm{m}, \mathrm{Ph}) .{ }^{13} \mathrm{C}$ NMR $(100 \mathrm{MHz}) \delta=55.3,58.4\left(\mathrm{~d},{ }^{2} J_{\mathrm{CF}}=19.8 \mathrm{~Hz}\right), 60.5\left(\mathrm{~d},{ }^{4} J_{\mathrm{CF}}=6.0 \mathrm{~Hz}\right), 92.0(\mathrm{~m})$, $104.8\left(\mathrm{dm},{ }^{3} J_{\mathrm{CF}}=5.2 \mathrm{~Hz}\right), 111.2,114.6,120.3\left(\mathrm{qd},{ }^{1} J_{\mathrm{CF}}=287.1 \mathrm{~Hz},{ }^{2} J_{\mathrm{CF}}=25.9 \mathrm{~Hz}\right), 120.7\left(\mathrm{qd},{ }^{1} J_{\mathrm{CF}}=\right.$ $\left.287.9 \mathrm{~Hz},{ }^{2} J_{\mathrm{CF}}=27.6 \mathrm{~Hz}\right), 126.1,127.5,128.2,128.7,129.2,130.9$, 139.2, 151.0, 159.9, 190.9. ${ }^{19} \mathrm{~F}$ $\operatorname{NMR}(376 \mathrm{MHz}) \delta=-177.39\left(1 \mathrm{~F}, \mathrm{~m}, \mathrm{CF}\left(\mathrm{CF}_{3}\right)_{2}\right),-73.85\left(3 \mathrm{~F}, \mathrm{~m}, \mathrm{CF}(\mathrm{CF})_{3}\right),-72.54\left(3 \mathrm{~F}, \mathrm{~m}, \mathrm{CF}\left(\mathrm{C} F_{3}\right)_{2}\right)$. MS (EI $70 \mathrm{eV}) \mathrm{m} / z(\%)$ : 473(M $\left.\mathrm{M}^{+}, 1\right), 304(22), 121(100)$. HRMS (EI) : calcd for $\mathrm{C}_{23} \mathrm{H}_{18} \mathrm{NO}_{2} \mathrm{~F}_{7}\left(\mathrm{M}^{+}\right)$ 473.1226, found 473.1204. Anal. Calcd for $\mathrm{C}_{23} \mathrm{H}_{18} \mathrm{NO}_{2} \mathrm{~F}_{7}: \mathrm{C}, 58.36 ; \mathrm{H}, 3.83 ; \mathrm{N}, 2.96 ; \mathrm{F}, 28.09$. Found : C, 58.41; H, 3.88; N, 2.82; F, 26.73.

3-Benzoyl-4-heptafluoroisopropyl-1-(p-methoxybenzyl)-1,4-dihydropyridine (3g'). Yellow oil. ${ }^{1} \mathrm{H}$

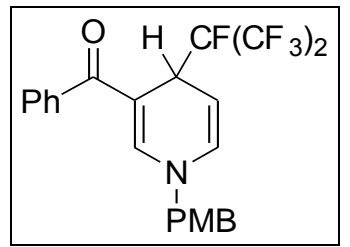
NMR $(400 \mathrm{MHz}) \delta=3.81\left(3 \mathrm{H}, \mathrm{s}, \mathrm{OCH}_{3}\right), 4.35\left(2 \mathrm{H}, \mathrm{s}, \mathrm{NCH}_{2}\right), 4.90\left(1 \mathrm{H}, \mathrm{t},{ }^{3} J=\right.$ $6.5 \mathrm{~Hz}, \mathrm{CHCF}), 5.18\left(1 \mathrm{H}, \mathrm{t},{ }^{3} J_{\mathrm{HH}}=5.5 \mathrm{~Hz}, \mathrm{NCH}=\mathrm{CH}\right), 6.16\left(1 \mathrm{H}, \mathrm{d},{ }^{3} J_{\mathrm{HH}}=7.8\right.$ $\mathrm{Hz}, \mathrm{NCH}=\mathrm{CH}), 6.88\left(2 \mathrm{H}, \mathrm{dm},{ }^{3} J_{\mathrm{HH}}=8.8 \mathrm{~Hz}, \mathrm{PMB}\right), 7.07\left(2 \mathrm{H}, \mathrm{dm},{ }^{3} J_{\mathrm{HH}}=8.8\right.$ $\mathrm{Hz}, \mathrm{PMB}), 7.37$ - 7.40 (2H, m, Ph), 7.48 (1H, s, NCH=CCOPh), $7.48-7.51$ $(1 \mathrm{H}, \mathrm{m}, \mathrm{Ph}), 7.57-7.61(2 \mathrm{H}, \mathrm{m}, \mathrm{Ph}) .{ }^{13} \mathrm{C} \mathrm{NMR}(100 \mathrm{MHz}) \delta=34.6\left(\mathrm{~d},{ }^{2} J_{\mathrm{CF}}=21.6 \mathrm{~Hz}\right), 57.6,65.8$, 92.0 (m), 99.0, 104.6, 114.4, c.a. 121 (m), 127.4, 128.3, 128.6, 128.7, 131.0, 131.9, 138.7, 145.9, 159.6, 194.7. ${ }^{19} \mathrm{~F}$ NMR $(376 \mathrm{MHz}) \delta=-183.32\left(1 \mathrm{~F}, \mathrm{~m}, \mathrm{CF}\left(\mathrm{CF}_{3}\right)_{2}\right),-75.36\left(3 \mathrm{~F}, \mathrm{~m}, \mathrm{CF}(\mathrm{CF})_{2}\right),-73.07(3 \mathrm{~F}, \mathrm{~m}$, $\left.\mathrm{CF}\left(\mathrm{CF}_{3}\right)_{2}\right)$.

3-Chloro-4-heptafluoroisopropyl-1-(p-methoxybenzyl)-1,4-dihydropyridine (3h'). Pale yellow oil,

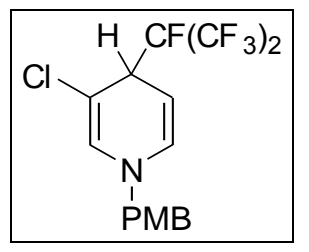
becomes dark-red after a few days at RT. IR (film, $v_{\max } / \mathrm{cm}^{-1}$ ) : 3075, 3007, 2938, 2840, 1725, 1676, 1613, 1587, 1514, 1415, 1399, 1304, 1280, 1251, 1218, 1175, $1115,1066,1037,976,953,813,745,538 .{ }^{1} \mathrm{H}$ NMR $(400 \mathrm{MHz}) \delta=3.81(3 \mathrm{H}, \mathrm{s}$, $\left.\mathrm{OCH}_{3}\right), 4.26\left(2 \mathrm{H}, \mathrm{s}, \mathrm{NCH}_{2}\right), 4.31\left(1 \mathrm{H}, \mathrm{ddm},{ }^{3} J_{\mathrm{HF}}=8.5 \mathrm{~Hz},{ }^{3} J_{\mathrm{HH}}=6.7 \mathrm{~Hz}, \mathrm{CHCF}\right)$, $4.41(1 \mathrm{H}, \mathrm{m}, \mathrm{NCH}=\mathrm{CH}), 6.12\left(1 \mathrm{H}, \mathrm{dt},{ }^{3} J_{\mathrm{HH}}=7.6 \mathrm{~Hz},{ }^{4} J_{\mathrm{HH}}=1.5 \mathrm{~Hz}, \mathrm{NCH}=\mathrm{CH}\right)$, $6.39\left(1 \mathrm{H}, \mathrm{d},{ }^{4} J_{\mathrm{HH}}=1.4 \mathrm{~Hz}, \mathrm{NCH}=\mathrm{CCl}\right), 6.89\left(2 \mathrm{H}, \mathrm{dm},{ }^{3} J_{\mathrm{HH}}=8.7 \mathrm{~Hz}, \mathrm{PMB}\right), 7.11\left(2 \mathrm{H}, \mathrm{dm},{ }^{3} J_{\mathrm{HH}}=8.8\right.$ $\mathrm{Hz}, \mathrm{PMB}) .{ }^{13} \mathrm{C}$ NMR $(100 \mathrm{MHz}) \delta=44.2\left(\mathrm{~d},{ }^{2} J_{\mathrm{CF}}=19.9 \mathrm{~Hz}\right), 55.3,56.7,90.9\left(\mathrm{~d},{ }^{3} J_{\mathrm{CF}}=7.7 \mathrm{~Hz}\right), 92.1$ $\left(\mathrm{dm},{ }^{1} J_{\mathrm{CF}}=210.3 \mathrm{~Hz},{ }^{2} J_{\mathrm{CF}}=28.5 \mathrm{~Hz}\right), 99.8,114.2,120.9\left(\mathrm{qd},{ }^{1} J_{\mathrm{CF}}=287.9 \mathrm{~Hz},{ }^{2} J_{\mathrm{CF}}=27.7 \mathrm{~Hz}\right), 128.5$, 128.7, 132.3, 133.0, 159.4. ${ }^{19} \mathrm{~F}$ NMR (376 MHz) $\delta=-176.10\left(1 \mathrm{~F}, \mathrm{~m}, \mathrm{CF}\left(\mathrm{CF}_{3}\right)_{2}\right),-74.07(3 \mathrm{~F}, \mathrm{~m}$, $\left.\mathrm{CF}\left(\mathrm{CF}_{3}\right)_{2}\right),-72.94\left(3 \mathrm{~F}, \mathrm{~m}, \mathrm{CF}\left(\mathrm{CF}_{3}\right)_{2}\right)$. HRMS (ESI, $\left.\mathrm{MeOH} / \mathrm{CH}_{2} \mathrm{Cl}_{2}\right)$ : calcd for $\mathrm{C}_{16} \mathrm{H}_{14} \mathrm{NOClF}_{7}\left(\mathrm{M}+\mathrm{H}^{+}\right)$ 
404.0647, found 404.0624. Anal. Calcd for $\mathrm{C}_{16} \mathrm{H}_{13} \mathrm{NOClF}_{7}: \mathrm{C}, 47.60 ; \mathrm{H}, 3.25 ; \mathrm{N}, 3.47 ; \mathrm{Cl}$, 8.78. Found : C, 47.45; H, 3.21; N, 3.46; Cl 8.69.

4-Ethoxy-2-heptafluoroisopropyl-1-(p-methoxybenzyl)-5-nitro-1,2-dihydropyridine (2i'). Pale

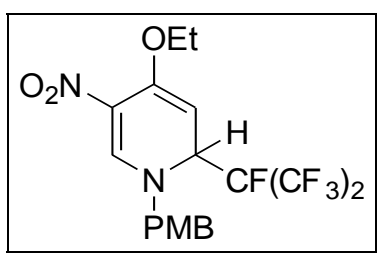

yellow solid, mp $100-102{ }^{\circ} \mathrm{C}$. IR $\left(\mathrm{KBr}, \nu_{\max } / \mathrm{cm}^{-1}\right): 3068,3001,2936,2838$, 1649, 1584, 1513, 1276, 1251, 1226, 1160, 1112, 1034, 983, 730. ${ }^{1} \mathrm{H}$ NMR $(400 \mathrm{MHz}) \delta=1.39\left(3 \mathrm{H}, \mathrm{t},{ }^{3} J_{\mathrm{HH}}=7.0 \mathrm{~Hz}, \mathrm{CH}_{2} \mathrm{CH}_{3}\right), 3.82\left(2 \mathrm{H}, \mathrm{q},{ }^{3} J_{\mathrm{HH}}=7.0\right.$ $\left.\mathrm{Hz}, \mathrm{CH}_{2} \mathrm{CH}_{3}\right), 3.83\left(3 \mathrm{H}, \mathrm{s}, \mathrm{OCH}_{3}\right), 3.98\left(1 \mathrm{H}, \mathrm{d},{ }^{3} \mathrm{~J}_{\mathrm{HH}}=5.6 \mathrm{~Hz}, \mathrm{CH}=\mathrm{C}(\mathrm{OEt})\right)$, $4.58\left(2 \mathrm{H}, \mathrm{AB},{ }^{2} J_{\mathrm{HH}}=15.4 \mathrm{~Hz}, \mathrm{NCH}_{2}\right), 5.06(1 \mathrm{H}, \mathrm{m}, \mathrm{CHCF}), 6.95(2 \mathrm{H}, \mathrm{dm}$, $\left.{ }^{3} J_{\mathrm{HH}}=8.7 \mathrm{~Hz}, \mathrm{PMB}\right), 7.20\left(2 \mathrm{H}, \mathrm{dm},{ }^{3} J_{\mathrm{HH}}=8.7 \mathrm{~Hz}, \mathrm{PMB}\right), 8.19\left(1 \mathrm{H}, \mathrm{d},{ }^{4} J_{\mathrm{HH}}=1.4 \mathrm{~Hz}, \mathrm{CH}=\mathrm{CNO}_{2}\right) .{ }^{13} \mathrm{C}$ $\operatorname{NMR}(100 \mathrm{MHz}) \delta=14.1,55.3,59.4\left(\mathrm{~d},{ }^{2} J_{\mathrm{CF}}=19.8 \mathrm{~Hz}\right), 60.6\left(\mathrm{~d},{ }^{4} J_{\mathrm{CF}}=6.9 \mathrm{~Hz}\right), 64.3,77.4\left(\mathrm{~d},{ }^{3} J_{\mathrm{CF}}=\right.$ $8.6 \mathrm{~Hz}), 91.9\left(\mathrm{dm},{ }^{1} J_{\mathrm{CF}}=208.6 \mathrm{~Hz},{ }^{2} J_{\mathrm{CF}}=28.5 \mathrm{~Hz}\right), 114.8,120.2\left(\mathrm{qd},{ }^{1} J_{\mathrm{CF}}=288.0 \mathrm{~Hz},{ }^{2} J_{\mathrm{CF}}=27.7 \mathrm{~Hz}\right)$, $120.5\left(\mathrm{qd},{ }^{1} J_{\mathrm{CF}}=287.0 \mathrm{~Hz},{ }^{2} J_{\mathrm{CF}}=23.8 \mathrm{~Hz}\right), 121.9,124.9,129.7,148.2,151.2,160.2 .{ }^{19} \mathrm{~F}$ NMR $(376$ $\mathrm{MHz}) \delta=-176.11\left(1 \mathrm{~F}, \mathrm{~m},{ }^{3} J=7.0 \mathrm{~Hz}, \mathrm{CF}\left(\mathrm{CF}_{3}\right)_{2}\right),-73.44\left(3 \mathrm{~F}, \mathrm{~m}, \mathrm{CF}(\mathrm{CF})_{2}\right),-72.13\left(3 \mathrm{~F}, \mathrm{~m}, \mathrm{CF}\left(\mathrm{C} F_{3}\right)_{2}\right)$. HRMS (ESI, $\mathrm{MeOH})$ : calcd for $\mathrm{C}_{18} \mathrm{H}_{17} \mathrm{~N}_{2} \mathrm{O}_{4} \mathrm{~F}_{7} \mathrm{Na}\left(\mathrm{M}+\mathrm{Na}^{+}\right)$481.0969, found 481.0964. Anal. Calcd for $\mathrm{C}_{18} \mathrm{H}_{17} \mathrm{~N}_{2} \mathrm{O}_{4} \mathrm{~F}_{7}$ : C, 47.17; H, 3.74; N, 6.11; F, 29.02. Found : C, 47.39; H, 3.76; N, 6.00; F, 28.35.

4-Cyano-2-heptafluoroisopropyl-1-(p-methoxybenzyl)-1,2-dihydropyridine (2j'). Yellow oil,

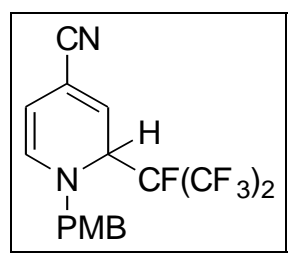

decomposes after a few days at RT. IR (film, $\left.v_{\max } / \mathrm{cm}^{-1}\right): 3074,3008,2959,2841$, 2232, 1613, 1563, 1514, 1295, 1252, 1228, 1177, 1139, 1035, 1013, 955, 831, 721.

${ }^{1} \mathrm{H}$ NMR $(200 \mathrm{MHz}) \delta=3.82\left(3 \mathrm{H}, \mathrm{s}, \mathrm{OCH}_{3}\right), 4.32\left(2 \mathrm{H}, \mathrm{s}, \mathrm{NCH}_{2}\right), 5.04(1 \mathrm{H}, \mathrm{dd}$, $\left.{ }^{3} J_{\mathrm{HH}}=7.1 \mathrm{~Hz},{ }^{4} J_{\mathrm{HH}}=1.6 \mathrm{~Hz}, \mathrm{NCH}=\mathrm{CH}\right), 5.17\left(1 \mathrm{H}, \mathrm{ddm},{ }^{3} J_{\mathrm{HF}}=8.5 \mathrm{~Hz},{ }^{3} J_{\mathrm{HH}}=6.6\right.$ $\left.\mathrm{Hz},{ }^{4} J_{\mathrm{HH}}=1.4 \mathrm{~Hz}, \mathrm{CHCF}\right), 5.38\left(1 \mathrm{H}, \mathrm{d},{ }^{3} J_{\mathrm{HH}}=6.5 \mathrm{~Hz}, \mathrm{CH}=\mathrm{CCN}\right), 6.37(1 \mathrm{H}, \mathrm{dt}$, $\left.{ }^{3} J_{\mathrm{HH}}=7.1 \mathrm{~Hz},{ }^{4} J_{\mathrm{HH}}=1.1 \mathrm{~Hz}, \mathrm{NCH}=\mathrm{CH}\right), 6.91\left(2 \mathrm{H}, \mathrm{d},{ }^{3} J_{\mathrm{HH}}=8.7 \mathrm{~Hz}, \mathrm{PMB}\right), 7.15\left(2 \mathrm{H}, \mathrm{m},{ }^{3} J_{\mathrm{HH}}=8.9 \mathrm{~Hz}\right.$, PMB). ${ }^{13} \mathrm{C}$ NMR (50 MHz) $\delta=55.3,58.4\left(\mathrm{~d},{ }^{2} J_{\mathrm{CF}}=21.2 \mathrm{~Hz}\right), 59.3\left(\mathrm{~d},{ }^{4} J_{\mathrm{CF}}=4.8 \mathrm{~Hz}\right)$, c.a. $90(\mathrm{~m}), 95.5$, $113.3\left(\mathrm{~d},{ }^{3} J_{\mathrm{CF}}=7.7 \mathrm{~Hz}\right), 114.5,115.8,117.0$, c.a. $120.5\left(\mathrm{qm},{ }^{1} J_{\mathrm{CF}}=\right.$ c.a. $\left.280 \mathrm{~Hz}\right), 127.4,129.0,139.2$, 159.7. ${ }^{19} \mathrm{~F}$ NMR $(376 \mathrm{MHz}) \delta=-180.87\left(1 \mathrm{~F}, \mathrm{~m}, \mathrm{CF}\left(\mathrm{CF}_{3}\right)_{2}\right),-73.86\left(3 \mathrm{~F}, \mathrm{~m}, J_{\mathrm{FF}}=8.9 \mathrm{~Hz}, \mathrm{CF}(\mathrm{CF})_{2}\right)$, -73.14 (3F, m, $\left.J_{\mathrm{FF}}=8.9 \mathrm{~Hz}, \mathrm{CF}\left(\mathrm{CF}_{3}\right)_{2}\right)$. HRMS (ESI) : calcd for $\mathrm{C}_{17} \mathrm{H}_{13} \mathrm{~N}_{2} \mathrm{OF}_{7} \mathrm{Na}\left(\mathrm{M}+\mathrm{Na}^{+}\right) 417.0808$, found 417.0829. Anal. Calcd for $\mathrm{C}_{17} \mathrm{H}_{13} \mathrm{~N}_{2} \mathrm{OF}_{7}$ : C, 51.79; H, 3.32; N, 7.10; F, 33.73. Found : C, 51.53; H, 3.38; N, 6.90; F, 33.81. 
2-Heptafluoroisopropyl-5-methoxy-1-(p-methoxybenzyl)-1,2-dihydroquinoline (2k'). Pale yellow

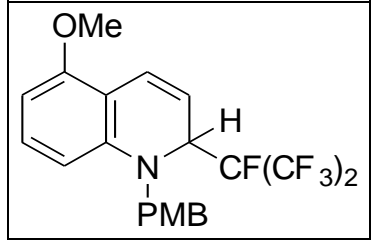
oil. IR (film, $v_{\max } / \mathrm{cm}^{-1}$ ) : 3071, 3005, 2957, 2839, 1578, 1513, 1478, 1294, $1251,1221,1155,1108,1037,974,751 .{ }^{1} \mathrm{H}$ NMR $(400 \mathrm{MHz}) \delta=3.75(3 \mathrm{H}$, $\left.\mathrm{s}, \mathrm{OCH}_{3}\right), 3.79\left(3 \mathrm{H}, \mathrm{s}, \mathrm{OCH}_{3}\right), 4.36\left(1 \mathrm{H}, \mathrm{d},{ }^{2} J_{\mathrm{HH}}=15.8 \mathrm{~Hz}, \mathrm{NCH}_{2}\right), 4.91(1 \mathrm{H}$, $\left.\mathrm{d},{ }^{2} J_{\mathrm{HH}}=15.8 \mathrm{~Hz}, \mathrm{NCH}_{2}\right), 5.00-5.06(1 \mathrm{H}, \mathrm{m}, \mathrm{CHCF}), 5.45-5.51(1 \mathrm{H}, \mathrm{m}$, $\mathrm{CHCHCF}), 6.28\left(1 \mathrm{H}, \mathrm{dd},{ }^{3} J_{\mathrm{HH}}=8.3 \mathrm{~Hz},{ }^{4} J_{\mathrm{HH}}=0.5 \mathrm{~Hz}, \mathrm{H}_{\mathrm{arom}}\right), 6.37\left(1 \mathrm{H}, \mathrm{dd},{ }^{3} J_{\mathrm{HH}}=8.4 \mathrm{~Hz},{ }^{4} J_{\mathrm{HH}}=0.5\right.$ $\mathrm{Hz}, \mathrm{Hz}, \mathrm{CH}=\mathrm{CHCHCF}), 6.80\left(2 \mathrm{H}, \mathrm{dm},{ }^{3} J_{\mathrm{HH}}=8.8 \mathrm{~Hz}, \mathrm{PMB}\right), 7.02\left(1 \mathrm{H}, \mathrm{t},{ }^{3} J_{\mathrm{HH}}=8.3 \mathrm{~Hz}, \mathrm{H}_{\text {arom }}\right), 7.07-$ $7.14\left(1 \mathrm{H}, \mathrm{m}, \mathrm{H}_{\text {arom }}\right), 7.07-7.14(2 \mathrm{H}, \mathrm{m}, \mathrm{PMB}) .{ }^{13} \mathrm{C} \mathrm{NMR}(100 \mathrm{MHz}) \delta=55.1,55.4,55.7\left(\mathrm{~d},{ }^{4} J_{\mathrm{CF}}=4.3\right.$ $\mathrm{Hz}), 59.3\left(\mathrm{~d},{ }^{2} J_{\mathrm{CF}}=19.8 \mathrm{~Hz}\right), 92.1\left(\mathrm{dm},{ }^{1} J_{\mathrm{CF}}=205.2 \mathrm{~Hz},{ }^{2} J_{\mathrm{CF}}=29.3 \mathrm{~Hz}\right), 100.8,107.9,111.2,112.1(\mathrm{~d}$, $\left.{ }^{3} J_{\mathrm{CF}}=6.9 \mathrm{~Hz}\right), 114.1,120.7\left(\mathrm{qd},{ }^{1} J_{\mathrm{CF}}=287.1 \mathrm{~Hz},{ }^{2} J_{\mathrm{CF}}=28.4 \mathrm{~Hz}\right), 120.8\left(\mathrm{qd},{ }^{1} J_{\mathrm{CF}}=288.0 \mathrm{~Hz},{ }^{2} J_{\mathrm{CF}}=25.9\right.$ $\mathrm{Hz}), 125.0,128.5,128.9,129.5,144.0,155.4,158.9 .{ }^{19} \mathrm{~F}$ NMR $(376 \mathrm{MHz}) \delta=-180.05(1 \mathrm{~F}, \mathrm{~m}$, $\left.\mathrm{C} F\left(\mathrm{CF}_{3}\right)_{2}\right),-74.09\left(3 \mathrm{~F}, \mathrm{~m},{ }^{3} J_{\mathrm{FF}}=8.7 \mathrm{~Hz}, \mathrm{CF}\left(\mathrm{CF}_{3}\right)_{2}\right),-72.85\left(3 \mathrm{~F}, \mathrm{~m},{ }^{3} J_{\mathrm{FF}}=8.7 \mathrm{~Hz}, \mathrm{CF}\left(\mathrm{CF}_{3}\right)_{2}\right)$. MS $(\mathrm{EI} 70$ eV) $m / z(\%): 448(<1), 280(20), 159(4), 121(100)$. HRMS (EI) : calcd for $\mathrm{C}_{21} \mathrm{H}_{17} \mathrm{NO}_{2} \mathrm{~F}_{7}\left([\mathrm{M}-\mathrm{H}]^{+}\right)$ 448.1148, found 448.1128. Anal. Calcd for $\mathrm{C}_{21} \mathrm{H}_{18} \mathrm{NO}_{2} \mathrm{~F}_{7}: \mathrm{C}, 56.13 ; \mathrm{H}, 4.04 ; \mathrm{N}, 3.12 ; \mathrm{F}, 29.59$. Found : C, 56.00; H, 4.06; N, 3.09; F, 28.97.

4-Heptafluoroisopropyl-5-methoxy-1-(p-methoxybenzyl)-1,4-dihydroquinoline (3k'). Pale yellow

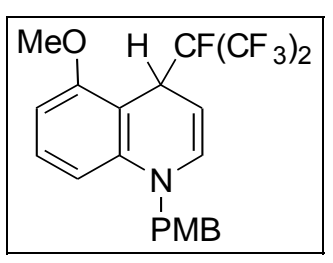
oil. IR $\left(\mathrm{CH}_{2} \mathrm{Cl}_{2}, v_{\max } / \mathrm{cm}^{-1}\right): 2939,2840,1665,1599,1514,1479,1388,1283$, $1219,1175,1128,977,791,732 .{ }^{1} \mathrm{H} \operatorname{NMR}(400 \mathrm{MHz}) \delta=3.78\left(3 \mathrm{H}, \mathrm{s}, \mathrm{OCH}_{3}\right)$, $3.79\left(3 \mathrm{H}, \mathrm{s}, \mathrm{OCH}_{3}\right), 4.58\left(1 \mathrm{H}, \mathrm{t},{ }^{3} J_{\mathrm{HH}}=6.6 \mathrm{~Hz}, \mathrm{NCH}=\mathrm{CH}\right), 4.66\left(2 \mathrm{H}, \mathrm{s}, \mathrm{NCH}_{2}\right)$, $5.13(1 \mathrm{H}, \mathrm{m}, \mathrm{CHCF}), 6.38\left(2 \mathrm{H}, \mathrm{d},{ }^{3} \mathrm{~J}_{\mathrm{HH}}=7.7 \mathrm{~Hz}, \mathrm{NCH}=\mathrm{CH}, \mathrm{H}_{\text {arom }}\right), 6.51(1 \mathrm{H}, \mathrm{dd}$, $\left.{ }^{3} J_{\mathrm{HH}}=8.1 \mathrm{~Hz},{ }^{4} J_{\mathrm{HH}}=0.6 \mathrm{~Hz}, \mathrm{H}_{\mathrm{arom}}\right), 6.85\left(2 \mathrm{H}, \mathrm{dm},{ }^{3} J_{\mathrm{HH}}=8.8 \mathrm{~Hz}, \mathrm{PMB}\right), 7.09\left(1 \mathrm{H}, \mathrm{t},{ }^{3} J_{\mathrm{HH}}=8.3 \mathrm{~Hz}\right.$, $\left.\mathrm{H}_{\text {arom }}\right), 7.13\left(2 \mathrm{H}, \mathrm{dm},{ }^{3} J_{\mathrm{HH}}=8.8 \mathrm{~Hz}, \mathrm{PMB}\right) .{ }^{13} \mathrm{C} \mathrm{NMR}(100 \mathrm{MHz}) \delta=33.8\left(\mathrm{~d},{ }^{2} J_{\mathrm{CF}}=21.6 \mathrm{~Hz}\right), 53.9$, $55.2,55.5,90.6\left(\mathrm{~d},{ }^{3} J_{\mathrm{CF}}=5.2 \mathrm{~Hz}\right), 91.5\left(\mathrm{dm},{ }^{1} J_{\mathrm{CF}}=212.1 \mathrm{~Hz},{ }^{2} J_{\mathrm{CF}}=28.4 \mathrm{~Hz}\right), 103.1,103.4,105.5$, $114.1,121.1\left(\mathrm{qd},{ }^{1} J_{\mathrm{CF}}=287.9 \mathrm{~Hz},{ }^{2} J_{\mathrm{CF}}=29.3 \mathrm{~Hz}\right), 121.6\left(\mathrm{qd},{ }^{1} J_{\mathrm{CF}}=287.1 \mathrm{~Hz},{ }^{2} J_{\mathrm{CF}}=28.4 \mathrm{~Hz}\right), 127.7$, 128.7, 129.2, 136.2, 143.0, 158.8, 158.9. ${ }^{19} \mathrm{~F}$ NMR $(376 \mathrm{MHz}) \delta=-180.92\left(1 \mathrm{~F}, \mathrm{~m},{ }^{3} J=5.6 \mathrm{~Hz}\right.$, $\left.\mathrm{CF}\left(\mathrm{CF}_{3}\right)_{2}\right),-75.76\left(3 \mathrm{~F}, \mathrm{~m},{ }^{3} J_{\mathrm{FF}}=8.3 \mathrm{~Hz}, \mathrm{CF}\left(\mathrm{CF}_{3}\right)_{2}\right),-72.92\left(3 \mathrm{~F}, \mathrm{~m},{ }^{3} J_{\mathrm{FF}}=8.3 \mathrm{~Hz}, \mathrm{CF}\left(\mathrm{C} F_{3}\right)_{2}\right)$. MS (EI 70 eV) $m / z(\%): 449\left(\mathrm{M}^{+},<1\right), 280(25), 159(4), 121(100)$. HRMS (EI) : calcd for $\mathrm{C}_{21} \mathrm{H}_{18} \mathrm{NO}_{2} \mathrm{~F}_{7}\left(\mathrm{M}^{+}\right)$ 449.1226, found 449.1216. Anal. Calcd for $\mathrm{C}_{21} \mathrm{H}_{18} \mathrm{NO}_{2} \mathrm{~F}_{7}: \mathrm{C}, 56.13 ; \mathrm{H}, 4.04 ; \mathrm{N}, 3.12 ; \mathrm{F}, 29.59$. Found : C, 56.07; H, 4.12; N, 3.06; F, 29.55. 
3-Heptafluoroisopropyl-4-(p-methoxybenzyl)-3,4-dihydrophenantroline (2l'). Pale yellow oil. IR

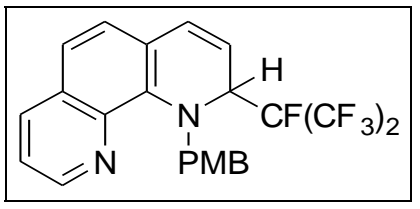

(film, $v_{\max } / \mathrm{cm}^{-1}$ ) : 3055, 3005, 2955, 2838, 1611, 1512, 1466, 1369, 1300, $1245,1223,1175,1108,1035,998,831,734,698 .{ }^{1} \mathrm{H}$ NMR $(400 \mathrm{MHz}) \delta$ $=3.72\left(3 \mathrm{H}, \mathrm{s}, \mathrm{OCH}_{3}\right), 4.48\left(1 \mathrm{H}, \mathrm{d},{ }^{2} J_{\mathrm{HH}}=15.5 \mathrm{~Hz}, \mathrm{NCH}_{2}\right), 5.04(1 \mathrm{H}, \mathrm{dd}$, $\left.{ }^{3} J_{\mathrm{HF}}=14.8 \mathrm{~Hz},{ }^{3} J_{\mathrm{HH}}=6.0 \mathrm{~Hz}, \mathrm{CHCF}\right), 5.53(1 \mathrm{H}, \mathrm{m}, \mathrm{CHCHCF}), 5.99(1 \mathrm{H}$, $\left.\mathrm{d},{ }^{2} J_{\mathrm{HH}}=15.4 \mathrm{~Hz}, \mathrm{NCH}_{2}\right), 6.70-6.72\left(1 \mathrm{H}, \mathrm{d},{ }^{3} J_{\mathrm{HF}}=8.7 \mathrm{~Hz}, \mathrm{CH}=\mathrm{CHCHCF}\right), 6.72\left(2 \mathrm{H}, \mathrm{dm},{ }^{3} J_{\mathrm{HH}}=8.7\right.$ $\mathrm{Hz}, \mathrm{PMB}), 7.06\left(2 \mathrm{H}, \mathrm{dm},{ }^{3} J_{\mathrm{HH}}=8.7 \mathrm{~Hz}, \mathrm{PMB}\right), 7.18\left(1 \mathrm{H}, \mathrm{d},{ }^{3} J_{\mathrm{HH}}=8.2 \mathrm{~Hz}, \mathrm{H}_{\text {arom }}\right), 7.29\left(1 \mathrm{H}, \mathrm{dd},{ }^{3} J_{\mathrm{HH}}=\right.$ $\left.8.2 \mathrm{~Hz},{ }^{3} J_{\mathrm{HH}}=4.1 \mathrm{~Hz}, \mathrm{H}_{\text {arom }}\right), 7.30\left(1 \mathrm{H}, \mathrm{d},{ }^{3} J_{\mathrm{HH}}=8.2 \mathrm{~Hz}, \mathrm{H}_{\text {arom }}\right), 7.99\left(1 \mathrm{H}, \mathrm{dd},{ }^{3} J_{\mathrm{HH}}=8.4 \mathrm{~Hz},{ }^{4} J_{\mathrm{HH}}=1.8\right.$ $\left.\mathrm{Hz}, \mathrm{H}_{\text {arom }}\right), 8.86\left(1 \mathrm{H}, \mathrm{dd},{ }^{3} J_{\mathrm{HH}}=4.1 \mathrm{~Hz},{ }^{4} J_{\mathrm{HH}}=1.8 \mathrm{~Hz}, \mathrm{H}_{\text {arom }}\right) \cdot{ }^{13} \mathrm{C} \mathrm{NMR}(100 \mathrm{MHz}) \delta=55.1,59.2(\mathrm{~d}$, $\left.{ }^{2} J_{\mathrm{CF}}=21.6 \mathrm{~Hz}\right), 61.2,91.7\left(\mathrm{dm},{ }^{1} J_{\mathrm{CF}}=207.8 \mathrm{~Hz},{ }^{2} J_{\mathrm{CF}}=29.3 \mathrm{~Hz}\right), 113.6,116.1,120.5,120.8,120.8(\mathrm{qd}$, $\left.{ }^{1} J_{\mathrm{CF}}=289.7 \mathrm{~Hz},{ }^{2} J_{\mathrm{CF}}=27.6 \mathrm{~Hz}\right), 120.9\left(\mathrm{qd},{ }^{1} J_{\mathrm{CF}}=286.2 \mathrm{~Hz},{ }^{2} J_{\mathrm{CF}}=26.7 \mathrm{~Hz}\right), 124.3,125.8,129.4,129.8$, $130.4,131.4,136.2,140.5,141.8,147.9,158.9 .{ }^{19} \mathrm{~F}$ NMR $(376 \mathrm{MHz}) \delta=-185.20\left(1 \mathrm{~F}, \mathrm{~m},{ }^{3} J=7.6 \mathrm{~Hz}\right.$, $\left.\mathrm{CF}\left(\mathrm{CF}_{3}\right)_{2}\right),-73.42\left(3 \mathrm{~F}, \mathrm{~m},{ }^{3} J_{\mathrm{FF}}=8.3 \mathrm{~Hz}, \mathrm{CF}(\mathrm{CF})_{3}\right),-73.06\left(3 \mathrm{~F}, \mathrm{~m},{ }^{3} J_{\mathrm{FF}}=8.3 \mathrm{~Hz}, \mathrm{CF}\left(\mathrm{C} F_{3}\right)_{2}\right)$. HRMS (ESI) : calcd for $\mathrm{C}_{23} \mathrm{H}_{18} \mathrm{~N}_{2} \mathrm{OF}_{7}\left(\mathrm{M}+\mathrm{H}^{+}\right)$471.1302, found 471.1298. Anal. Calcd for $\mathrm{C}_{23} \mathrm{H}_{17} \mathrm{~N}_{2} \mathrm{OF}_{7}$ : C, 58.73; H, 3.64; N, 5.96; F, 28.27. Found : C, 58.74; H, 3.65; N, 6.02; F, 28.22.

1-Heptafluoroisopropyl-4-(p-methoxybenzyl)-1,4-dihydrophenantroline (3I'). Pale yellow oil. IR

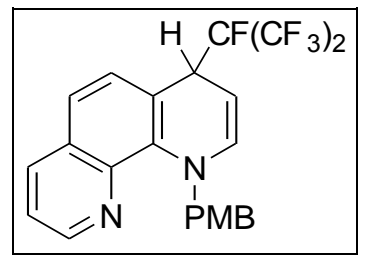

$\left(\mathrm{CH}_{2} \mathrm{Cl}_{2}, v_{\max } / \mathrm{cm}^{-1}\right): 3064,3005,2955,2907,2837,1668,1612,1512,1459$, 1287, 1248, 1223, 1173, 1122, 963, 830, 694. ${ }^{1} \mathrm{H}$ NMR $(400 \mathrm{MHz}) \delta=3.79$ $\left(3 \mathrm{H}, \mathrm{s}, \mathrm{OCH}_{3}\right), 4.74(2 \mathrm{H}, \mathrm{m}, \mathrm{CHCF}, \mathrm{NCH}=\mathrm{CH}), 4.85\left(1 \mathrm{H}, \mathrm{d},{ }^{2} J_{\mathrm{HH}}=14.6 \mathrm{~Hz}\right.$, $\left.\mathrm{NCH}_{2}\right), 6.33\left(1 \mathrm{H}, \mathrm{d},{ }^{2} J_{\mathrm{HH}}=14.4 \mathrm{~Hz}, \mathrm{NCH}_{2}\right), 6.35\left(1 \mathrm{H}, \mathrm{d},{ }^{3} J_{\mathrm{HH}}=5.8 \mathrm{~Hz}\right.$, $\mathrm{NCH}=\mathrm{CH}), 6.86\left(2 \mathrm{H}, \mathrm{dm},{ }^{3} \mathrm{~J}_{\mathrm{HH}}=8.8 \mathrm{~Hz}, \mathrm{PMB}\right), 7.23\left(1 \mathrm{H}, \mathrm{m}, \mathrm{H}_{\text {arom }}\right), 7.34(1 \mathrm{H}$, $\left.\mathrm{dd},{ }^{3} J_{\mathrm{HH}}=8.2 \mathrm{~Hz}, 4.1 \mathrm{~Hz}, \mathrm{H}_{\mathrm{arom}}\right), 7.37-7.44\left(3 \mathrm{H}, \mathrm{m}, \mathrm{PMB}, \mathrm{H}_{\text {arom }}\right), 8.07\left(1 \mathrm{H}, \mathrm{dd},{ }^{3} J_{\mathrm{HH}}=8.4 \mathrm{~Hz},{ }^{4} J_{\mathrm{HH}}=\right.$ $\left.1.8 \mathrm{~Hz}, \mathrm{H}_{\text {arom }}\right), 8.72\left(1 \mathrm{H}, \mathrm{dd},{ }^{3} J_{\mathrm{HH}}=4.0 \mathrm{~Hz},{ }^{4} J_{\mathrm{HH}}=1.8 \mathrm{~Hz}, \mathrm{H}_{\text {arom }}\right) .{ }^{13} \mathrm{C} \mathrm{NMR}(100 \mathrm{MHz}) \delta=42.0\left(\mathrm{~d},{ }^{2} J_{\mathrm{CF}}\right.$ $=19.8 \mathrm{~Hz}), 55.2,55.9,91.6\left(\mathrm{dm},{ }^{1} J_{\mathrm{CF}}=206.9 \mathrm{~Hz},{ }^{2} J_{\mathrm{CF}}=28.4 \mathrm{~Hz}\right), 93.3\left(\mathrm{~d},{ }^{3} J_{\mathrm{CF}}=6.9 \mathrm{~Hz}\right), 113.6,115.4$, $120.9,120.9\left(\mathrm{qd},{ }^{1} J_{\mathrm{CF}}=287.9 \mathrm{~Hz},{ }^{2} J_{\mathrm{CF}}=28.4 \mathrm{~Hz}\right), 121.0,121.3\left(\mathrm{qd},{ }^{1} J_{\mathrm{CF}}=287.9 \mathrm{~Hz},{ }^{2} J_{\mathrm{CF}}=27.6 \mathrm{~Hz}\right)$, $129.2,129.5,130.1\left(\mathrm{~d},{ }^{3} J_{\mathrm{CF}}=4.3 \mathrm{~Hz}\right), 131.2,136.6,137.4,139.1,140.5,146.9,158.7 .{ }^{19} \mathrm{~F}$ NMR $(376$ $\mathrm{MHz}) \delta=-175.53\left(1 \mathrm{~F}, \mathrm{~m}, \mathrm{CF}\left(\mathrm{CF}_{3}\right)_{2}\right),-74.00\left(3 \mathrm{~F}, \mathrm{~m},{ }^{3} J_{\mathrm{FF}}=8.3 \mathrm{~Hz}, \mathrm{CF}(\mathrm{CF})_{2}\right),-71.95\left(3 \mathrm{~F}, \mathrm{~m},{ }^{3} J_{\mathrm{FF}}=8.3\right.$ $\left.\mathrm{Hz}, \mathrm{CF}\left(\mathrm{CF}_{3}\right)_{2}\right)$. MS (EI $\left.70 \mathrm{eV}\right) \mathrm{m} / z(\%): 470\left(\mathrm{M}^{+}, 1\right), 301(25), 121(100)$. HRMS (EI) : calcd for $\mathrm{C}_{23} \mathrm{H}_{17} \mathrm{~N}_{2} \mathrm{OF}_{7}\left(\mathrm{M}^{+}\right)$470.1229, found 470.1235. Anal. Calcd for $\mathrm{C}_{23} \mathrm{H}_{17} \mathrm{~N}_{2} \mathrm{OF}_{7}$ : C, 58.73; $\mathrm{H}, 3.64 ; \mathrm{N}$, 
5.96; F, 28.27. Found : C, 58.63; H, 3.62; N, 5.85; F, 27.44.

4-Bromo-1-heptafluoroisopropyl-2-(p-methoxybenzyl)-1,2-dihydroisoquinoline (2m'). Pale yellow

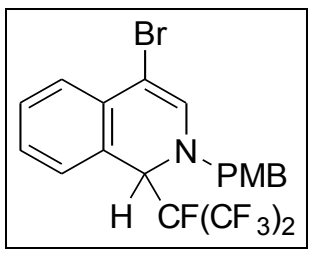
oil. IR (film, $\nu_{\max } / \mathrm{cm}^{-1}$ ) : 3067, 3003, 2958, 2937, 2838, 1614, 1513, 1287, 1228, 1174, 1113, 1035, 946, 760. ${ }^{1} \mathrm{H}$ NMR (400 MHz) $\delta=3.75\left(3 \mathrm{H}, \mathrm{s}, \mathrm{OCH}_{3}\right), 4.35$ $\left(2 \mathrm{H}, \mathrm{AB},{ }^{2} J_{\mathrm{HH}}=15.1 \mathrm{~Hz}, \mathrm{NCH}_{2}\right), 5.43\left(1 \mathrm{H}, \mathrm{d},{ }^{3} J_{\mathrm{HF}}=7.6 \mathrm{~Hz}, \mathrm{CHCF}\right), 6.54(1 \mathrm{H}, \mathrm{d}$, $\left.{ }^{4} J_{\mathrm{HH}}=1.2 \mathrm{~Hz}, \mathrm{CH}=\mathrm{CBr}\right), 6.78\left(2 \mathrm{H}, \mathrm{dm},{ }^{3} J_{\mathrm{HH}}=8.8 \mathrm{~Hz}, \mathrm{PMB}\right), 6.93\left(1 \mathrm{H}, \mathrm{d},{ }^{3} J_{\mathrm{HH}}=\right.$ $\left.7.7 \mathrm{~Hz}, \mathrm{H}_{\text {arom }}\right), 7.02\left(2 \mathrm{H}, \mathrm{dm},{ }^{3} J_{\mathrm{HH}}=8.8 \mathrm{~Hz}, \mathrm{PMB}\right), 7.16\left(1 \mathrm{H}, \mathrm{td},{ }^{3} J_{\mathrm{HH}}=7.6 \mathrm{~Hz}\right.$, $\left.{ }^{4} J_{\mathrm{HH}}=1.4 \mathrm{~Hz}, \mathrm{H}_{\text {arom }}\right), 7.34\left(1 \mathrm{H}, \mathrm{td},{ }^{3} J_{\mathrm{HH}}=7.6 \mathrm{~Hz},{ }^{4} J_{\mathrm{HH}}=1.2 \mathrm{~Hz}, \mathrm{H}_{\text {arom }}\right), 7.44\left(1 \mathrm{H}, \mathrm{dd},{ }^{3} J_{\mathrm{HH}}=7.8 \mathrm{~Hz},{ }^{4} J_{\mathrm{HH}}\right.$ $\left.=1.2 \mathrm{~Hz}, \mathrm{H}_{\text {arom }}\right){ }^{13} \mathrm{C} \mathrm{NMR}(100 \mathrm{MHz}) \delta=55.2,58.9\left(\mathrm{~d},{ }^{4} J_{\mathrm{CF}}=1.7 \mathrm{~Hz}\right), 61.2\left(\mathrm{~d},{ }^{2} J_{\mathrm{CF}}=22.4 \mathrm{~Hz}\right), 90.5$ $\left(\mathrm{dm},{ }^{1} J_{\mathrm{CF}}=206.0 \mathrm{~Hz},{ }^{2} J_{\mathrm{CF}}=30.2 \mathrm{~Hz}\right), 95.9,114.2,119.7\left(\mathrm{~d},{ }^{3} J_{\mathrm{CF}}=2.6 \mathrm{~Hz}\right), 120.7\left(\mathrm{qd},{ }^{1} J_{\mathrm{CF}}=288.8 \mathrm{~Hz}\right.$, $\left.{ }^{2} J_{\mathrm{CF}}=25.9 \mathrm{~Hz}\right), 120.9\left(\mathrm{qd},{ }^{1} J_{\mathrm{CF}}=285.4 \mathrm{~Hz},{ }^{2} J_{\mathrm{CF}}=27.6 \mathrm{~Hz}\right), 123.3,126.9,128.0,128.4,128.7,129.4$, 132.5, 135.6, 159.4. ${ }^{19} \mathrm{~F}$ NMR (376 MHz) $\delta=-179.98\left(1 \mathrm{~F}, \mathrm{~m},{ }^{3} J=8.3 \mathrm{~Hz}, \mathrm{CF}\left(\mathrm{CF}_{3}\right)_{2}\right),-72.47(3 \mathrm{~F}, \mathrm{~m}$, $\left.{ }^{3} J_{\mathrm{FF}}=9.0 \mathrm{~Hz}, \mathrm{CF}\left(\mathrm{CF}_{3}\right)_{2}\right),-72.17\left(3 \mathrm{~F}, \mathrm{~m},{ }^{3} J_{\mathrm{FF}}=8.3 \mathrm{~Hz}, \mathrm{CF}\left(\mathrm{CF}_{3}\right)_{2}\right)$. HRMS (ESI, MeOH) : calcd for $\mathrm{C}_{20} \mathrm{H}_{16} \mathrm{NO}^{79} \mathrm{BrF}_{7}\left(\mathrm{M}+\mathrm{H}^{+}\right)$498.0298, found 498.0308. Anal. Calcd for $\mathrm{C}_{20} \mathrm{H}_{15} \mathrm{NOBrF}_{7}$ : C, 48.21; $\mathrm{H}, 3.03$; N, 2.81; Br, 16.04; F, 26.69. Found : C, 47.96; H, 2.80; N, 2.79; Br, 16.23; F, 25.27.

General procedure for synthesis of $2 n^{\prime}, 20^{\prime}$ and 3o' from weakly nucleophilic pyridines 6 or 7 (with quarternization of nitrogen promoted by silver salts; scheme 9 in the main text). To a flamedried round-bottomed flask containing dry $\mathrm{MeCN}(3.0 \mathrm{~mL})$ the appropriate pyridine derivative $(0.95$ mmol) and $\mathrm{PMBBr}(201 \mathrm{mg}, 143 \mu \mathrm{L}, 1.00 \mathrm{mmol})$ were added under argon atmosphere. The reaction mixure was cooled to $-75^{\circ} \mathrm{C}$ and silver tetrafluoroborate $(195 \mathrm{mg}, 1.00 \mathrm{mmol})$ was added in one portion with vigorous stirring. After the reaction mixture had reached room temperature, stirring was switched off so that the $\mathrm{AgBr}$ precipitate could accumulate at the bottom of the flask. In the meantime HFP (c.a. $0.6 \mathrm{~g}, 4 \mathrm{mmol}$ ) was condensed in a glass pressure tube (c.a. $3.5 \mathrm{~mL}$ volume) at $-75^{\circ} \mathrm{C}$ under argon atmosphere. The solution from above the precipitate of $\mathrm{AgBr}$ was then transferred into the pressure tube. Spray-dried $\mathrm{KF}_{(\mathrm{s})}(276 \mathrm{mg}, 4.75 \mathrm{mmol})$ was then added and the pressure tube was closed with a teflon valve. The reaction was performed at room temperature for $3 \mathrm{~h}$ with vigorous stirring. Isolation of products was carried out in the way described above for the reactions of other $N$ alkylazinium salts. 


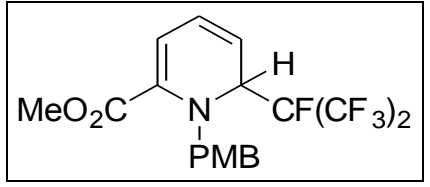

Yellow oil, quickly decomposes at RT. IR (film, $\left.v_{\max } / \mathrm{cm}^{-1}\right): 3004,2956$, $2840,1725,1613,1514,1464,1297,1251,1223,1156,1035,742 .{ }^{1} \mathrm{H}$ NMR $(400 \mathrm{MHz}) \delta=3.76\left(3 \mathrm{H}, \mathrm{s}, \mathrm{OCH}_{3}\right), 3.78\left(3 \mathrm{H}, \mathrm{s}, \mathrm{OCH}_{3}\right), 4.00(1 \mathrm{H}$, $\left.\mathrm{d},{ }^{2} J_{\mathrm{HH}}=15.7 \mathrm{~Hz}, \mathrm{NCH}_{2}\right), 4.85\left(1 \mathrm{H}, \mathrm{d},{ }^{2} J_{\mathrm{HH}}=15.7 \mathrm{~Hz}, \mathrm{NCH}_{2}\right), 4.90(1 \mathrm{H}$, $\left.\mathrm{dd},{ }^{3} J_{\mathrm{HF}}=14.4 \mathrm{~Hz},{ }^{3} J_{\mathrm{HH}}=6.5 \mathrm{~Hz}, \mathrm{CHCF}\right), 5.22\left(1 \mathrm{H}, \mathrm{ddm},{ }^{3} J_{\mathrm{HH}}=8.2 \mathrm{~Hz}, 7.4 \mathrm{~Hz}, \mathrm{CHCHCF}\right), 6.23(1 \mathrm{H}$, $\left.\mathrm{ddm},{ }^{3} J_{\mathrm{HH}}=9.2 \mathrm{~Hz}, 5.9 \mathrm{~Hz}, \mathrm{CH}=\mathrm{CHCHCF}\right), 6.30\left(1 \mathrm{H}, \mathrm{dd},{ }^{3} J_{\mathrm{HF}}=5.9 \mathrm{~Hz},{ }^{4} J_{\mathrm{HH}}=0.6 \mathrm{~Hz}, \mathrm{NC}=\mathrm{CH}\right), 6.81$ $\left(2 \mathrm{H}, \mathrm{dm},{ }^{3} J_{\mathrm{HH}}=8.8 \mathrm{~Hz}, \mathrm{PMB}\right), 7.09\left(2 \mathrm{H}, \mathrm{dm},{ }^{3} J_{\mathrm{HH}}=8.8 \mathrm{~Hz}, \mathrm{PMB}\right) .{ }^{13} \mathrm{C} \mathrm{NMR}(100 \mathrm{MHz}) \delta=52.1$, 55.2, 58.5, $59.7\left(\mathrm{~d},{ }^{2} J_{\mathrm{CF}}=21.6 \mathrm{~Hz}\right), 91.2(\mathrm{~m}), 92.2\left(\mathrm{~d},{ }^{3} J_{\mathrm{CF}}=6.9 \mathrm{~Hz}\right), 112.9\left(\mathrm{~d},{ }^{3} J_{\mathrm{CF}}=4.3 \mathrm{~Hz}\right), 113.6$, 113.9, $120.7\left(\mathrm{qd},{ }^{1} J_{\mathrm{CF}}=287.1 \mathrm{~Hz},{ }^{2} J_{\mathrm{CF}}=26.7 \mathrm{~Hz}\right), 127.2,129.1,135.8,159.1,164.3 .{ }^{19} \mathrm{~F}$ NMR $(376$ $\mathrm{MHz}) \delta=-186.19\left(1 \mathrm{~F}, \mathrm{~m},{ }^{3} J=7.8 \mathrm{~Hz}, \mathrm{CF}\left(\mathrm{CF}_{3}\right)_{2}\right),-73.55\left(3 \mathrm{~F}, \mathrm{~m},{ }^{3} J_{\mathrm{FF}}=8.5 \mathrm{~Hz}, \mathrm{CF}(\mathrm{CF})_{2}\right),-73.16(3 \mathrm{~F}$, $\left.\mathrm{m},{ }^{3} J_{\mathrm{FF}}=8.5 \mathrm{~Hz}, \mathrm{CF}\left(\mathrm{CF}_{3}\right)_{2}\right)$. MS (EI $\left.70 \mathrm{eV}\right) \mathrm{m} / z(\%): 427\left(\mathrm{M}^{+},<1\right), 258(11), 166(10), 121(100)$. HRMS (EI) : calcd for $\mathrm{C}_{18} \mathrm{H}_{16} \mathrm{NO}_{3} \mathrm{~F}_{7}\left(\mathrm{M}^{+}\right)$427.1018, found 427.1030. Anal. Calcd for $\mathrm{C}_{18} \mathrm{H}_{16} \mathrm{NO}_{3} \mathrm{~F}_{7}$ : C, 50.59; H, 3.77; N, 3.28; F, 31.12. Found : C, 51.14; H, 3.98; N, 3.09; F, 29.03.

\section{2-Heptafluoroisopropyl-1-(p-methoxybenzyl)-3,6-bis(methoxycarbonyl)-1,2-dihydropyridine}

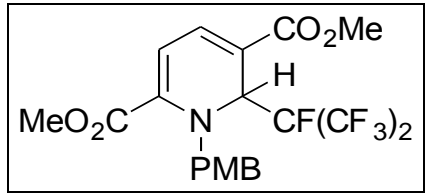

(2o'). Yellow crystalline solid, mp $44-45^{\circ} \mathrm{C}$ from hexanes/Et ${ }_{2} \mathrm{O}$. IR $\left(\mathrm{KBr}, v_{\max } / \mathrm{cm}^{-1}\right): 3025,2968,2846,1727,1707,1609,1515,1439,1281$, $1250,1224,1127,983,770 .{ }^{1} \mathrm{H}$ NMR $(400 \mathrm{MHz}) \delta=3.70\left(3 \mathrm{H}, \mathrm{s}, \mathrm{OCH}_{3}\right)$, $3.75\left(3 \mathrm{H}, \mathrm{s}, \mathrm{OCH}_{3}\right), 3.79\left(3 \mathrm{H}, \mathrm{s}, \mathrm{OCH}_{3}\right), 4.07\left(1 \mathrm{H}, \mathrm{d},{ }^{2} J_{\mathrm{HH}}=15.3 \mathrm{~Hz}\right.$, $\left.\mathrm{NCH}_{2}\right), 4.99\left(1 \mathrm{H}, \mathrm{d},{ }^{2} J_{\mathrm{HH}}=15.3 \mathrm{~Hz}, \mathrm{NCH}_{2}\right), 5.70\left(1 \mathrm{H}, \mathrm{d},{ }^{3} J_{\mathrm{HF}}=16.1 \mathrm{~Hz}, \mathrm{CHCF}\right), 6.45\left(1 \mathrm{H}, \mathrm{d},{ }^{3} J_{\mathrm{HH}}=6.1\right.$ $\mathrm{Hz}, \mathrm{NC}=\mathrm{CH}), 6.76\left(2 \mathrm{H}, \mathrm{dm},{ }^{3} J_{\mathrm{HH}}=8.7 \mathrm{~Hz}, \mathrm{PMB}\right), 6.96\left(2 \mathrm{H}, \mathrm{dm},{ }^{3} J_{\mathrm{HH}}=8.7 \mathrm{~Hz}, \mathrm{PMB}\right), 7.23(1 \mathrm{H}, \mathrm{d}$, $\left.{ }^{3} J_{\mathrm{HH}}=6.2 \mathrm{~Hz}, \mathrm{NC}=\mathrm{CHCH}\right) .{ }^{13} \mathrm{C} \mathrm{NMR}(100 \mathrm{MHz}) \delta=52.0,52.5,55.1,57.7\left(\mathrm{~d},{ }^{2} J_{\mathrm{CF}}=21.6 \mathrm{~Hz}\right), 58.9$, $91.7\left(\mathrm{dm},{ }^{1} J_{\mathrm{CF}}=212.8 \mathrm{~Hz},{ }^{2} J_{\mathrm{CF}}=29.3 \mathrm{~Hz}\right), 110.1,113.6,113.9,120.5\left(\mathrm{qd},{ }^{1} J_{\mathrm{CF}}=287.1 \mathrm{~Hz},{ }^{2} J_{\mathrm{CF}}=27.6\right.$ $\mathrm{Hz}), 120.6\left(\mathrm{qd},{ }^{1} J_{\mathrm{CF}}=288.8 \mathrm{~Hz},{ }^{2} J_{\mathrm{CF}}=27.6 \mathrm{~Hz}\right), 129.0,129.2,130.2,134.5,139.2,159.3,163.3,165.5$. ${ }^{19} \mathrm{~F}$ NMR $(376 \mathrm{MHz}) \delta=-189.90\left(1 \mathrm{~F}, \mathrm{~m},{ }^{3} \mathrm{~J}=7.9 \mathrm{~Hz}, \mathrm{CF}\left(\mathrm{CF}_{3}\right)_{2}\right),-73.79\left(3 \mathrm{~F}, \mathrm{~m}, \mathrm{CF}(\mathrm{CF})_{2}\right),-72.28(3 \mathrm{~F}$, m, $\left.\mathrm{CF}\left(\mathrm{CF}_{3}\right)_{2}\right)$. MS (EI $\left.70 \mathrm{eV}\right) \mathrm{m} / z(\%): 485\left(\mathrm{M}^{+},<1\right), 316(7), 121(100)$. HRMS (EI) : calcd for $\mathrm{C}_{20} \mathrm{H}_{18} \mathrm{NO}_{5} \mathrm{~F}_{7}\left(\mathrm{M}^{+}\right)$485.1073, found 485.1078. Anal. Calcd for $\mathrm{C}_{20} \mathrm{H}_{18} \mathrm{NO}_{5} \mathrm{~F}_{7}$ : C, 49.49; H, 3.74; N, 2.89; F, 27.40. Found : C, 49.71; H, 3.77; N, 3.02; F, 27.53. 


\section{4-Heptafluoroisopropyl-1-(p-methoxybenzyl)-2,5-bis(methoxycarbonyl)-1,4-dihydropyridine}

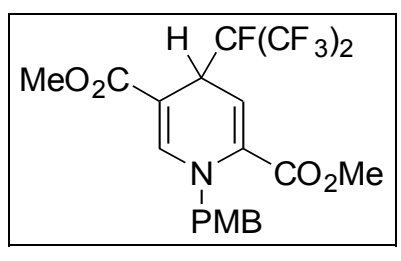

(3o'). Yellow crystalline solid, mp $64-65^{\circ} \mathrm{C}$ from hexanes/ $\mathrm{Et}_{2} \mathrm{O}$. IR $(\mathrm{KBr}$, $\left.\nu_{\max } / \mathrm{cm}^{-1}\right): 3020,2997,2951,2846,1731,1708,1658,1606,1515,1416$, $1313,1284,1255,1220,1120,1106,1053,981,954.912,842,754 .{ }^{1} \mathrm{H}$ NMR $(400 \mathrm{MHz}) \delta=3.72\left(3 \mathrm{H}, \mathrm{s}, \mathrm{OCH}_{3}\right), 3.73\left(3 \mathrm{H}, \mathrm{s}, \mathrm{OCH}_{3}\right), 3.80(3 \mathrm{H}, \mathrm{s}$, $\left.\mathrm{OCH}_{3}\right), 4.73\left(1 \mathrm{H}, \mathrm{t},{ }^{3} J=7.4 \mathrm{~Hz}, \mathrm{CHCF}\right), 4.79\left(2 \mathrm{H}, \mathrm{AB},{ }^{2} J_{\mathrm{HH}}=15.2 \mathrm{~Hz}, \mathrm{NCH}_{2}\right), 5.65\left(1 \mathrm{H}, \mathrm{d},{ }^{3} J_{\mathrm{HH}}=6.5\right.$ $\mathrm{Hz}, \mathrm{CHCHCF}), 6.86\left(2 \mathrm{H}, \mathrm{dm},{ }^{3} J_{\mathrm{HH}}=8.8 \mathrm{~Hz}, \mathrm{PMB}\right), 7.13\left(2 \mathrm{H}, \mathrm{dm},{ }^{3} J_{\mathrm{HH}}=8.8 \mathrm{~Hz}, \mathrm{PMB}\right), 7.47(1 \mathrm{H}, \mathrm{d}$, $\left.{ }^{4} J_{\mathrm{HH}}=0.8 \mathrm{~Hz}, \mathrm{NCH}=\mathrm{CCO}_{2} \mathrm{Me}\right) .{ }^{13} \mathrm{C} \mathrm{NMR}(100 \mathrm{MHz}) \delta=35.6\left(\mathrm{~d},{ }^{2} J_{\mathrm{CF}}=21.6 \mathrm{~Hz}\right), 51.5,52.7,54.9$, 55.2, $91.2\left(\mathrm{dm},{ }^{1} J_{\mathrm{CF}}=209.5 \mathrm{~Hz},{ }^{2} J_{\mathrm{CF}}=29.3 \mathrm{~Hz}\right), 93.9\left(\mathrm{~d},{ }^{3} J_{\mathrm{CF}}=4.3 \mathrm{~Hz}\right), 106.9\left(\mathrm{~d},{ }^{3} J_{\mathrm{CF}}=5.2 \mathrm{~Hz}\right), 114.1$, $120.7\left(\mathrm{qd},{ }^{1} J_{\mathrm{CF}}=288.0 \mathrm{~Hz},{ }^{2} J_{\mathrm{CF}}=28.4 \mathrm{~Hz}\right), 121.0\left(\mathrm{qd},{ }^{1} J_{\mathrm{CF}}=287.9 \mathrm{~Hz},{ }^{2} J_{\mathrm{CF}}=28.4 \mathrm{~Hz}\right), 128.1,129.2$, 135.1, 144.8, 159.4, 162.9, 167.5. ${ }^{19} \mathrm{~F}$ NMR (376 MHz) $\delta=-184.81\left(1 \mathrm{~F}, \mathrm{~m},{ }^{3} J=7.9 \mathrm{~Hz}, \mathrm{CF}\left(\mathrm{CF}_{3}\right)_{2}\right)$, $-75.49\left(3 \mathrm{~F}, \mathrm{~m},{ }^{3} J_{\mathrm{FF}}=8.7 \mathrm{~Hz}, \mathrm{CF}(\mathrm{CF})_{2}\right),-73.29\left(3 \mathrm{~F}, \mathrm{~m},{ }^{3} J_{\mathrm{FF}}=7.9 \mathrm{~Hz}, \mathrm{CF}\left(\mathrm{CF}_{3}\right)_{2}\right)$. MS $(\mathrm{EI} 70 \mathrm{eV}) \mathrm{m} / z$ (\%) : 485(M $\left(\mathrm{M}^{+},<1\right), 316(10), 121(100)$. HRMS (EI) : calcd for $\mathrm{C}_{20} \mathrm{H}_{18} \mathrm{NO}_{5} \mathrm{~F}_{7}\left(\mathrm{M}^{+}\right)$485.1073, found 485.1079. Anal. Calcd for $\mathrm{C}_{20} \mathrm{H}_{18} \mathrm{NO}_{5} \mathrm{~F}_{7}: \mathrm{C}, 49.49 ; \mathrm{H}, 3.74 ; \mathrm{N}, 2.89 ; \mathrm{F}, 27.40$. Found : C, 49.49; H, 3.77 ;, $2.72 ; \mathrm{F}, 26.18$. 


\section{Compound 3a: ${ }^{1} \mathrm{H}$ NMR}

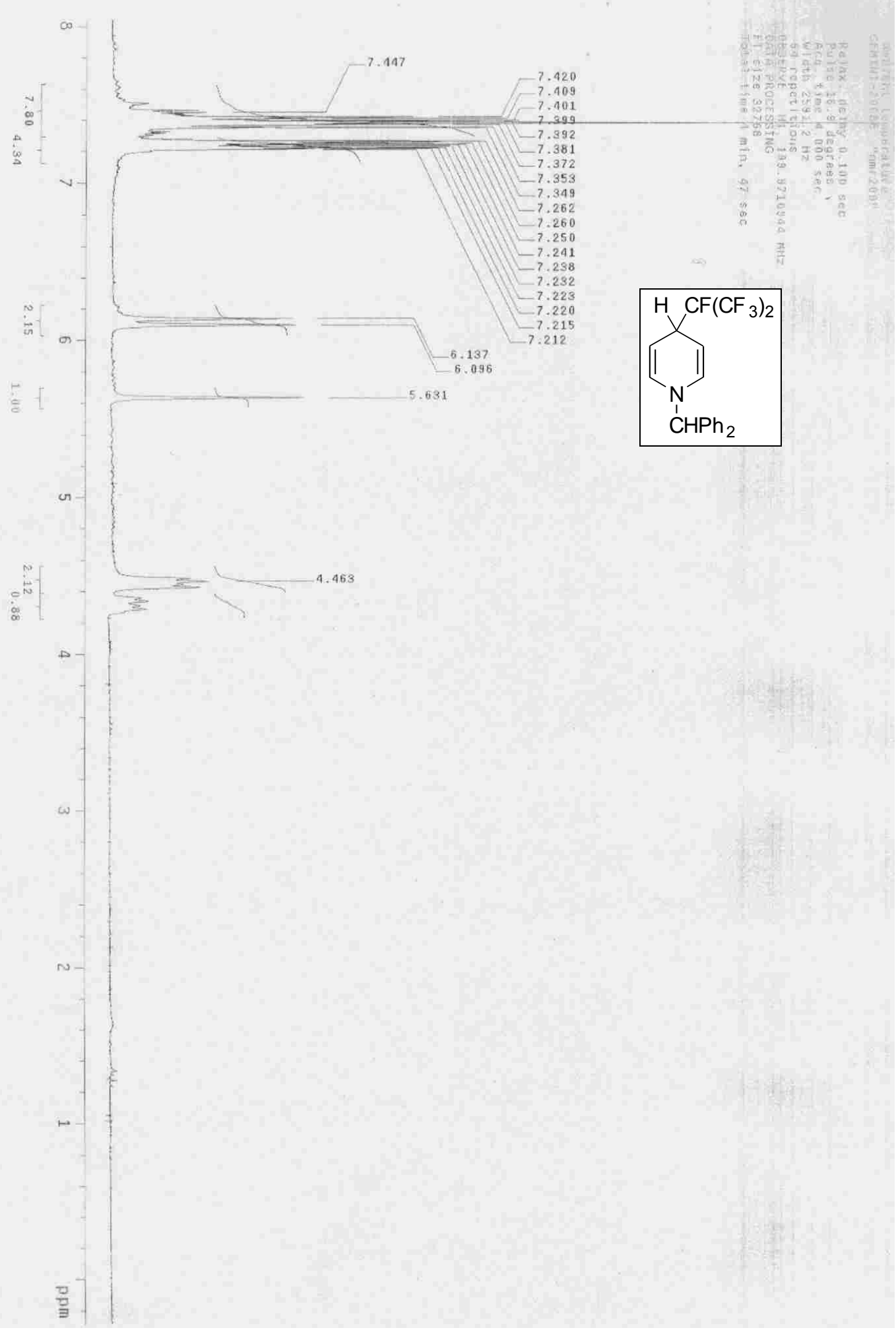




\section{Compound 3a: ${ }^{13} \mathrm{C}$ NMR}

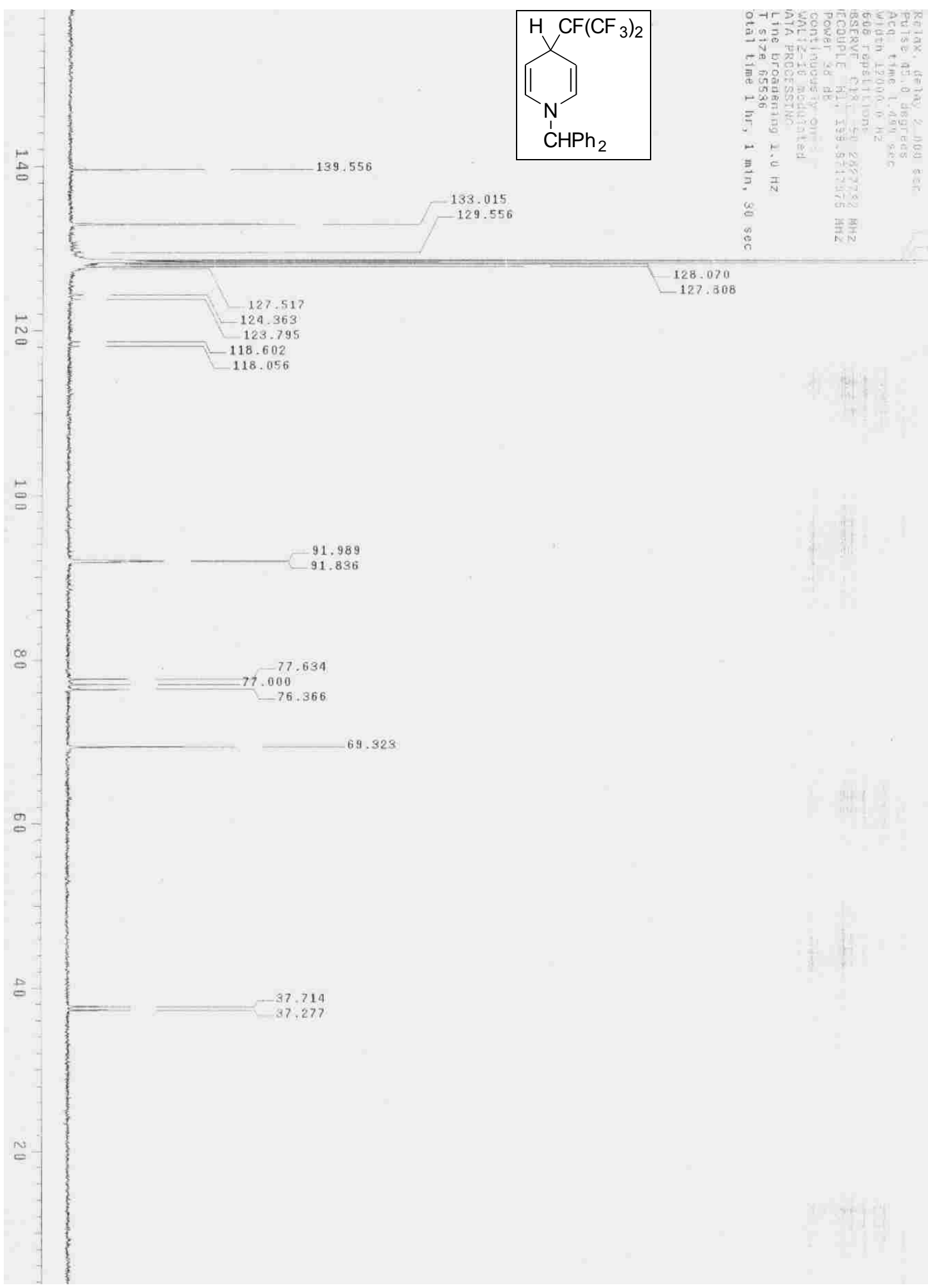




\section{Compound 4a: ${ }^{1} \mathrm{H}$ NMR}

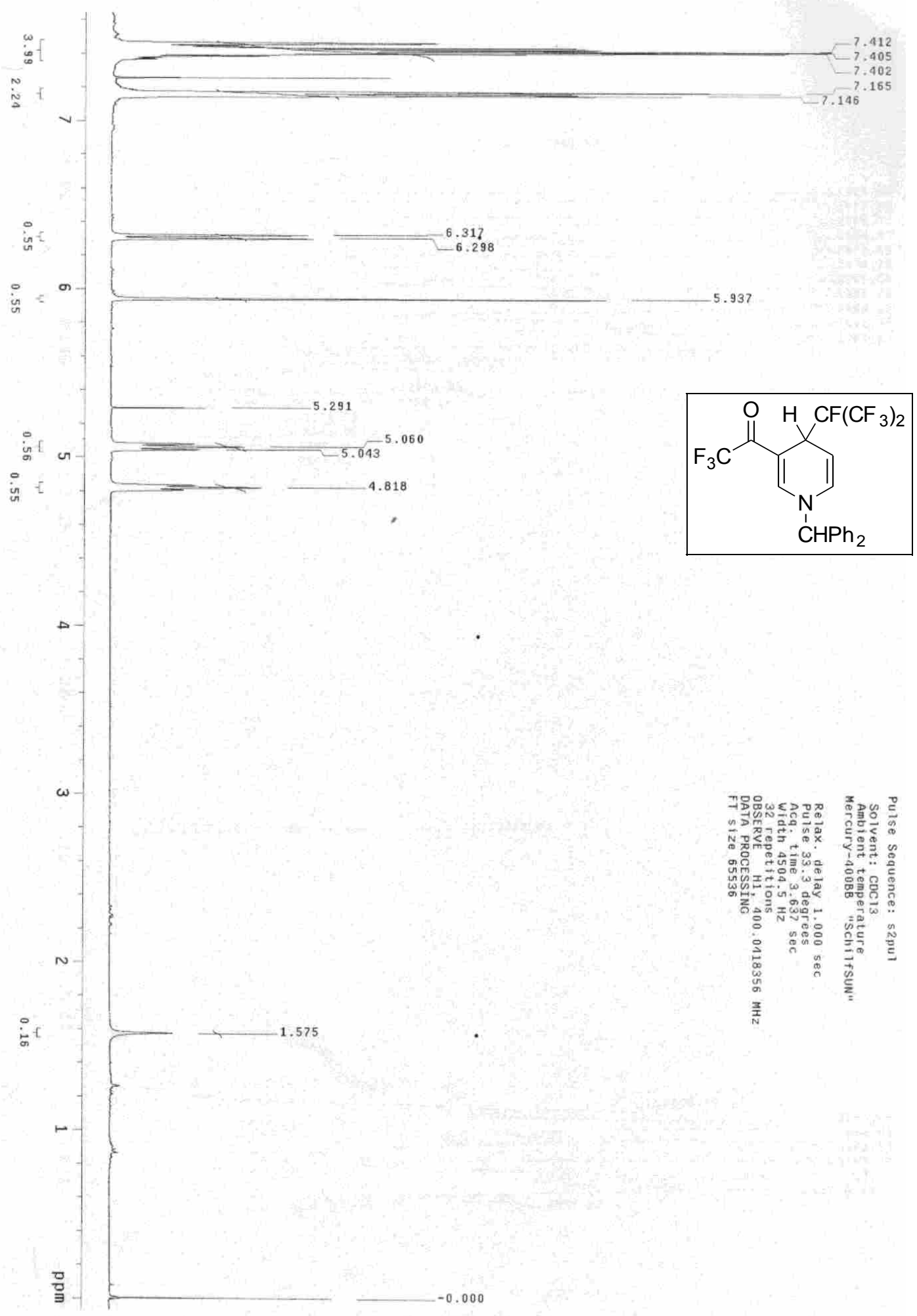


Compound 4a: ${ }^{13} \mathrm{C}$ NMR
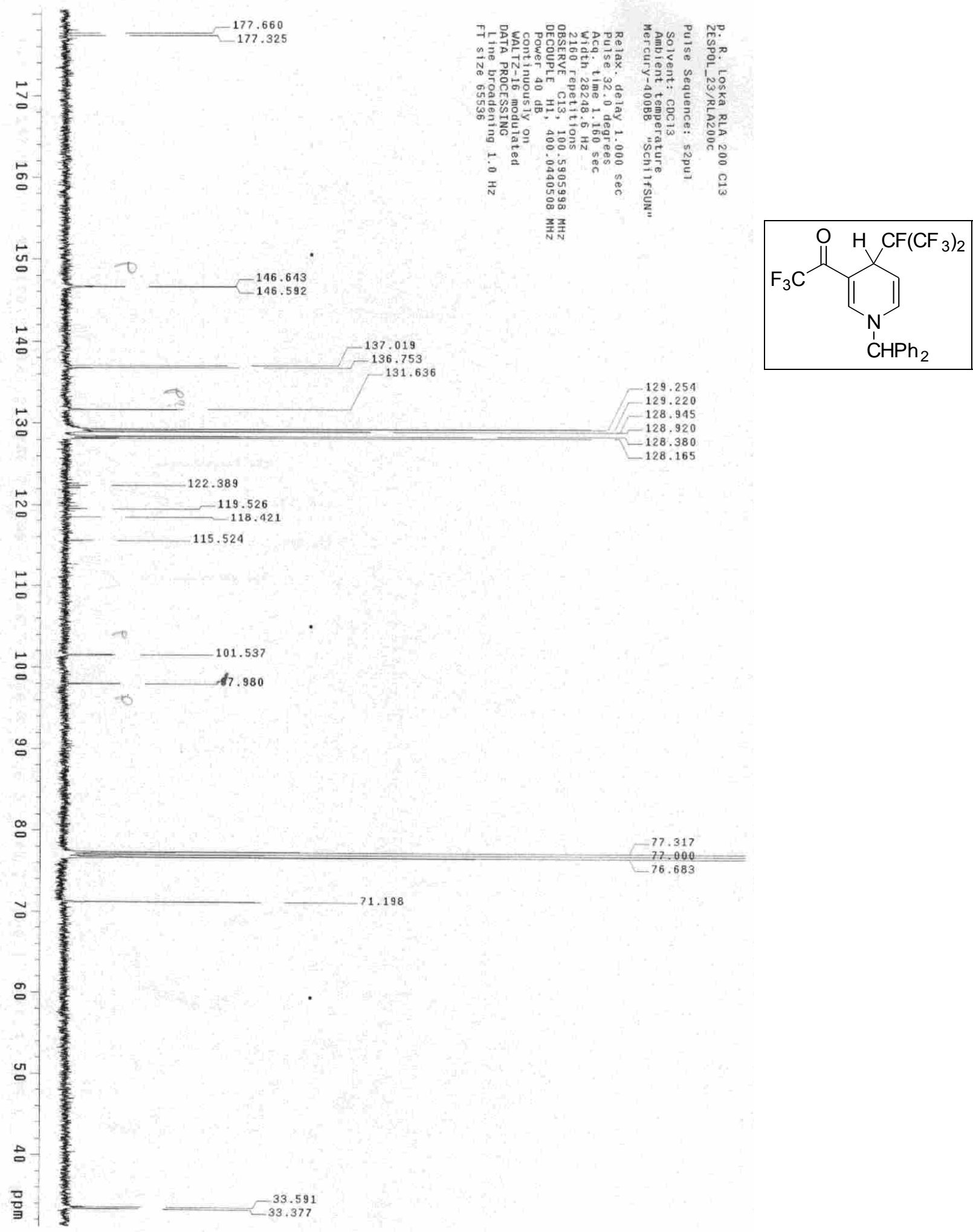


\section{Compound 4a: ${ }^{19}$ F NMR}

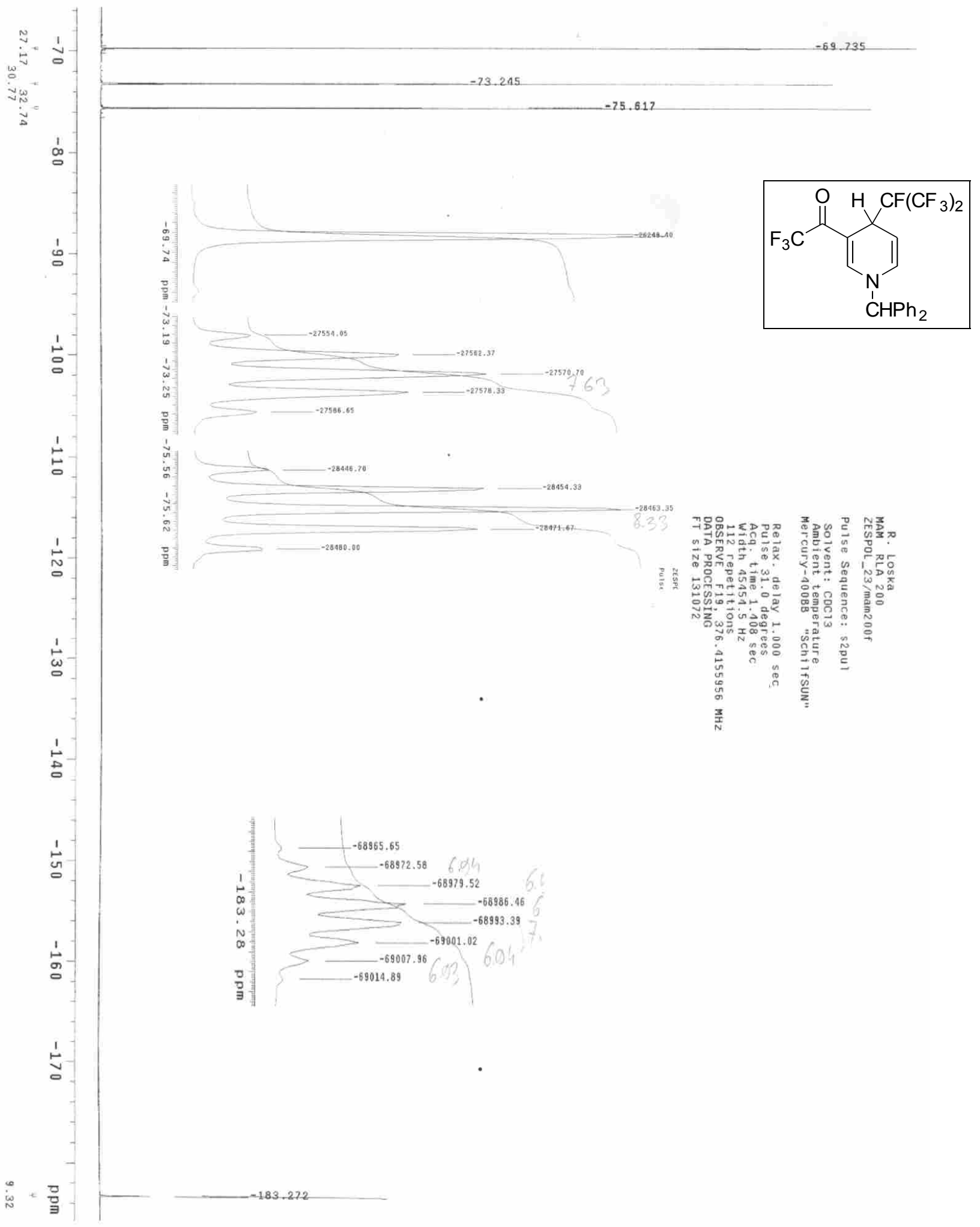




\section{Compound 4a: ${ }^{1} \mathrm{H}-{ }^{1} \mathrm{H}$ COSY}

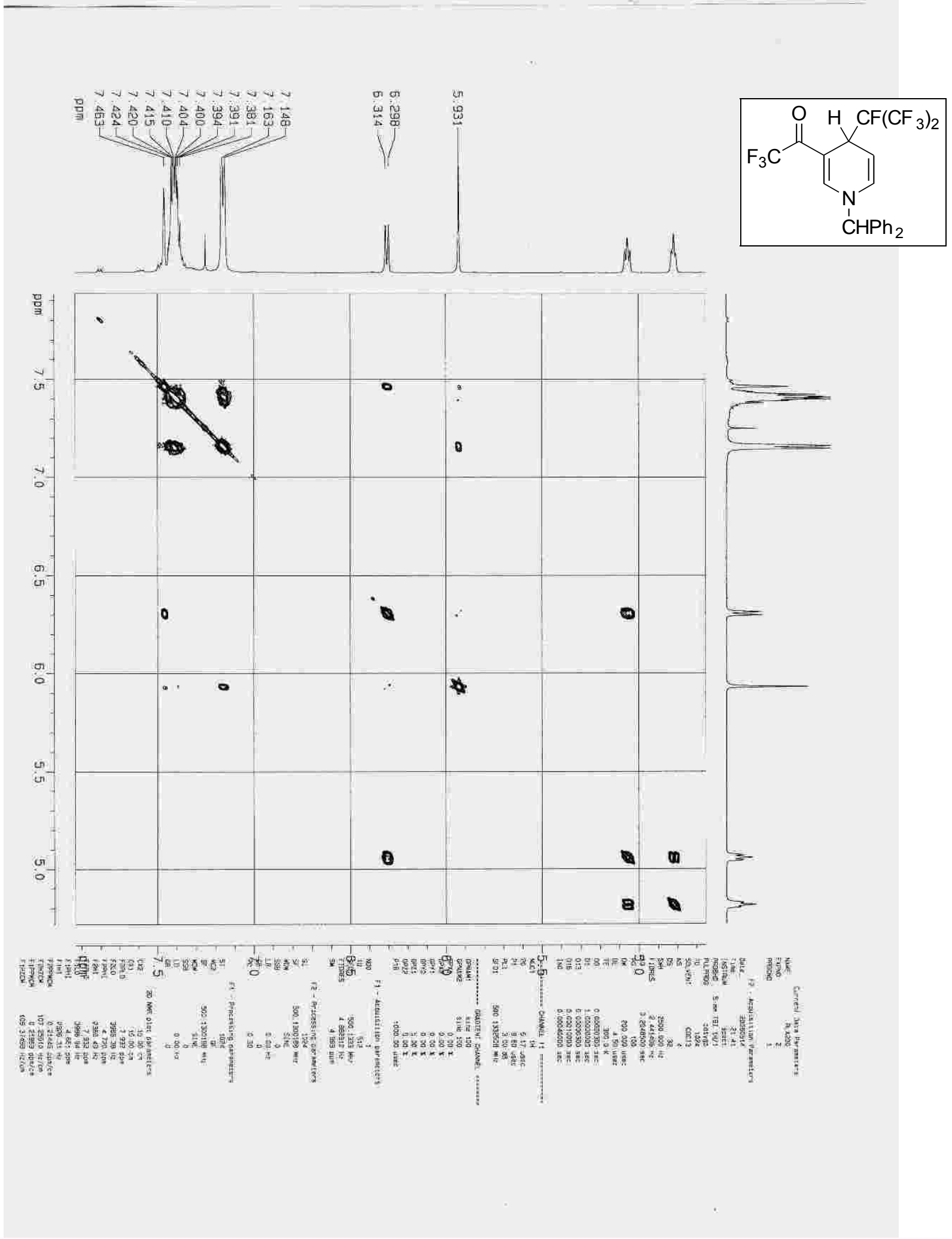




\section{Compound 4a: DEPT}

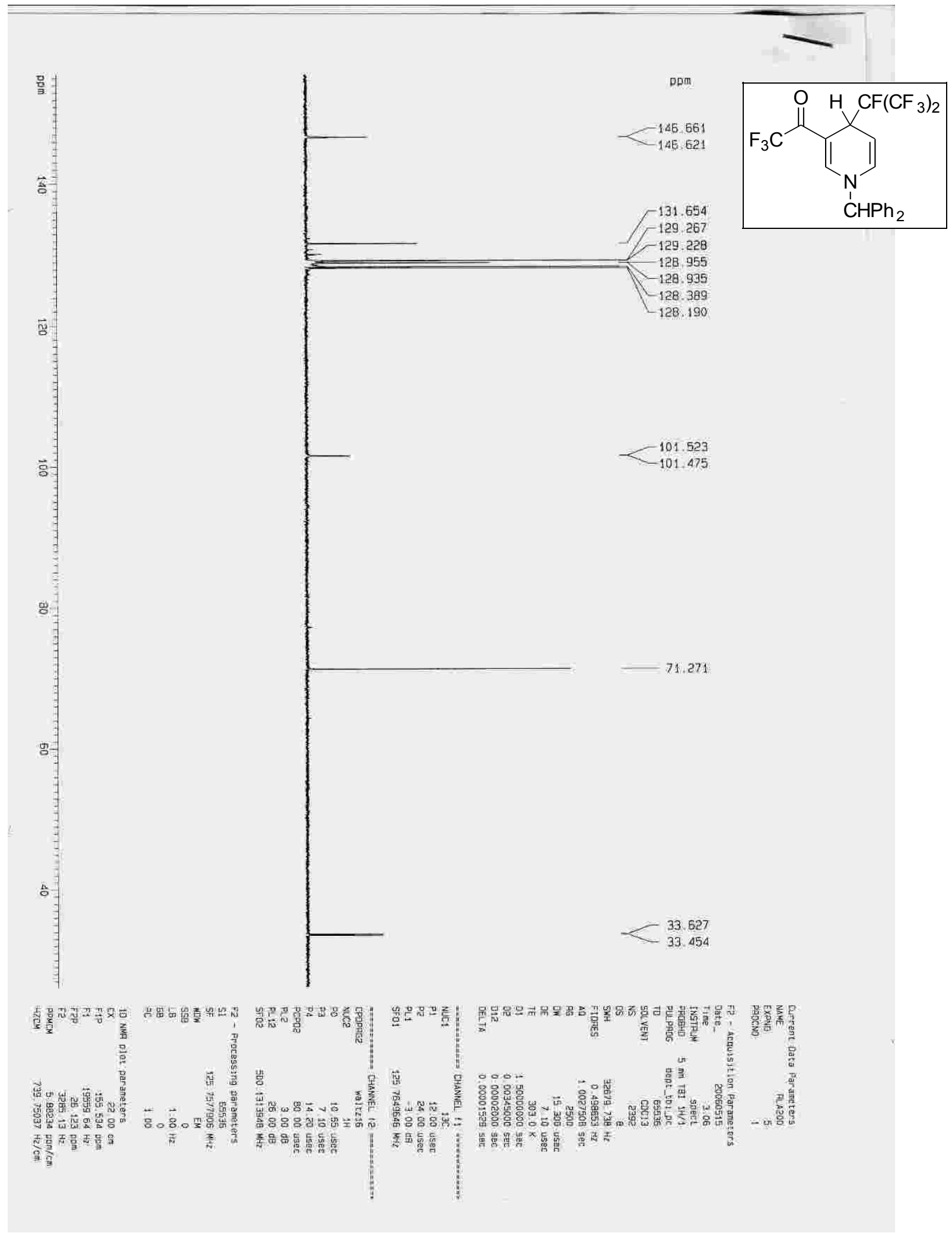




\section{Compound 4a: HSQC}

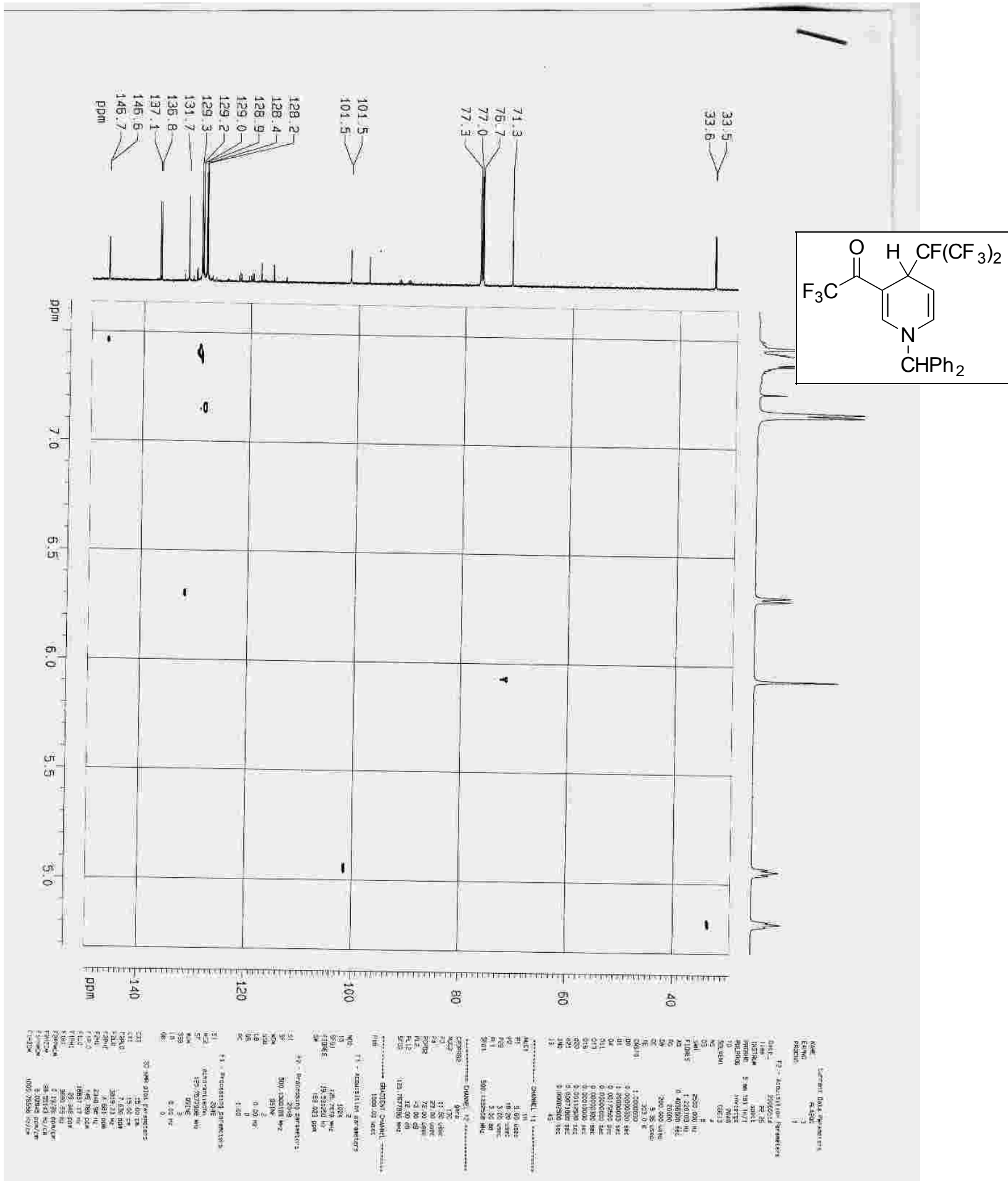




\section{Compound 4a: HMBC}

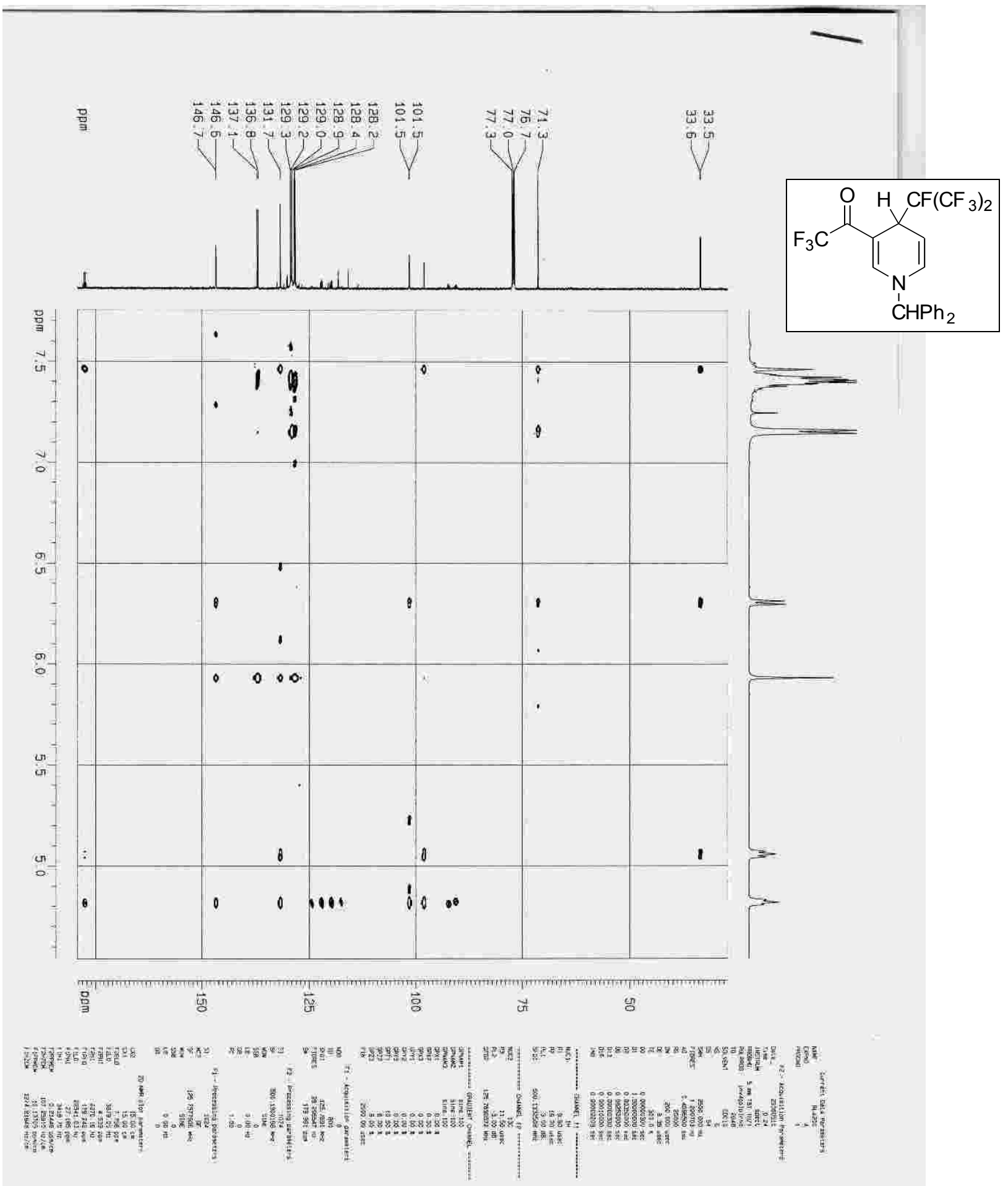




\section{Compound 3b: ${ }^{1} \mathrm{H}$ NMR}

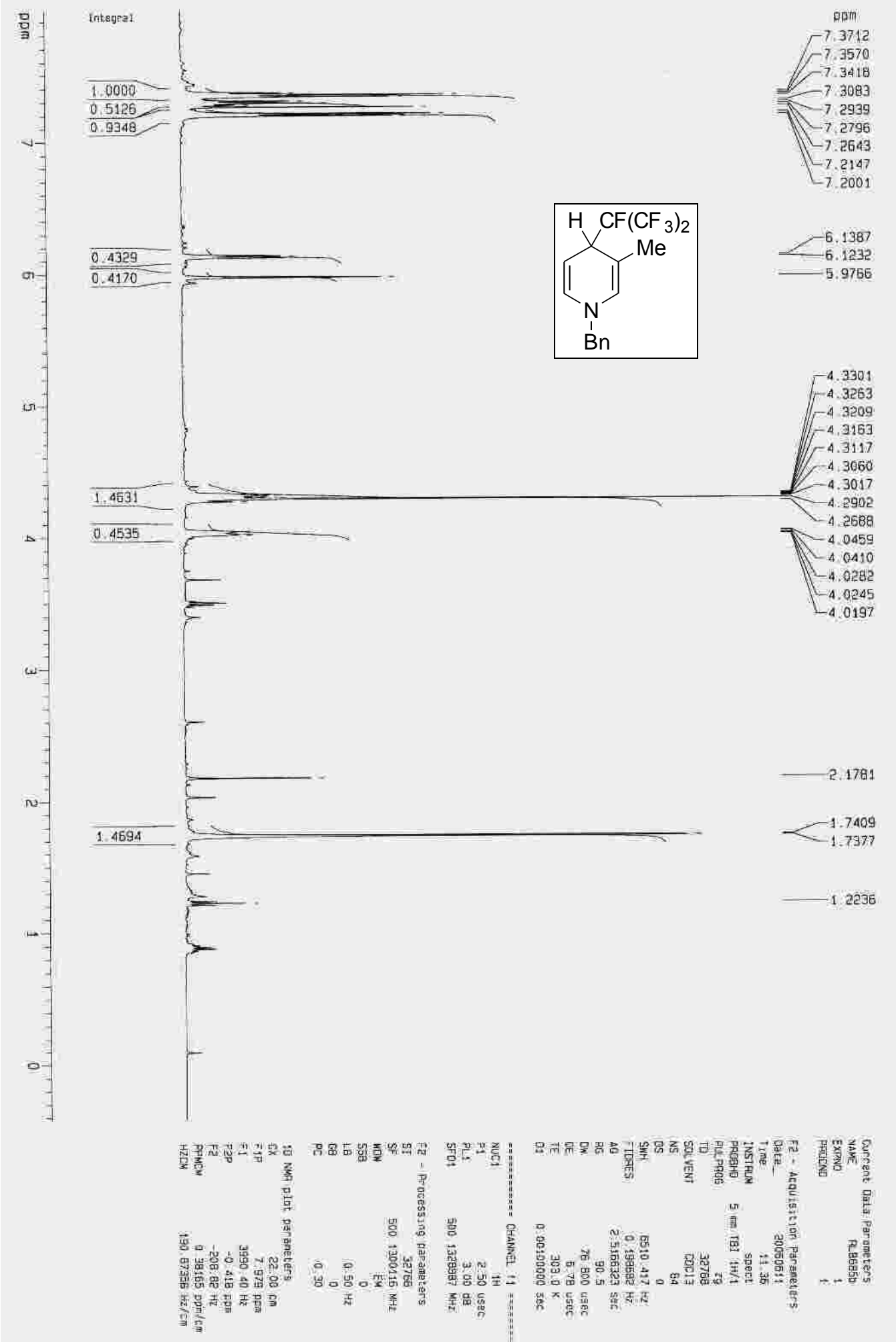




\section{Compound 3b: ${ }^{13} \mathrm{C}$ NMR}

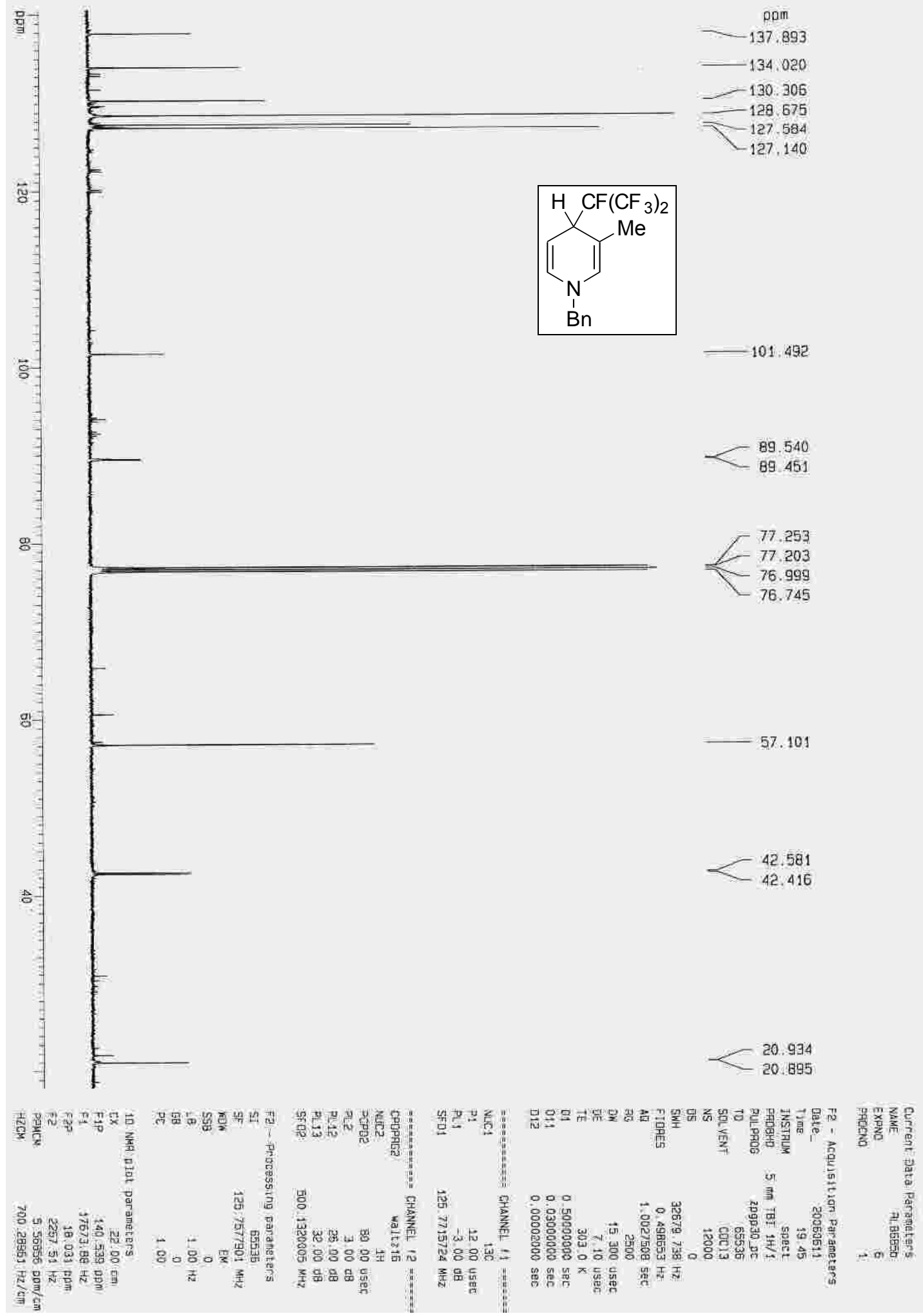


Compound 3b: ${ }^{1} \mathrm{H}-{ }^{1} \mathrm{H}$ COSY

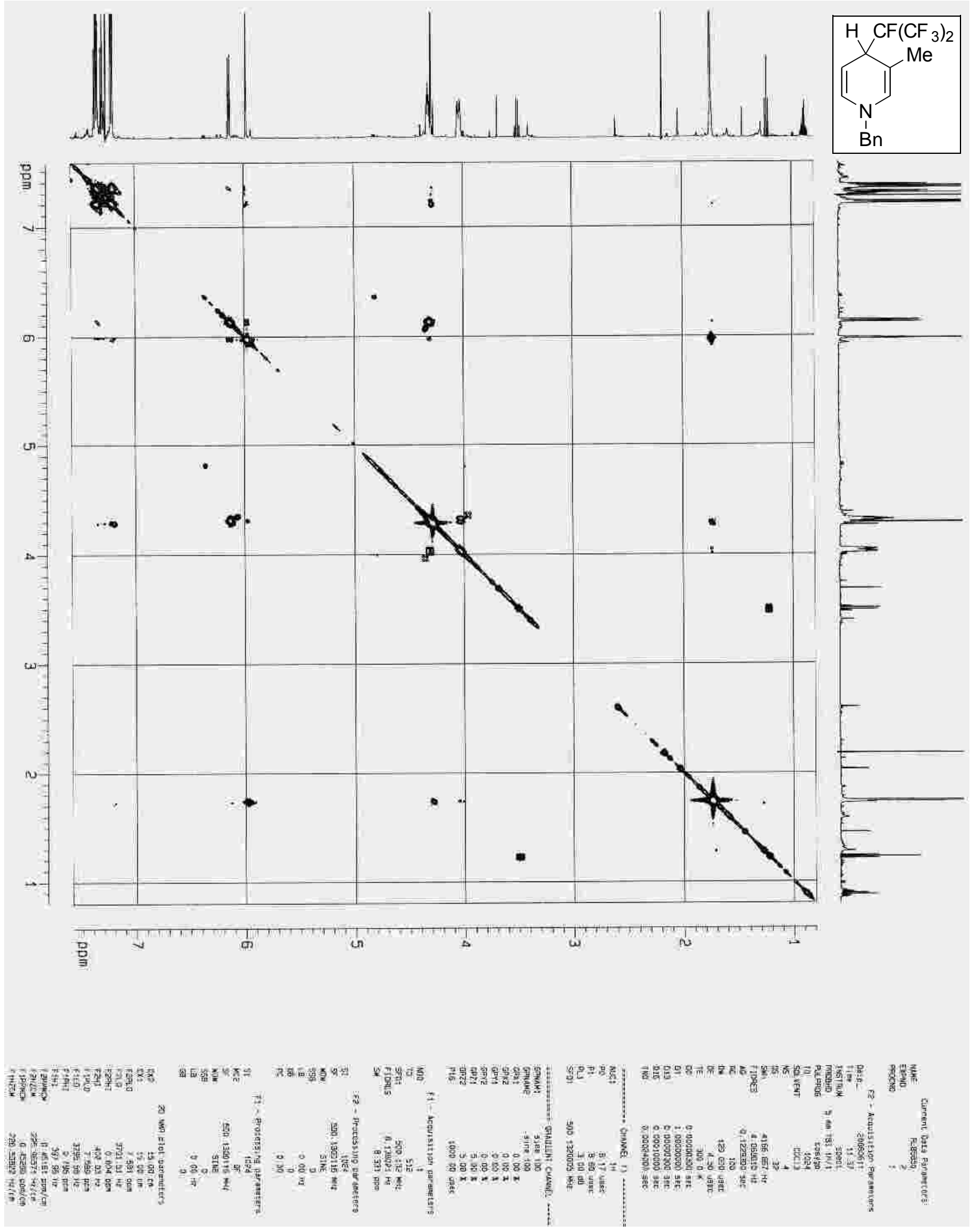




\section{Compound 3b: DEPT}

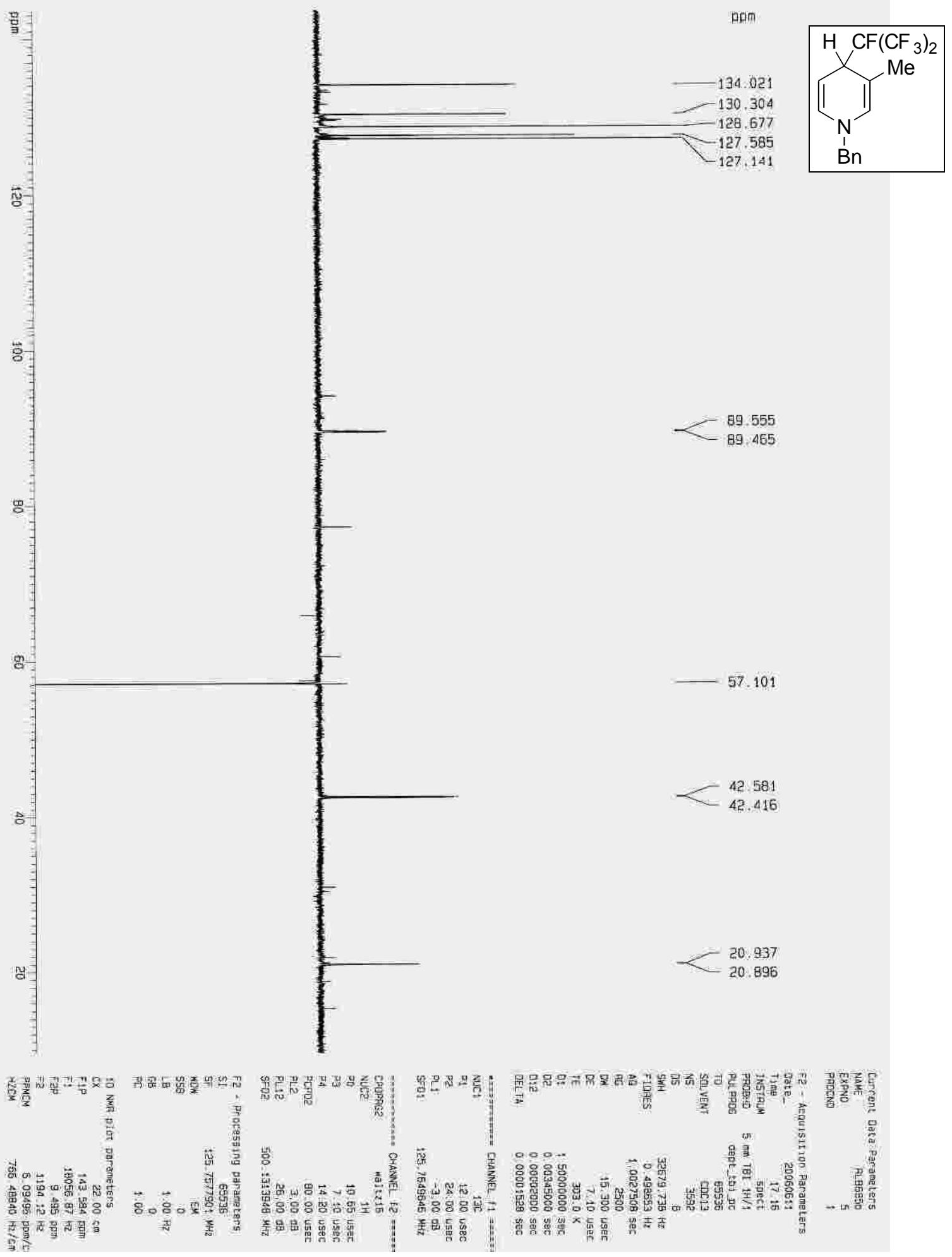




\section{Compound 3b: HSQC}

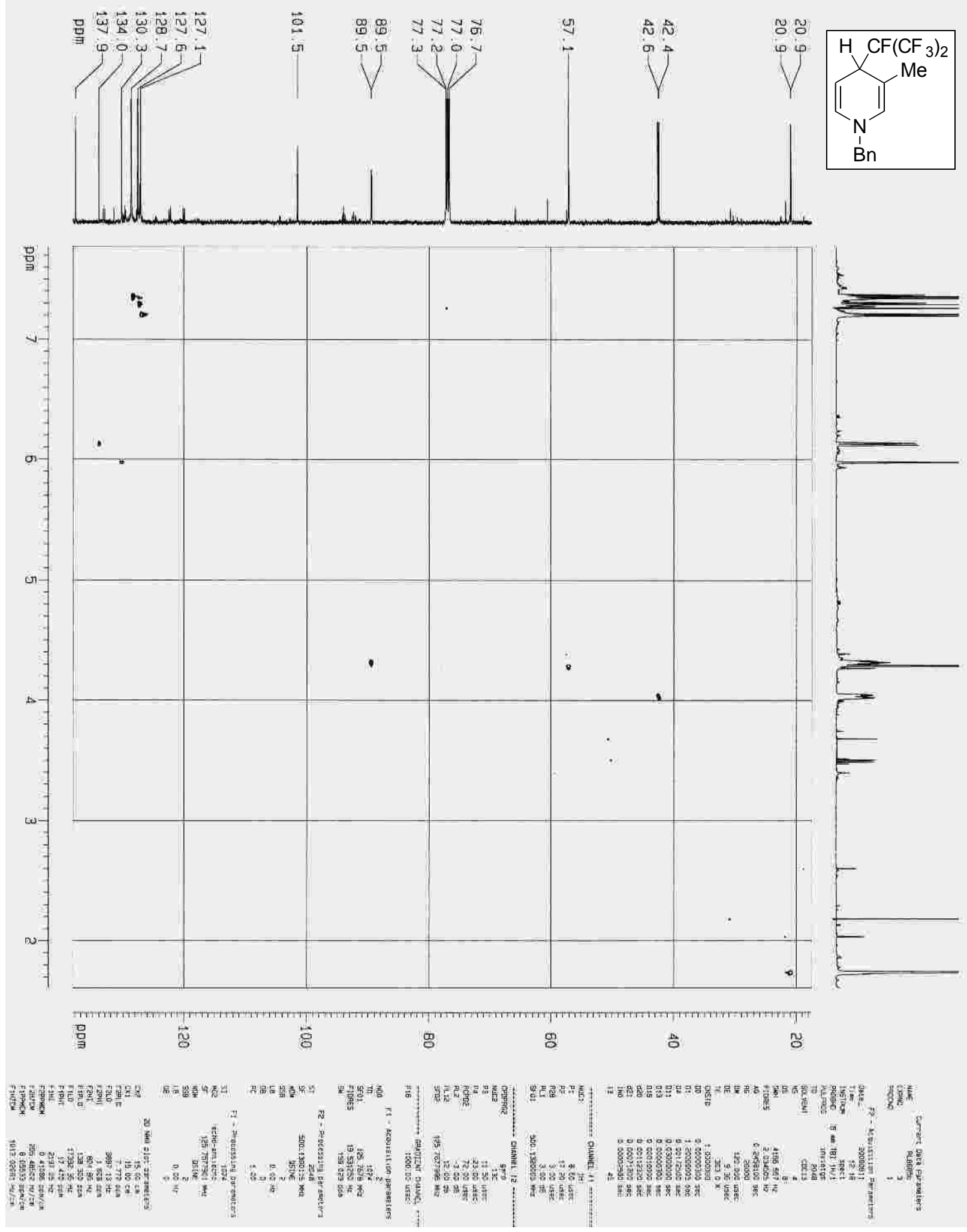


Compound 3b: HMBC

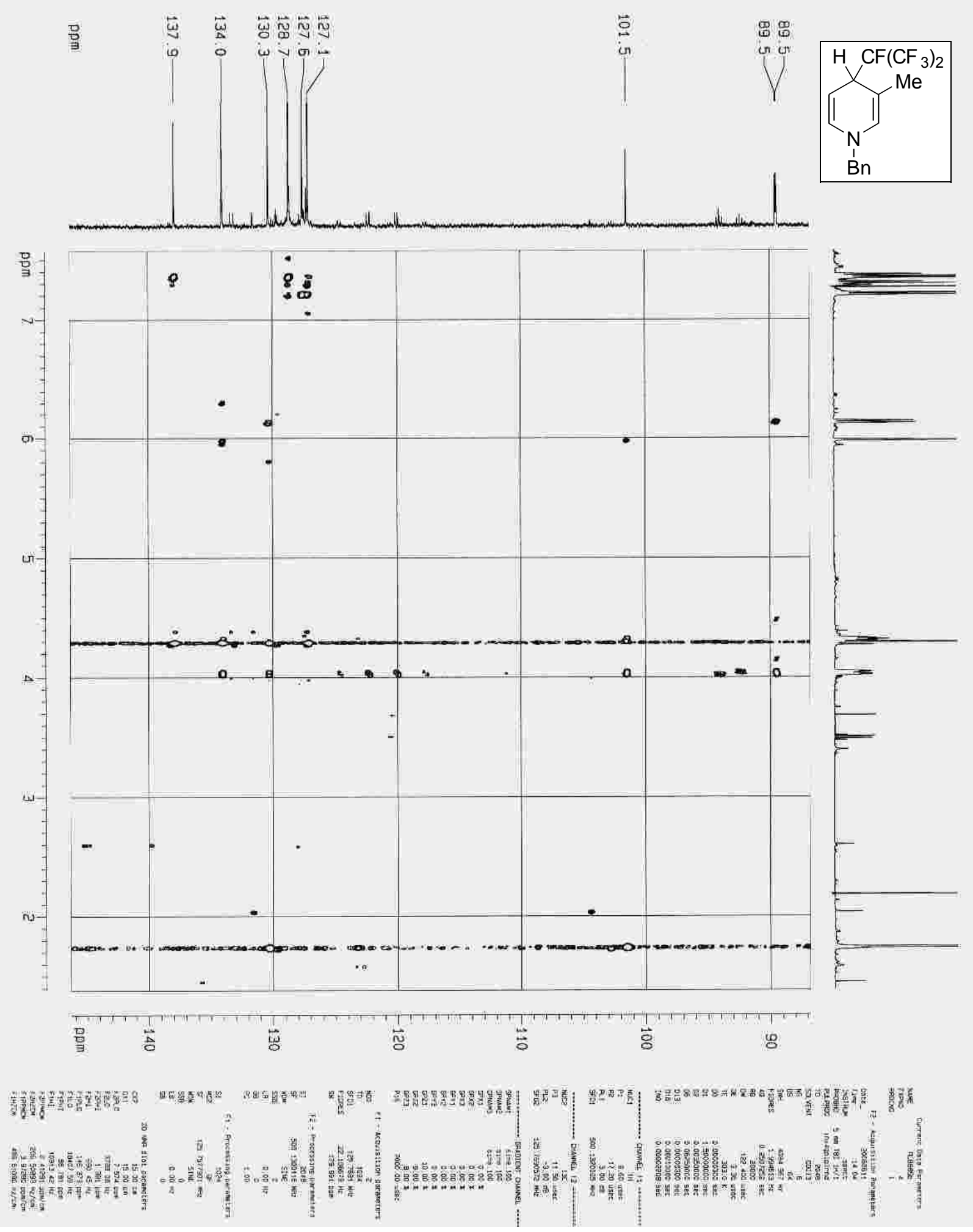




\section{Compound 4b: ${ }^{1} \mathrm{H}$ NMR}

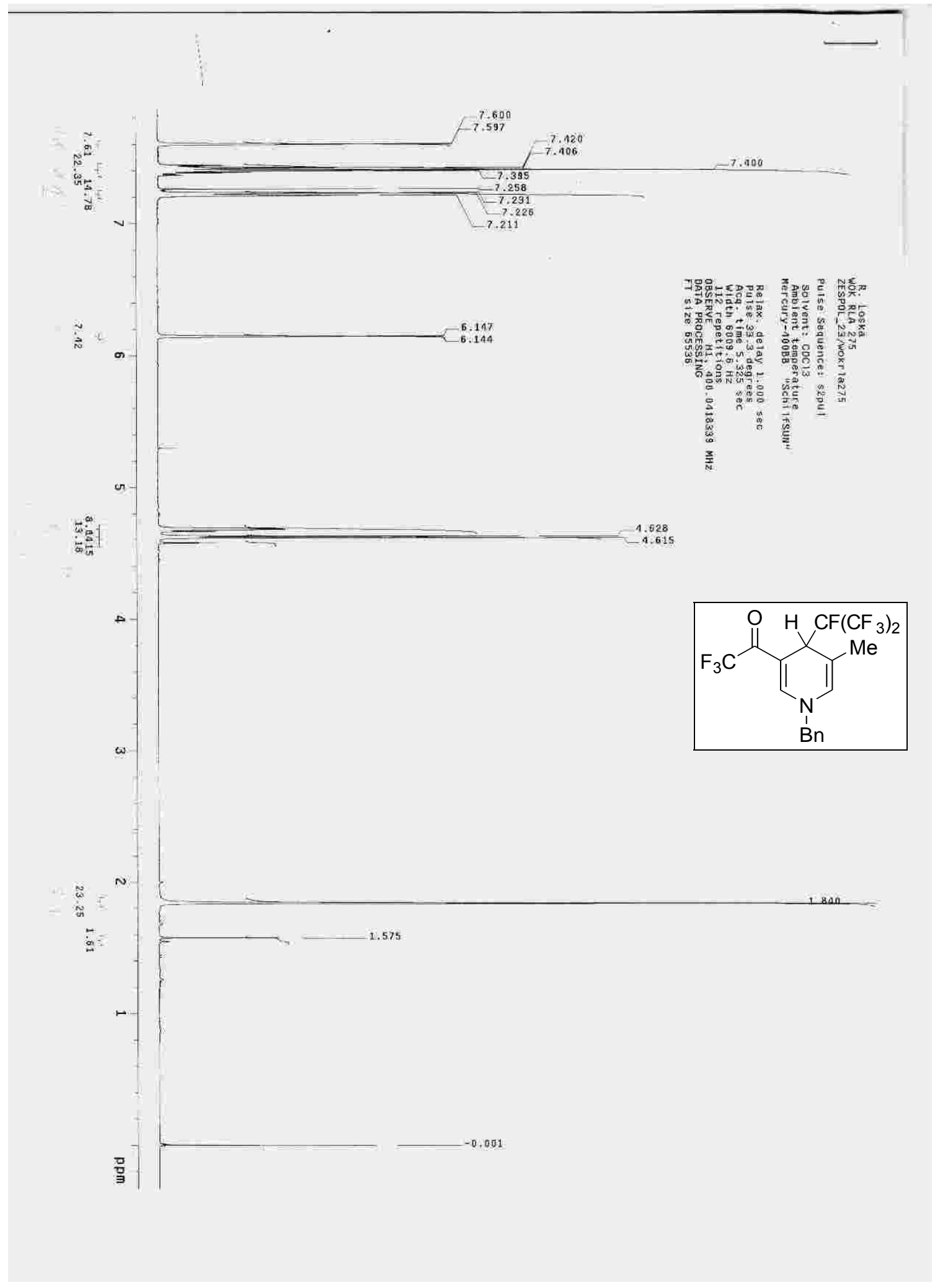


Compound 4b: ${ }^{13} \mathrm{C}$ NMR

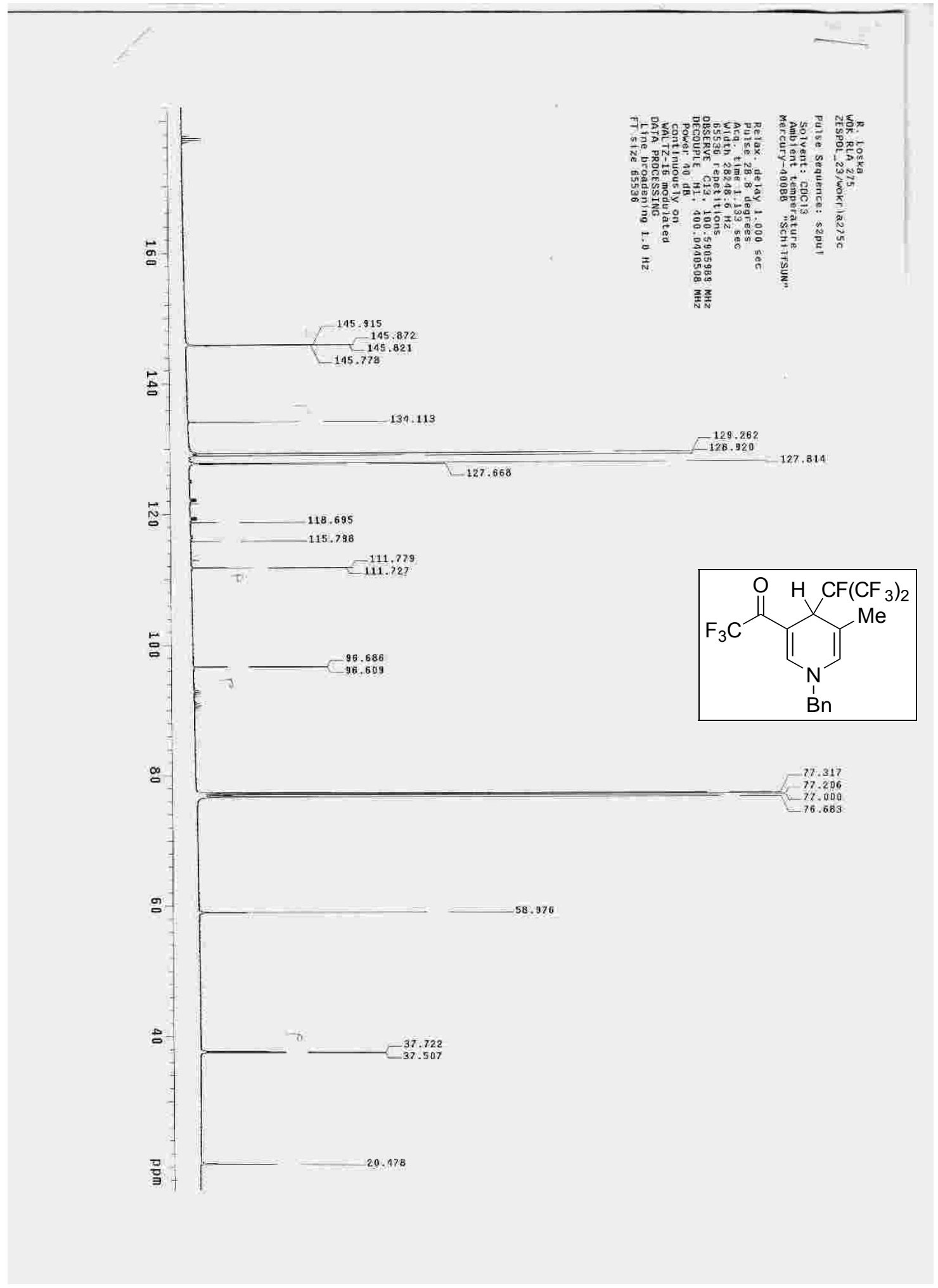




\section{Compound 4b: ${ }^{19} \mathrm{~F}$ NMR}

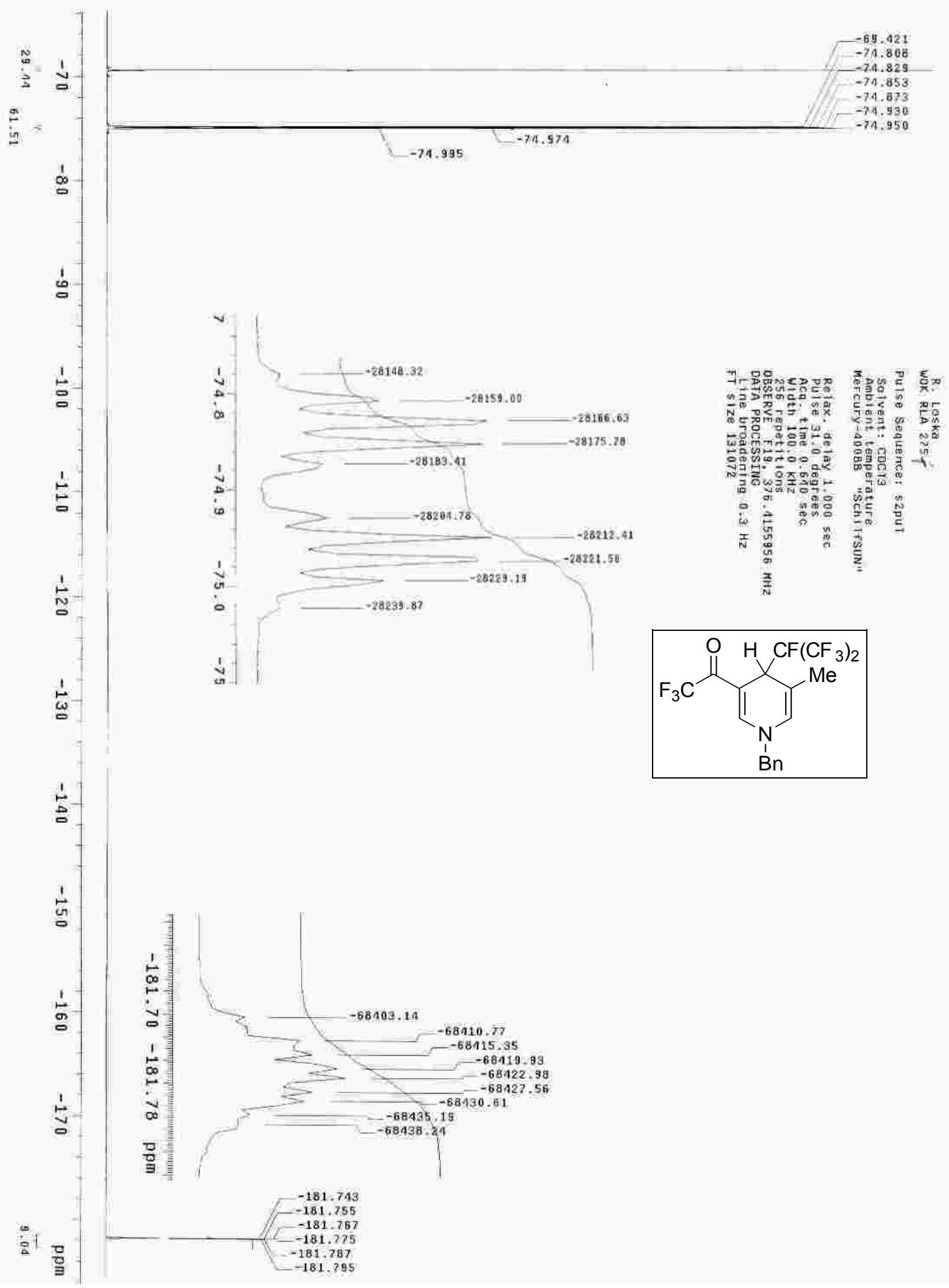




\section{Compound 2c: ${ }^{1} \mathrm{H}$ NMR}

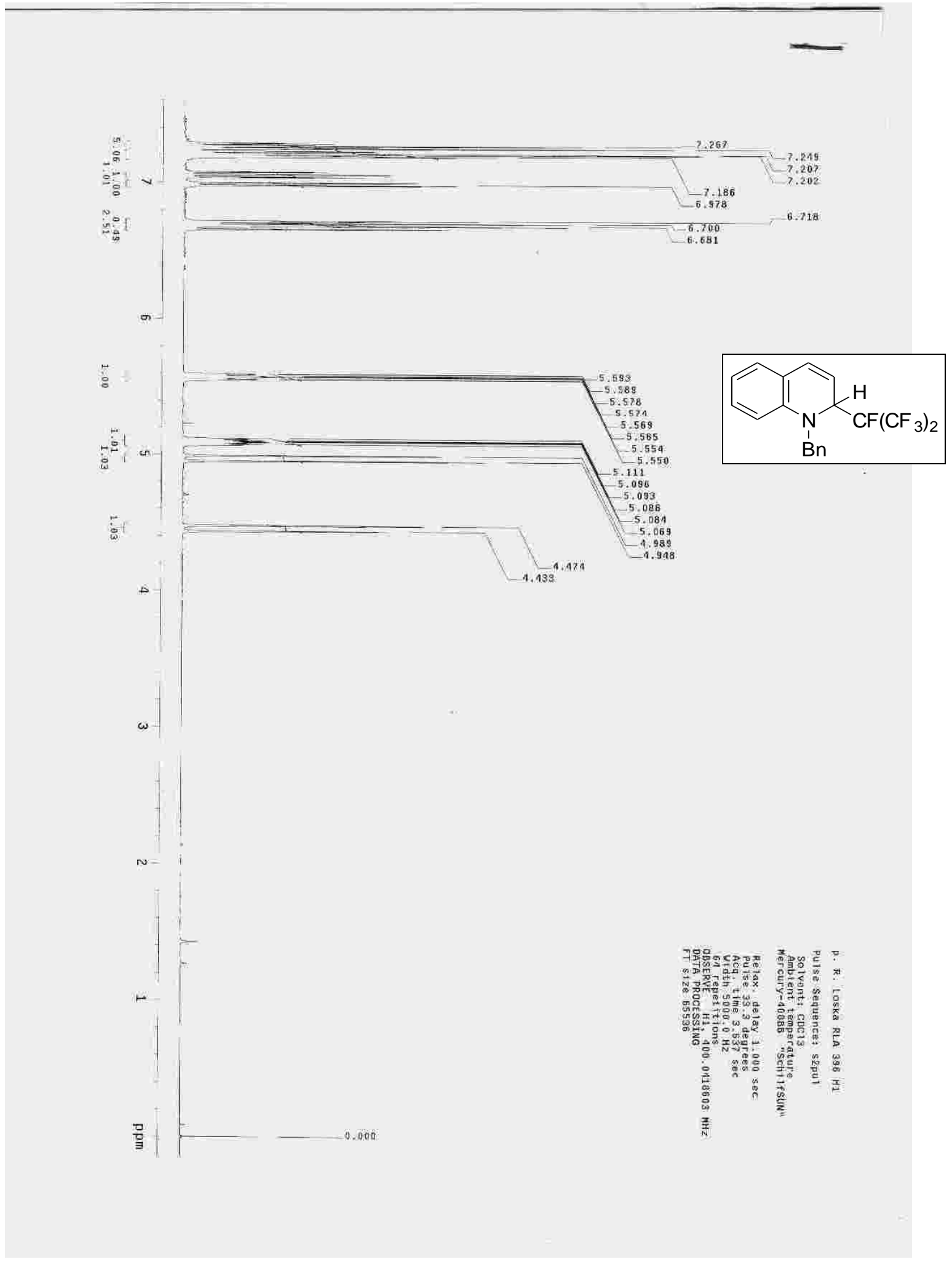




\section{Compound 2c: ${ }^{13} \mathrm{C}$ NMR}

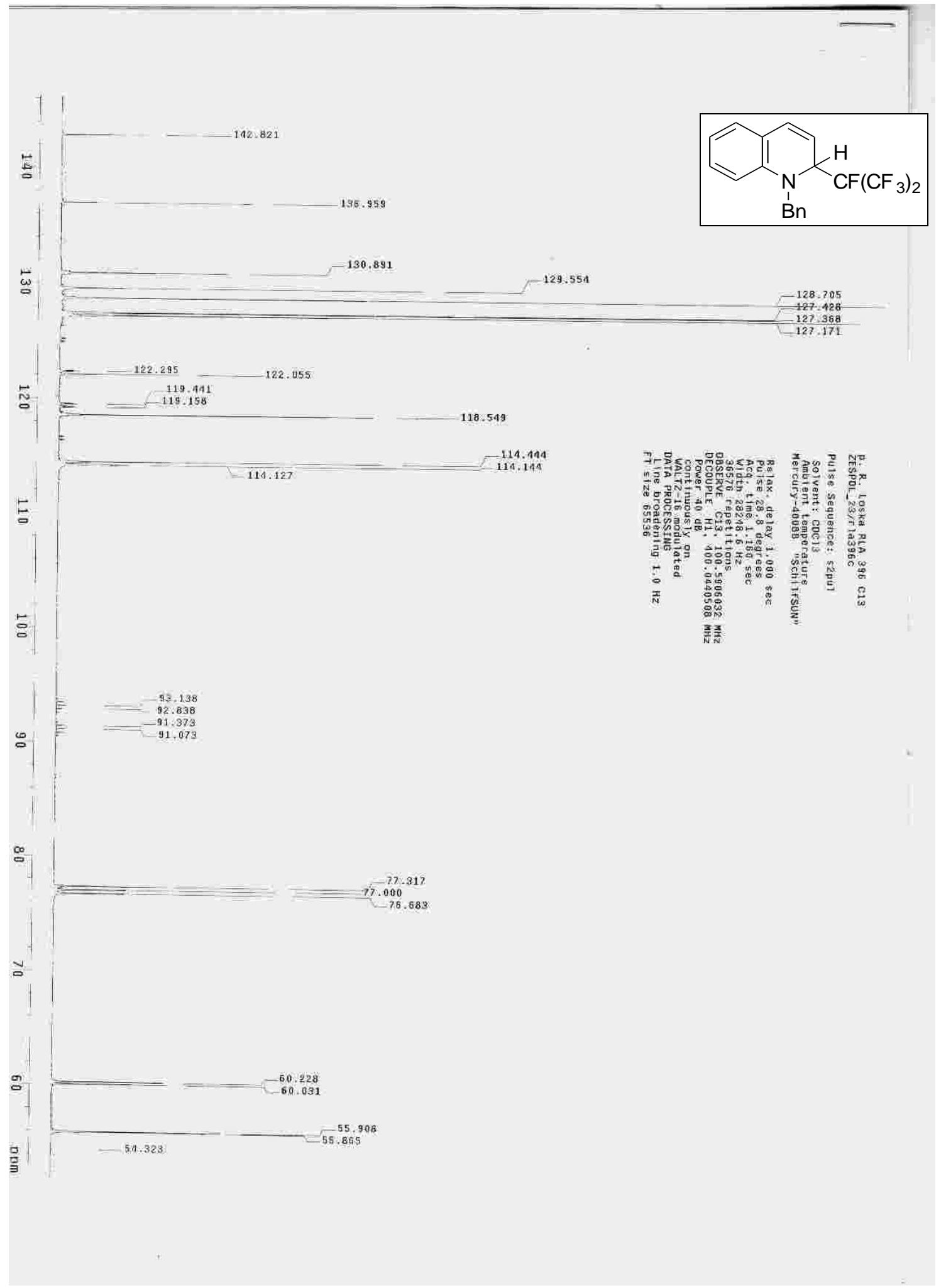


Compound 2c: ${ }^{19} \mathrm{~F}$ NMR

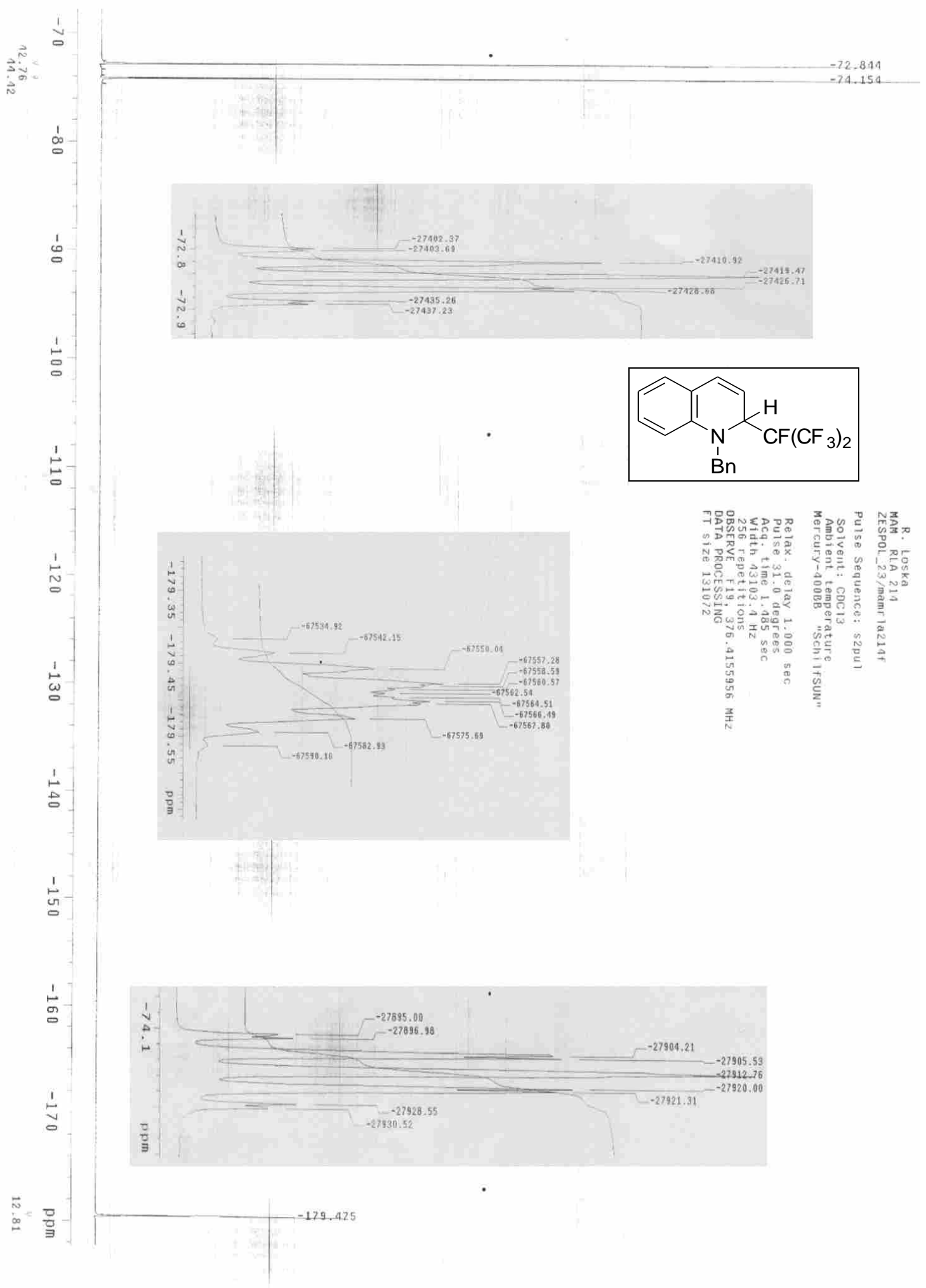




\section{Compound 2d: ${ }^{1} \mathrm{H}$ NMR}

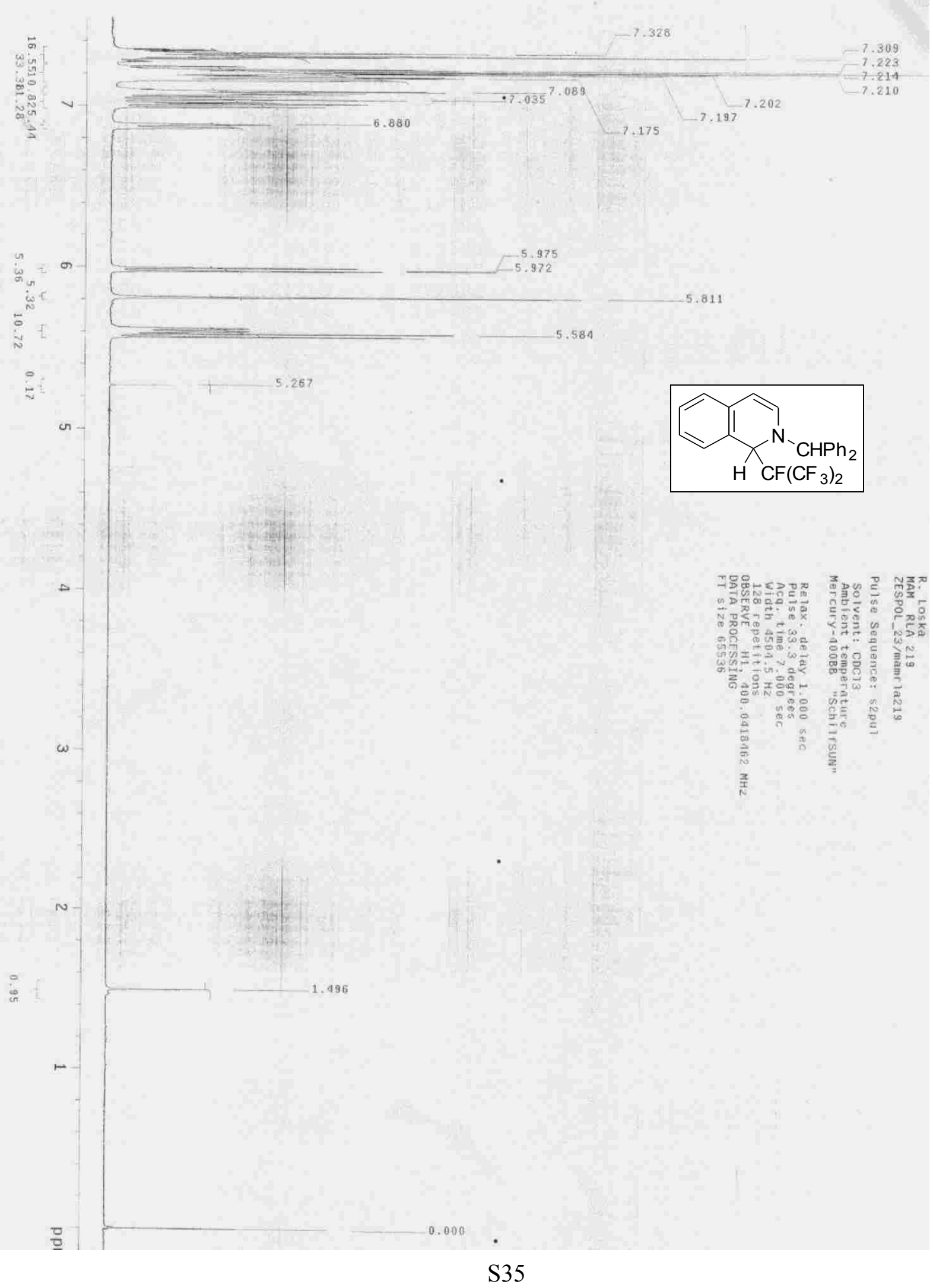




\section{Compound 2d: ${ }^{13} \mathrm{C}$ NMR}

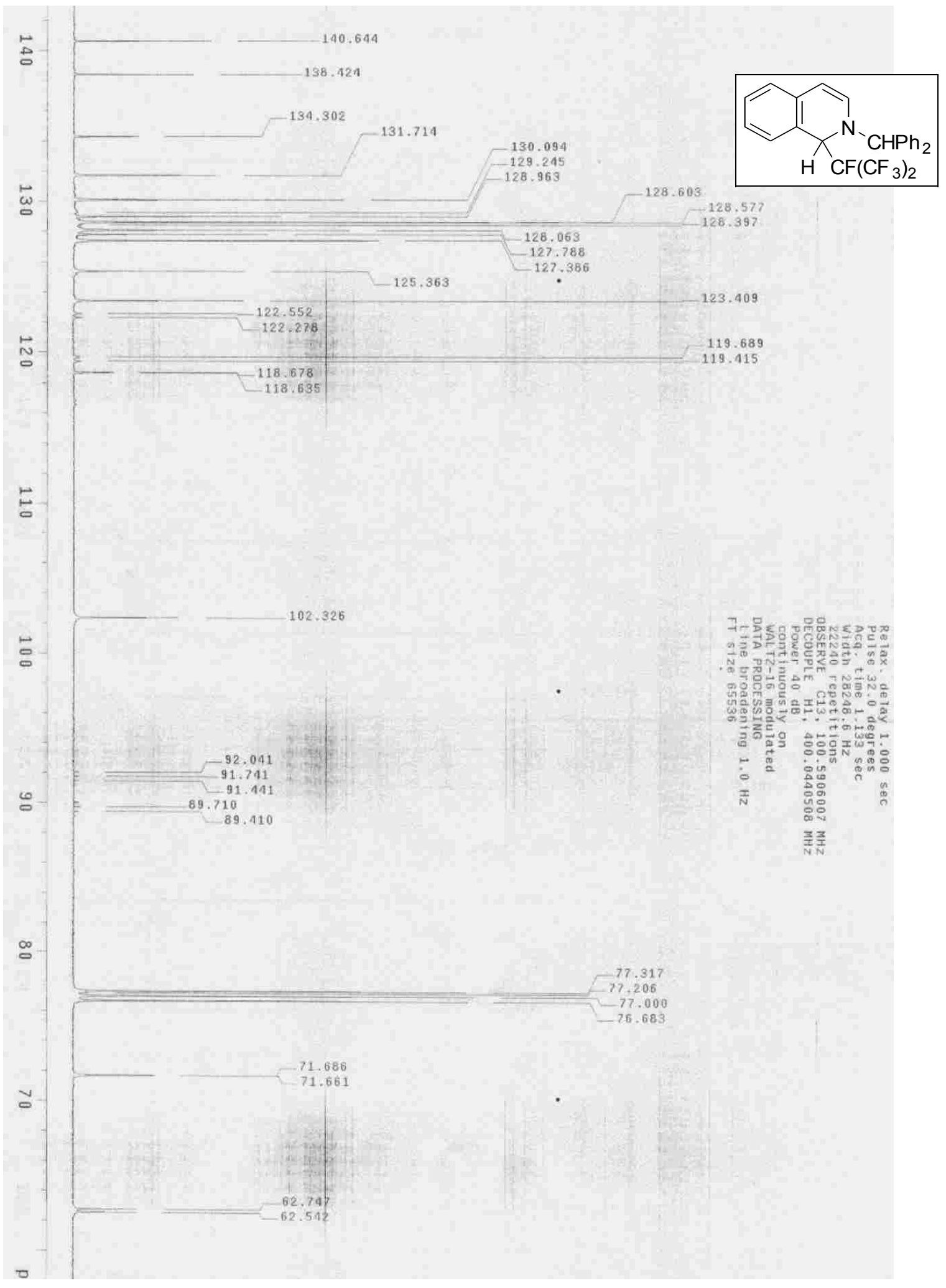




\section{Compound 2d: ${ }^{19} \mathrm{~F}$ NMR}

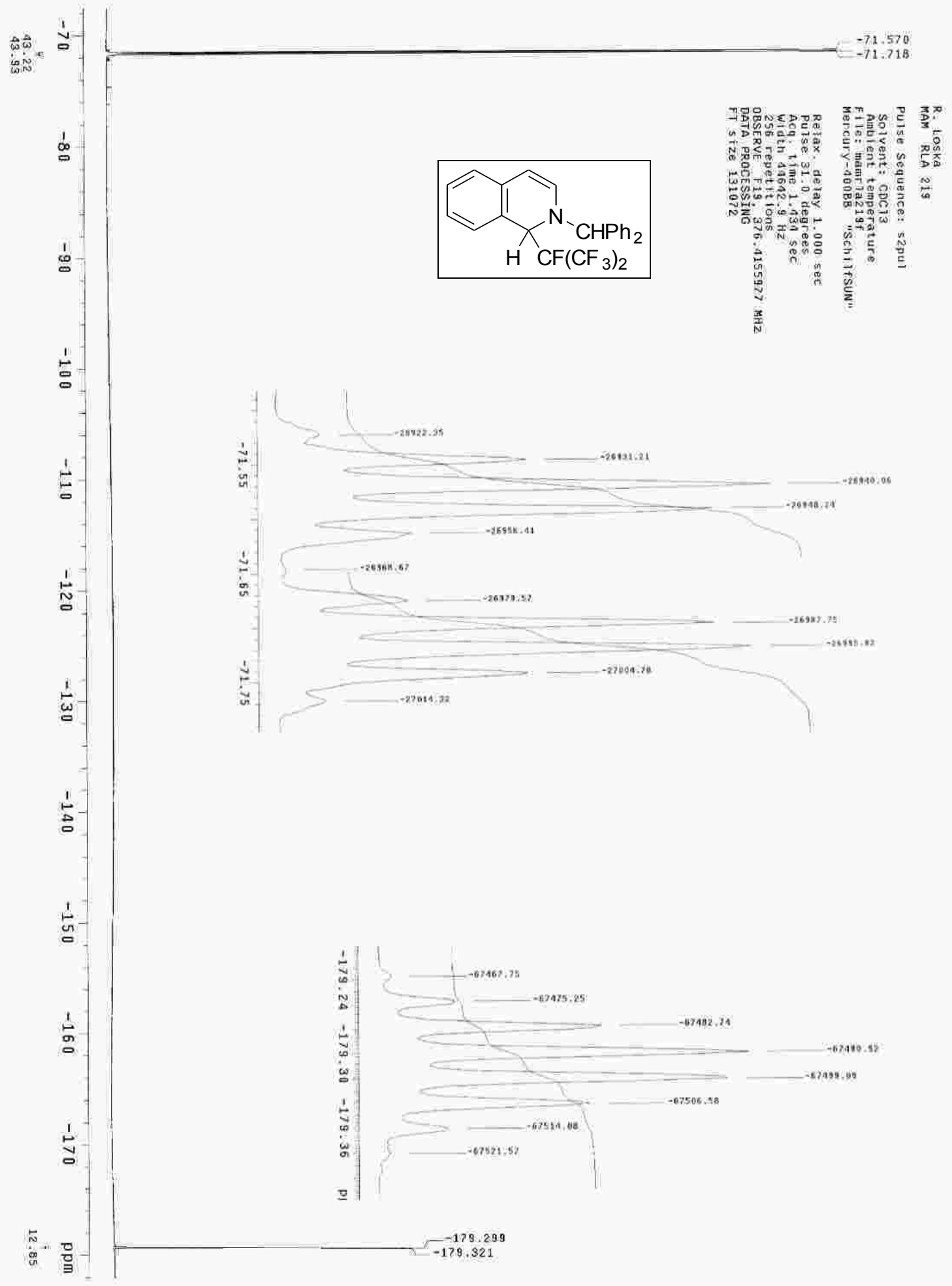




\section{Compound 2e: ${ }^{1} \mathrm{H}$ NMR}

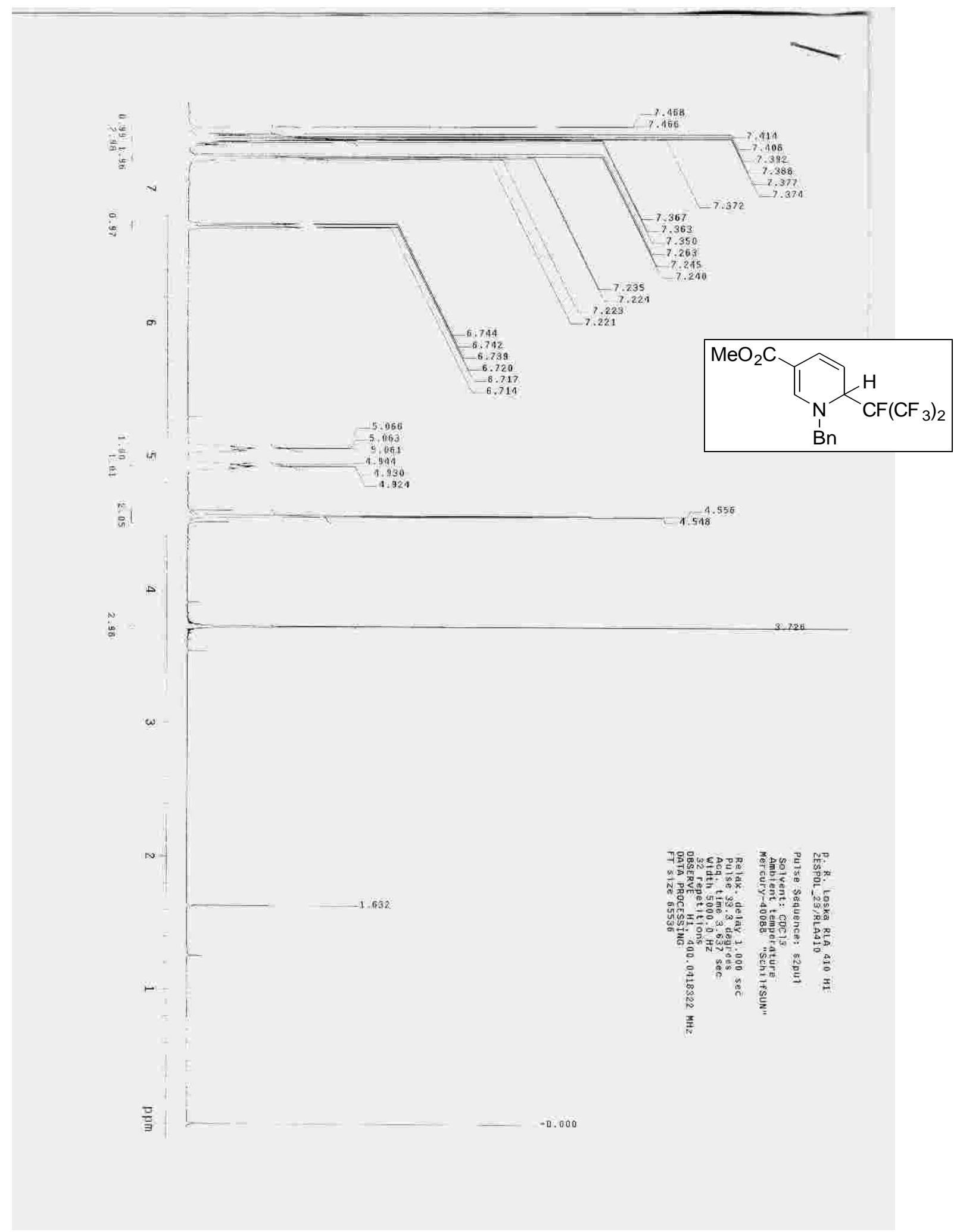




\section{Compound 2e: ${ }^{13} \mathrm{C}$ NMR}

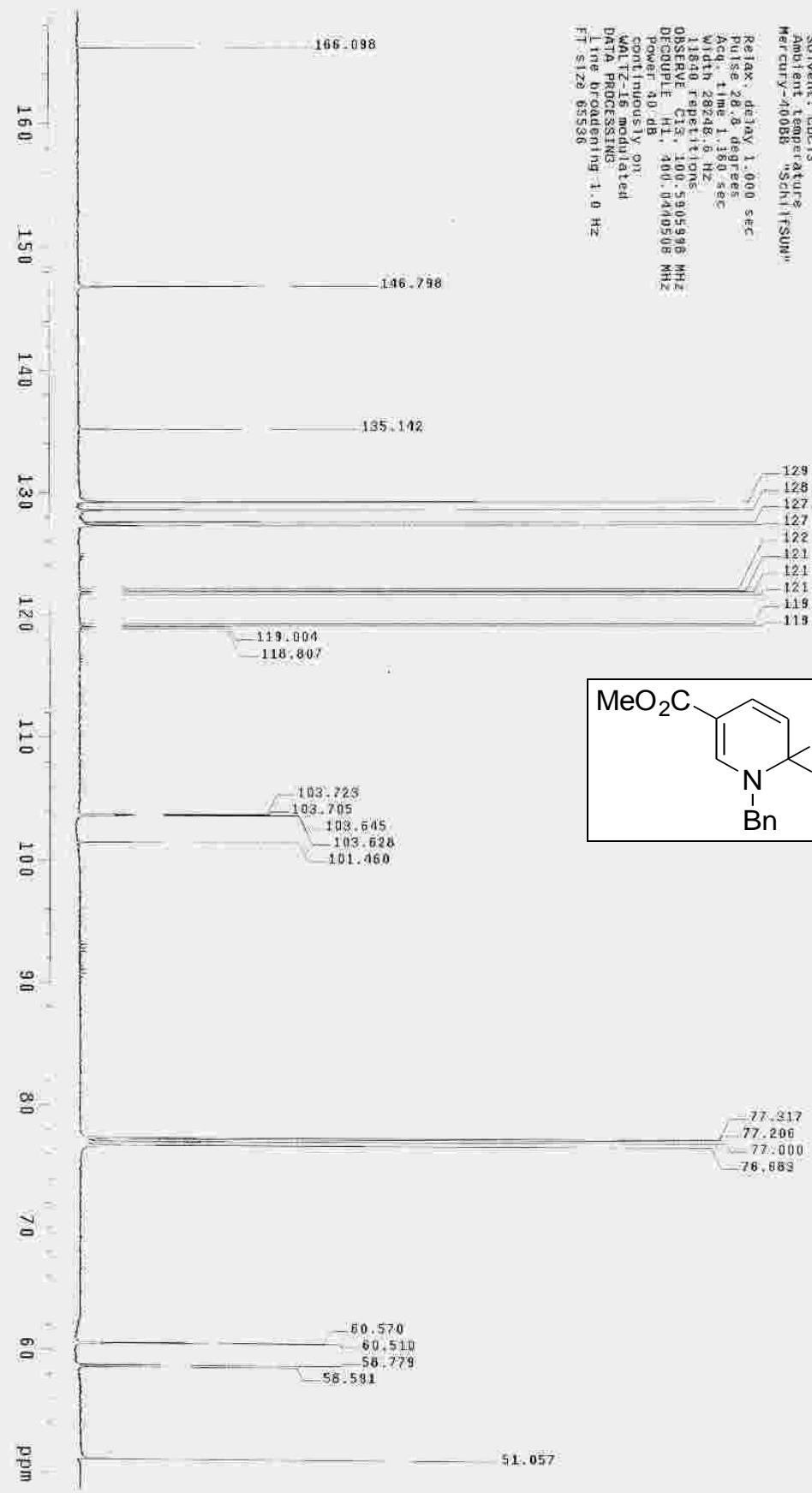




\section{Compound 2e: ${ }^{19}$ F NMR}

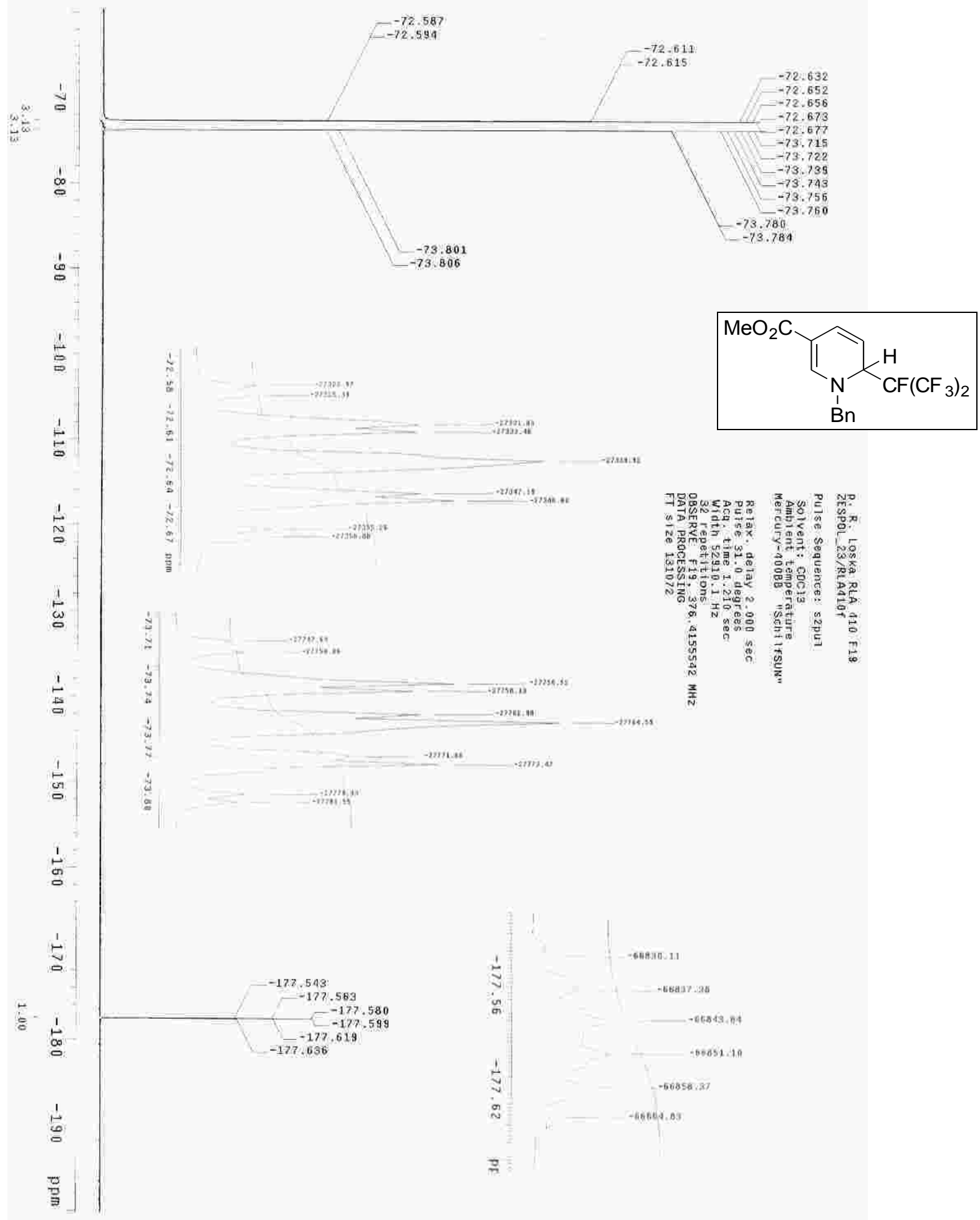




\section{Compound 3e: ${ }^{1} \mathrm{H}$ NMR}

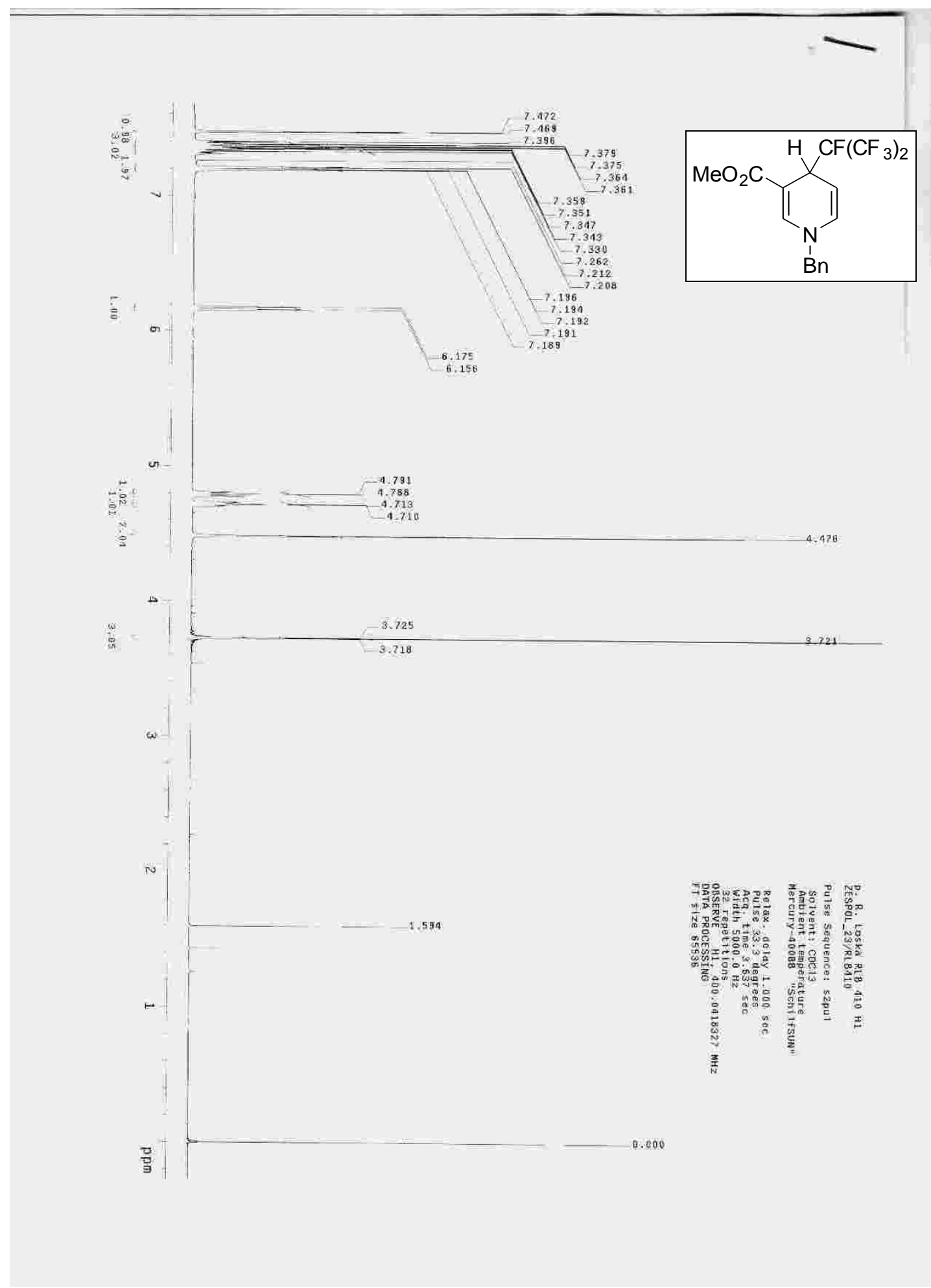


Compound 3e: ${ }^{13} \mathrm{C}$ NMR

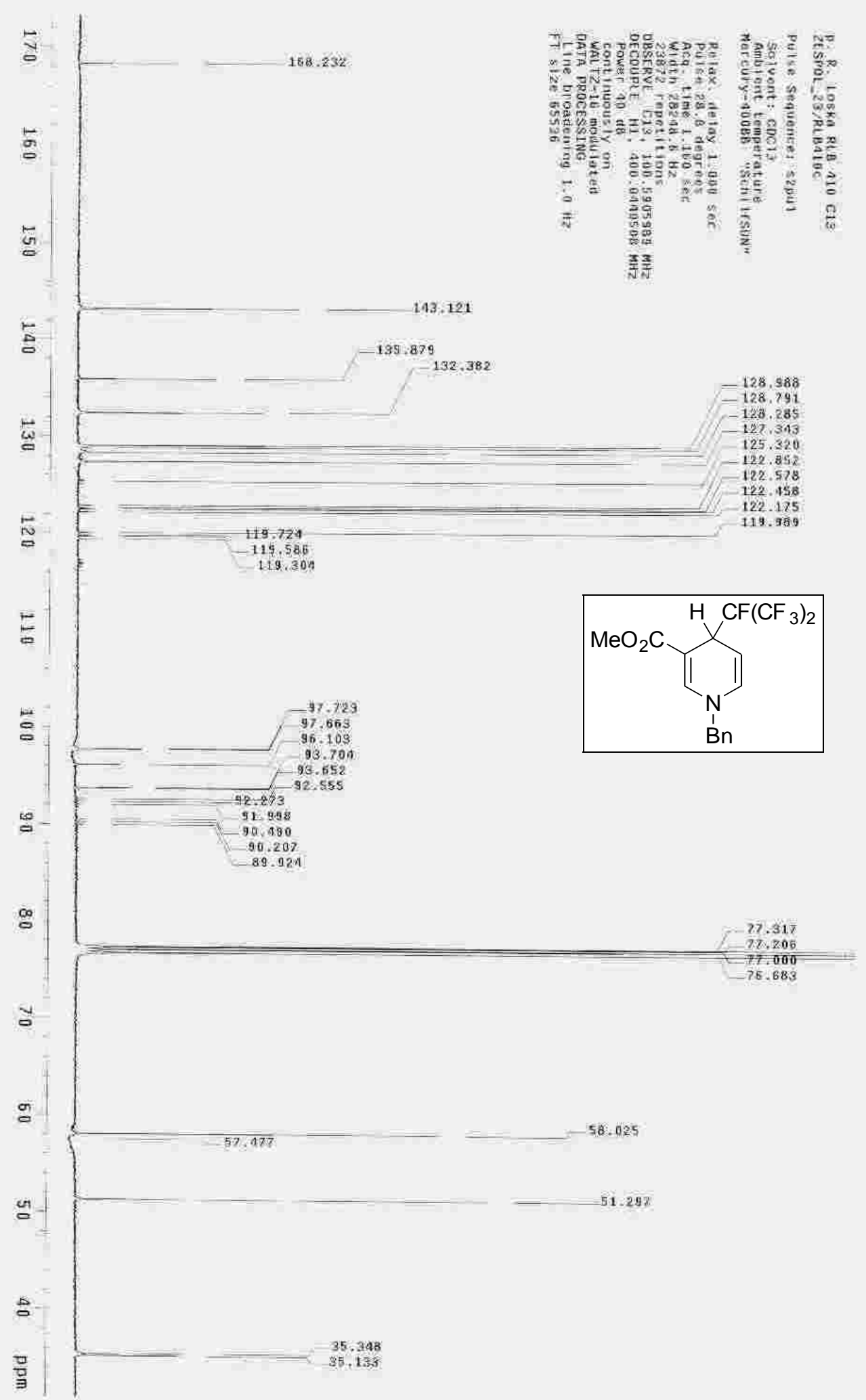


Compound 3e: ${ }^{19} \mathrm{~F}$ NMR

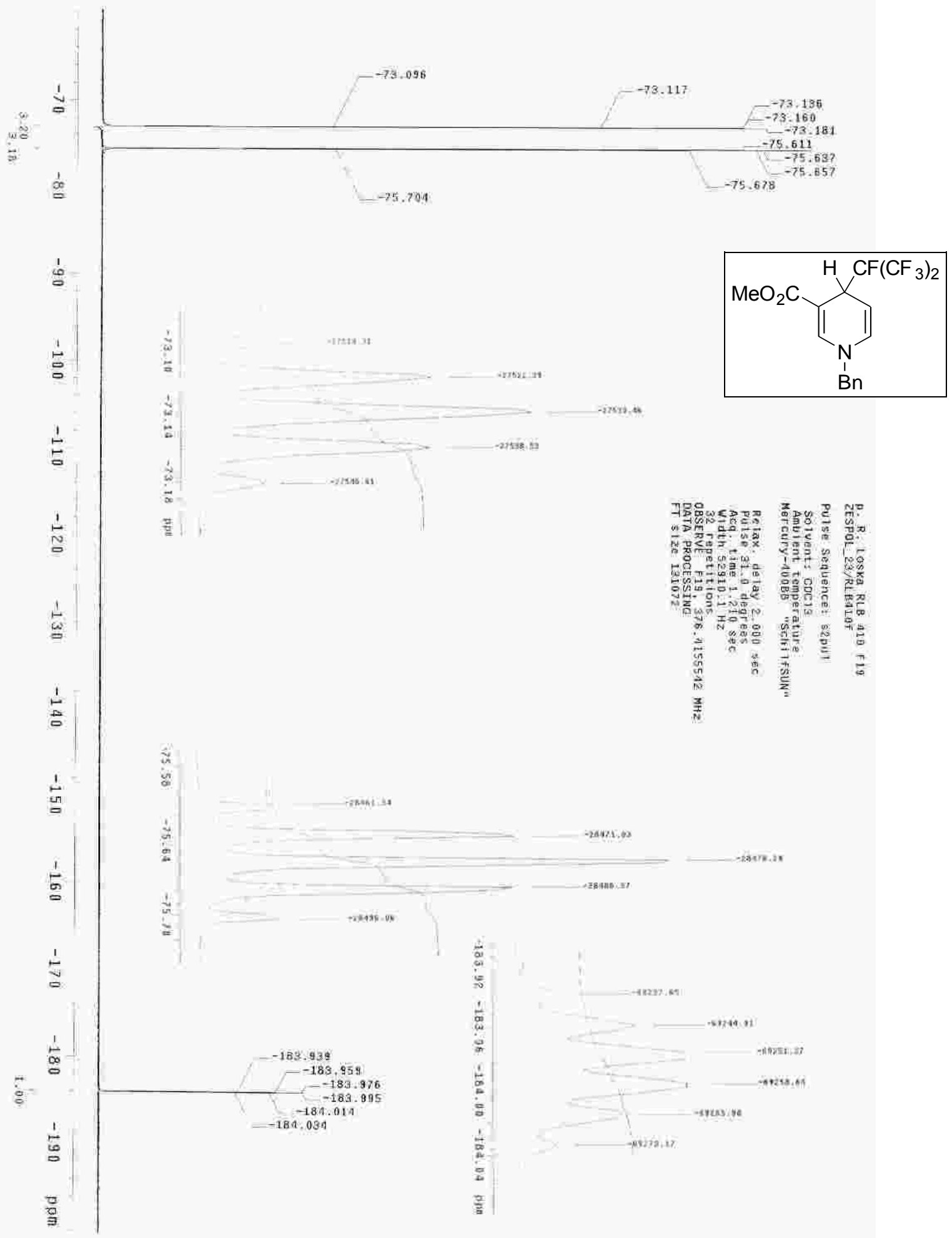




\section{Compound 2f: ${ }^{1} \mathrm{H}$ NMR}

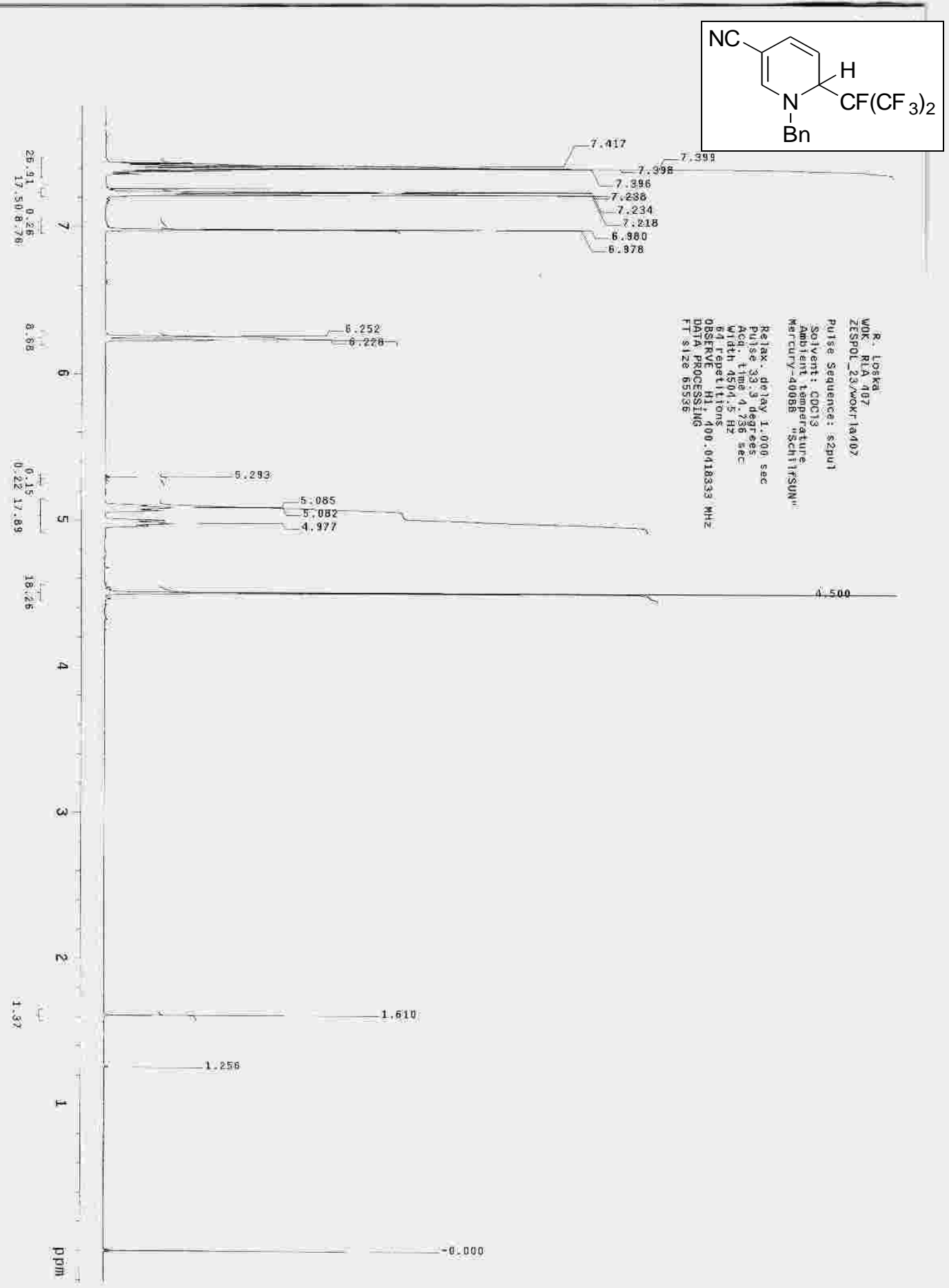




\section{Compound 2f: ${ }^{13} \mathrm{C}$ NMR}

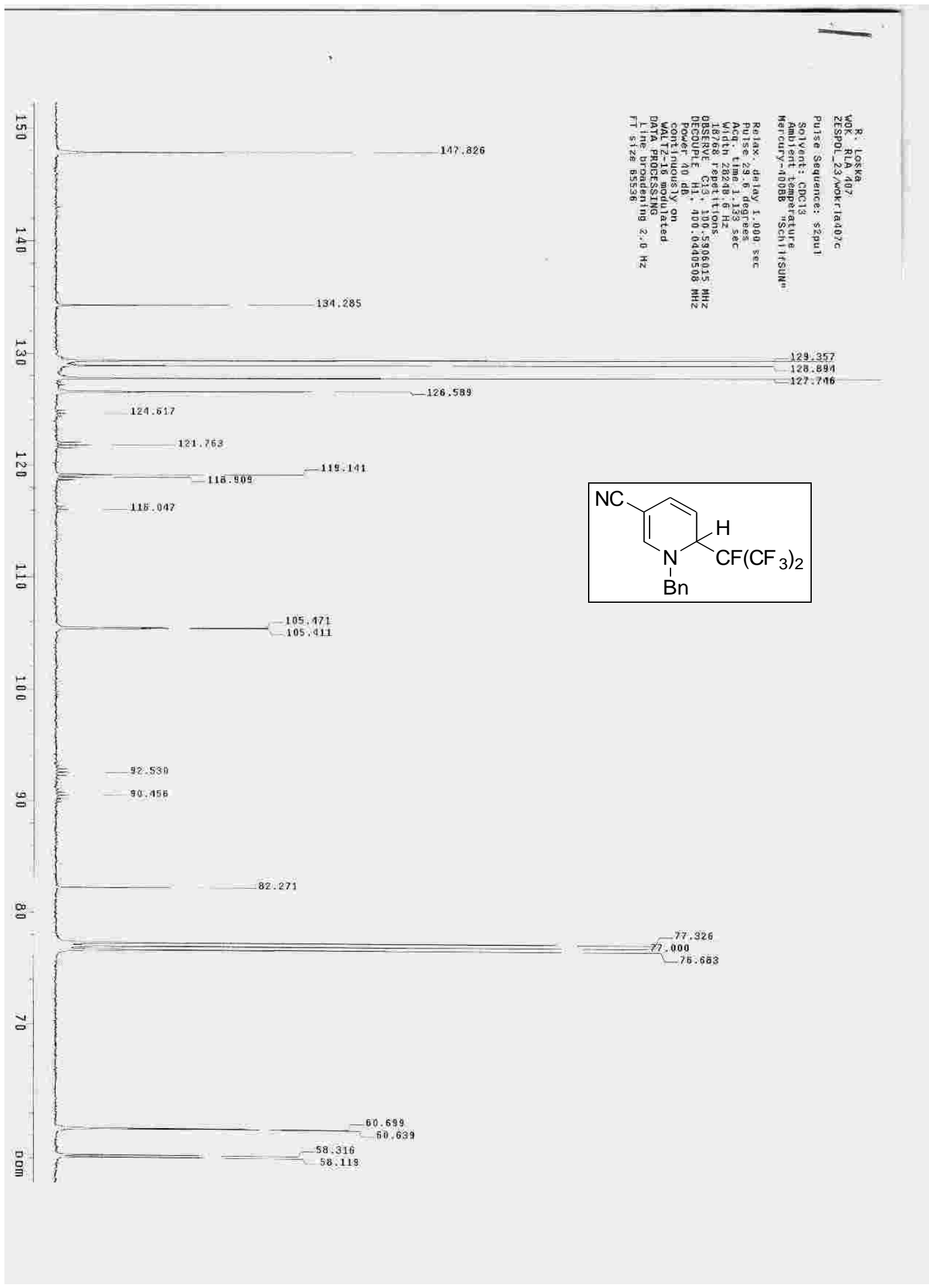




\section{Compound 2f: ${ }^{19}$ F NMR}

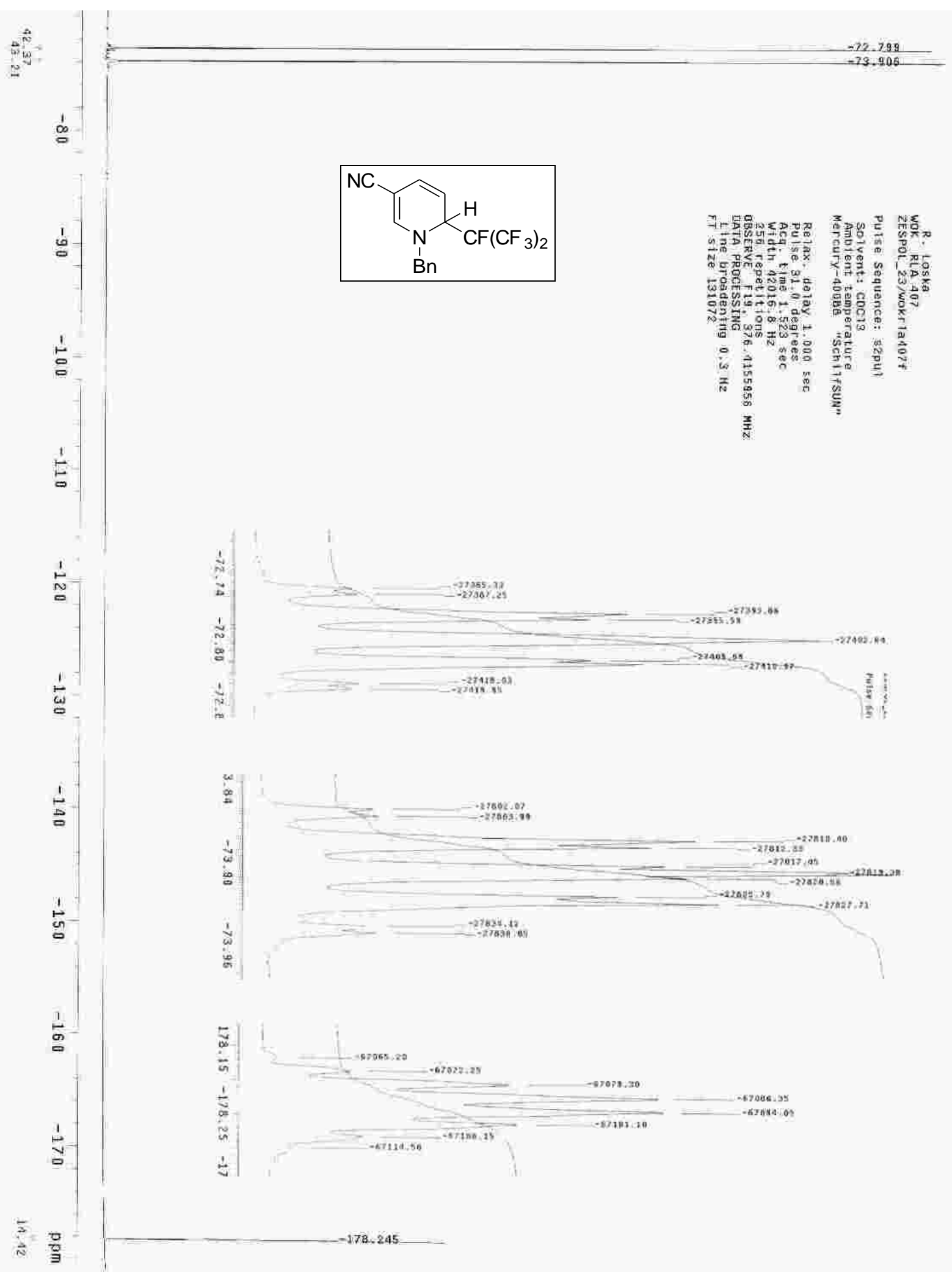




\section{Compound 3f: ${ }^{1} \mathrm{H}$ NMR}

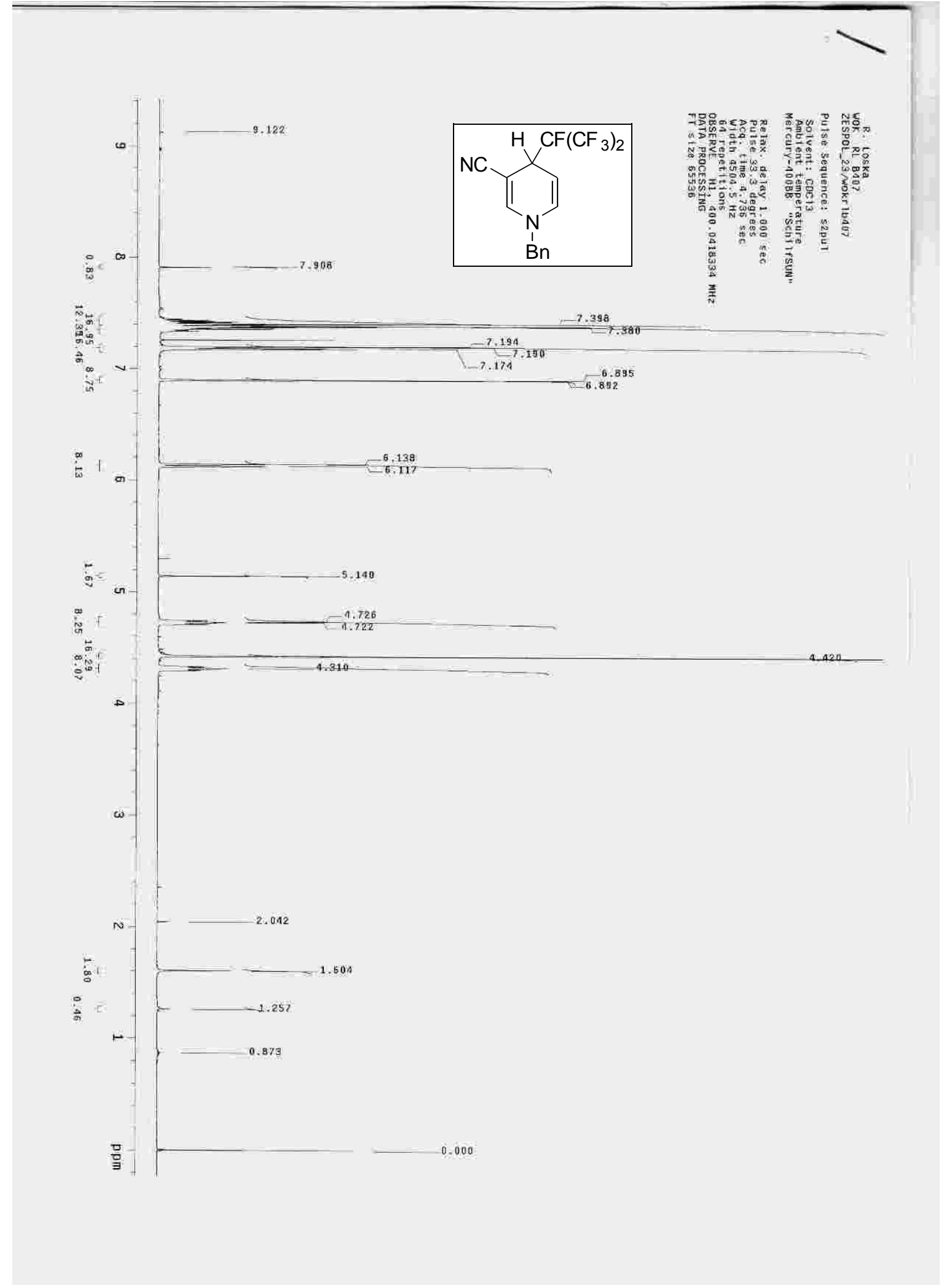




\section{Compound 3f: ${ }^{13} \mathrm{C}$ NMR}

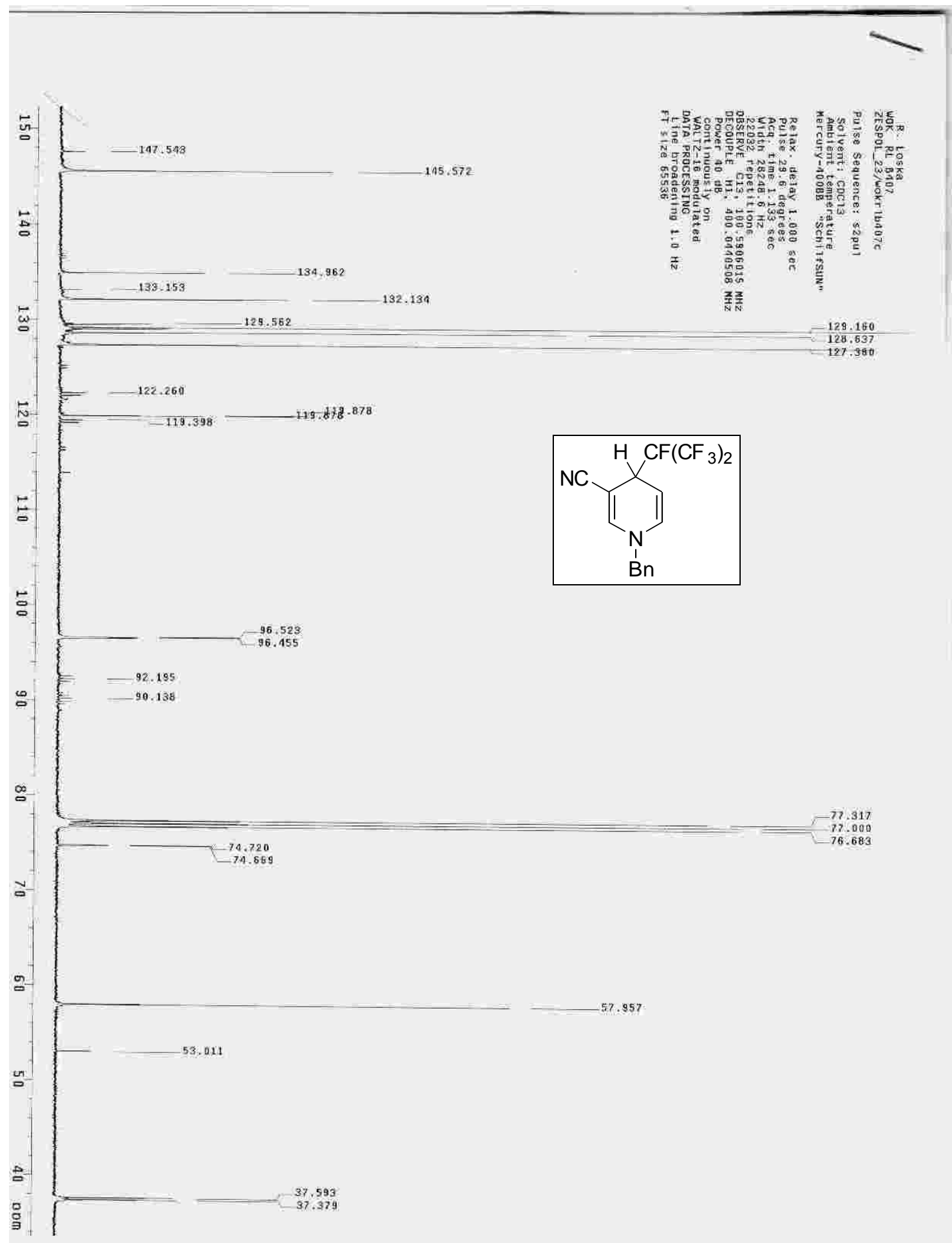




\section{Compound 3f: ${ }^{19}$ F NMR}

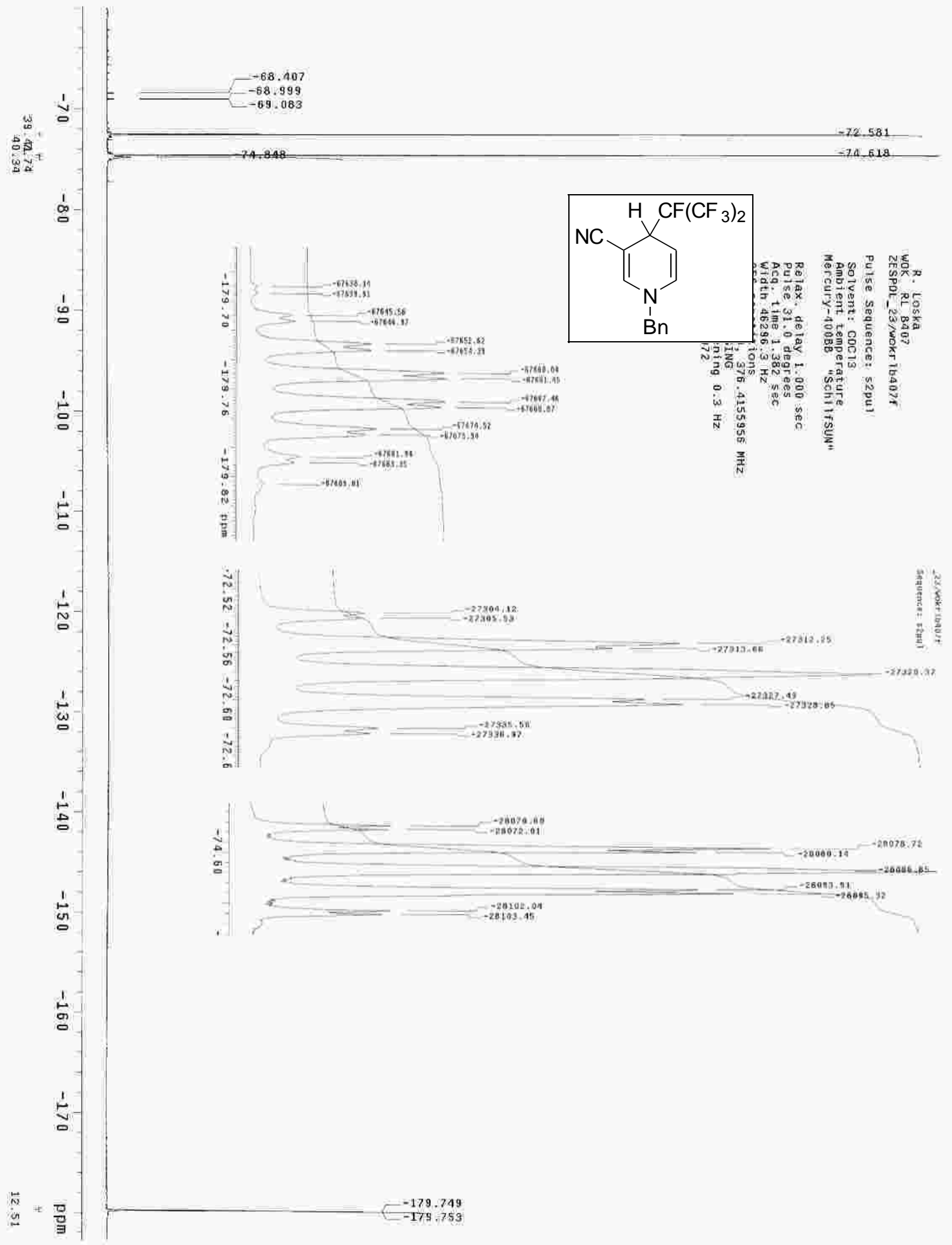




\section{Compound 2c': ${ }^{1} \mathrm{H}$ NMR}

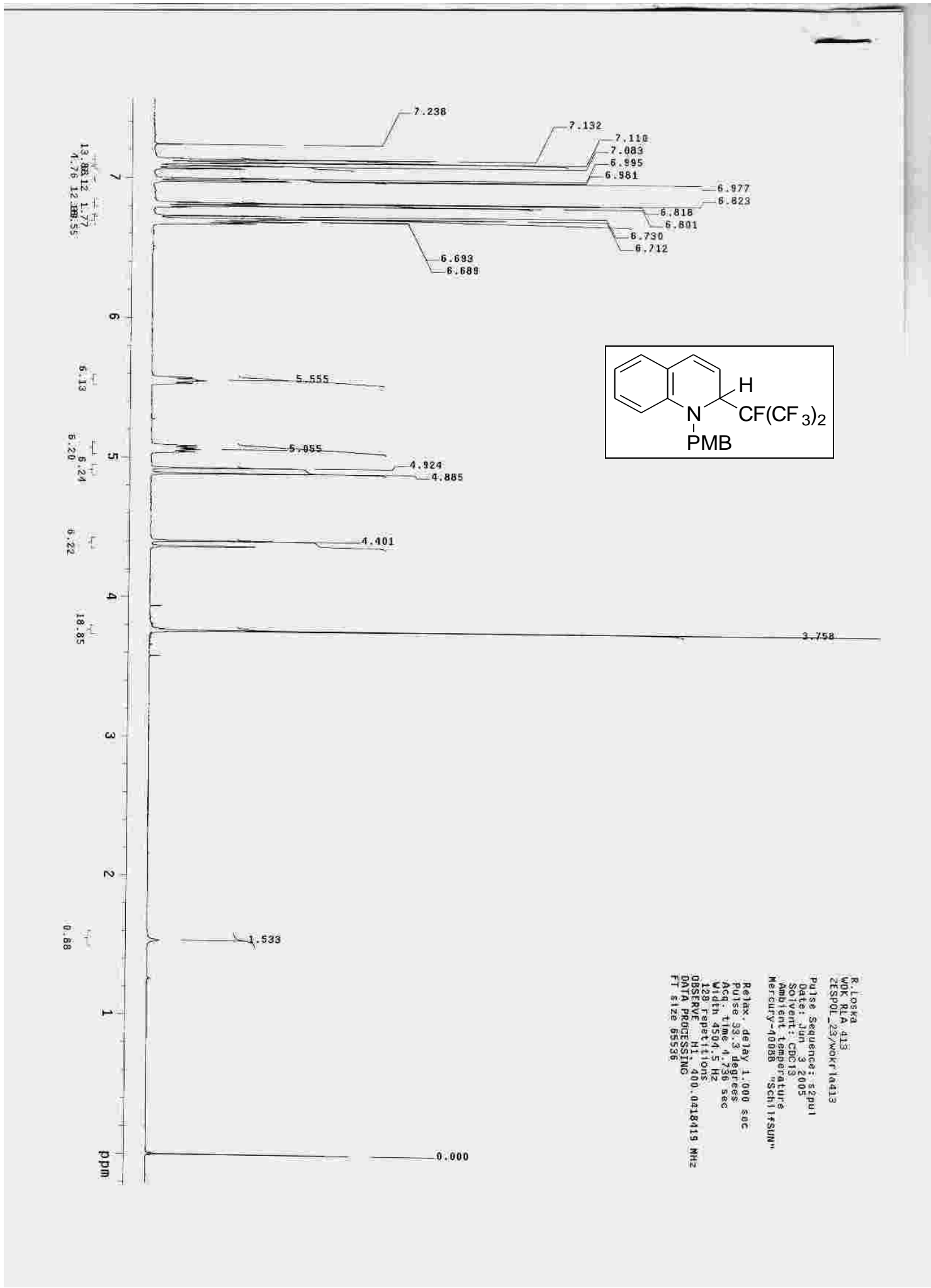




\section{Compound 2c': ${ }^{13} \mathrm{C}$ NMR}

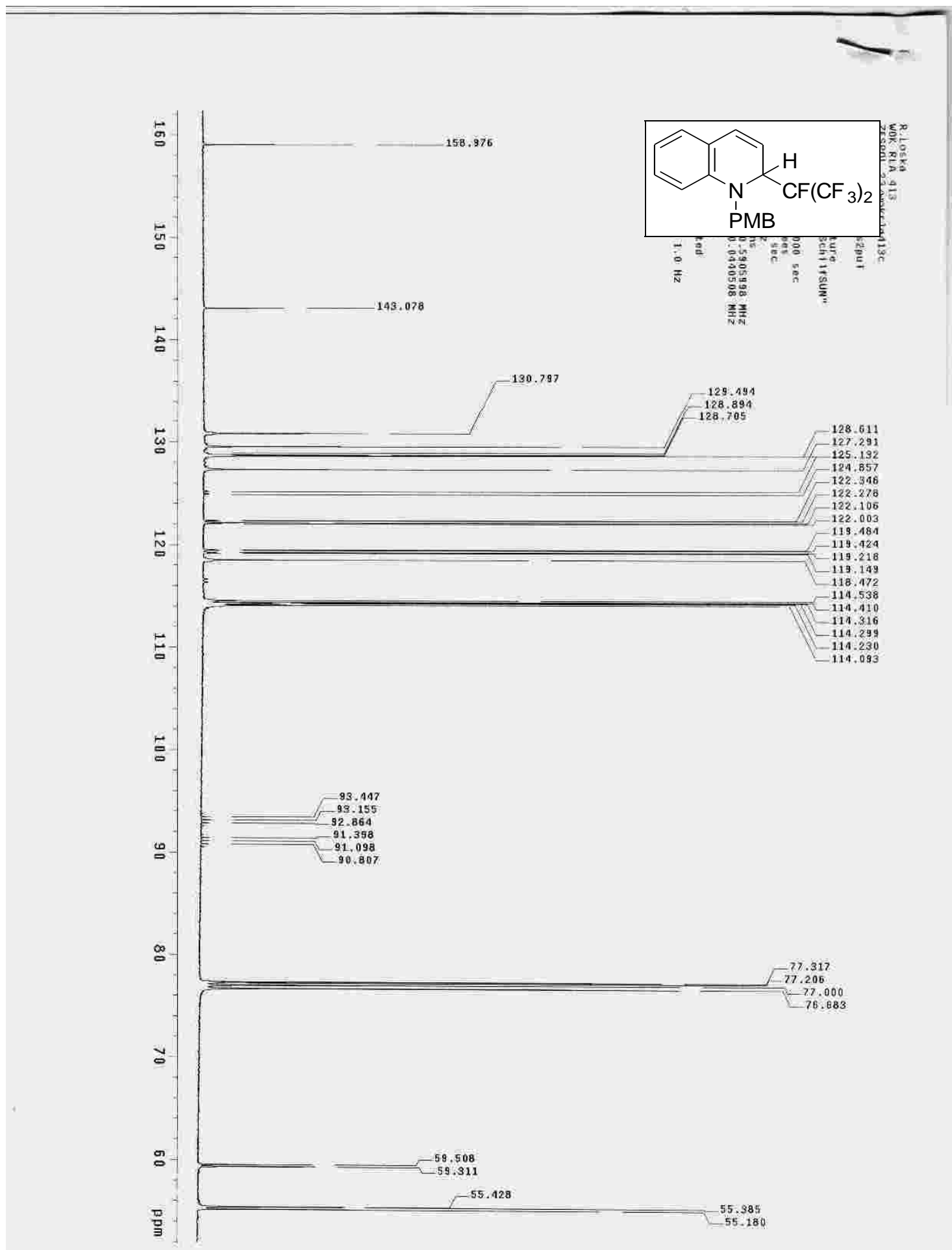


Compound 2c': ${ }^{19}$ F NMR

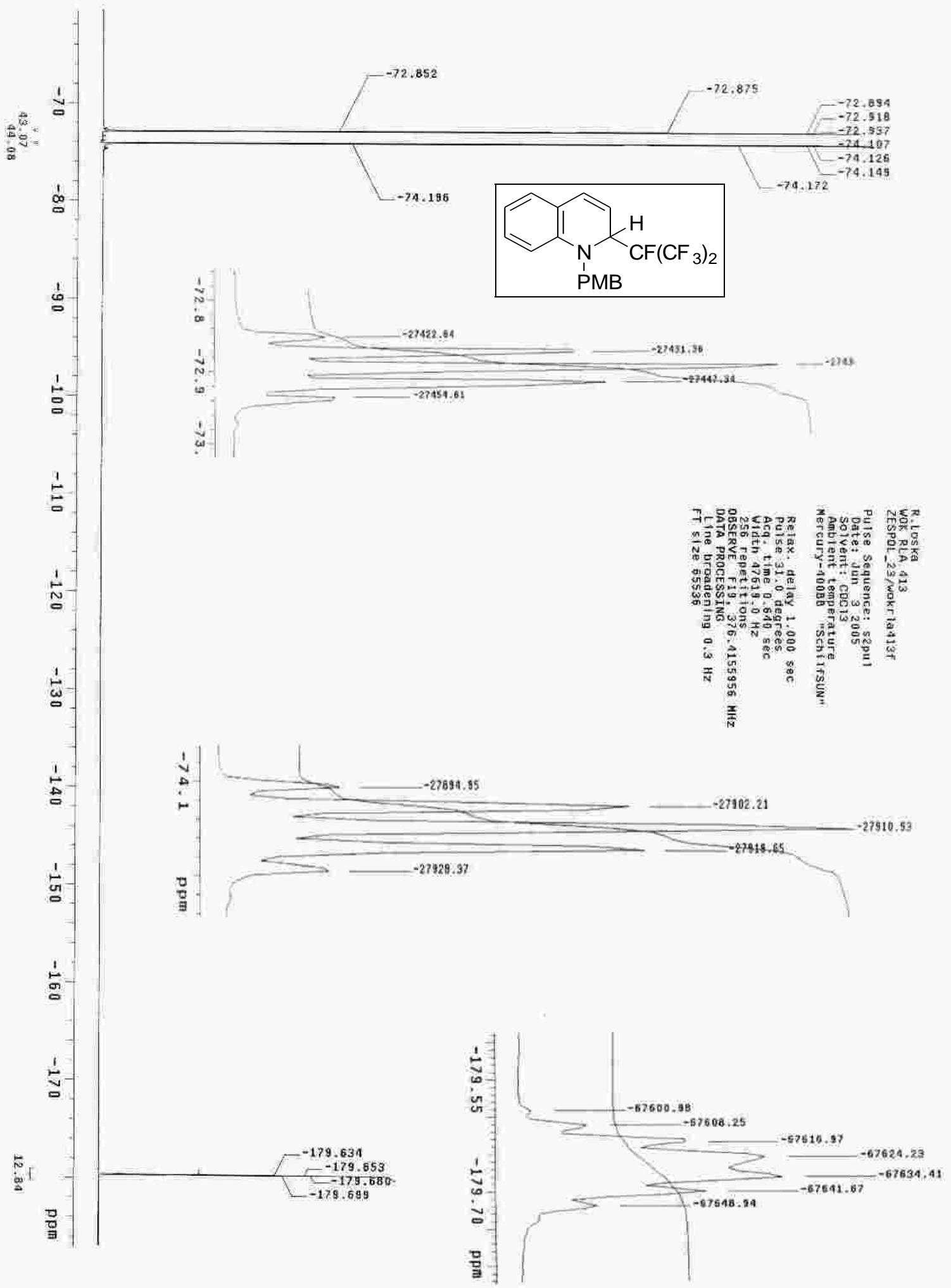




\section{Compound 2c': ${ }^{1} \mathrm{H}-{ }^{1} \mathrm{H}$ COSY}

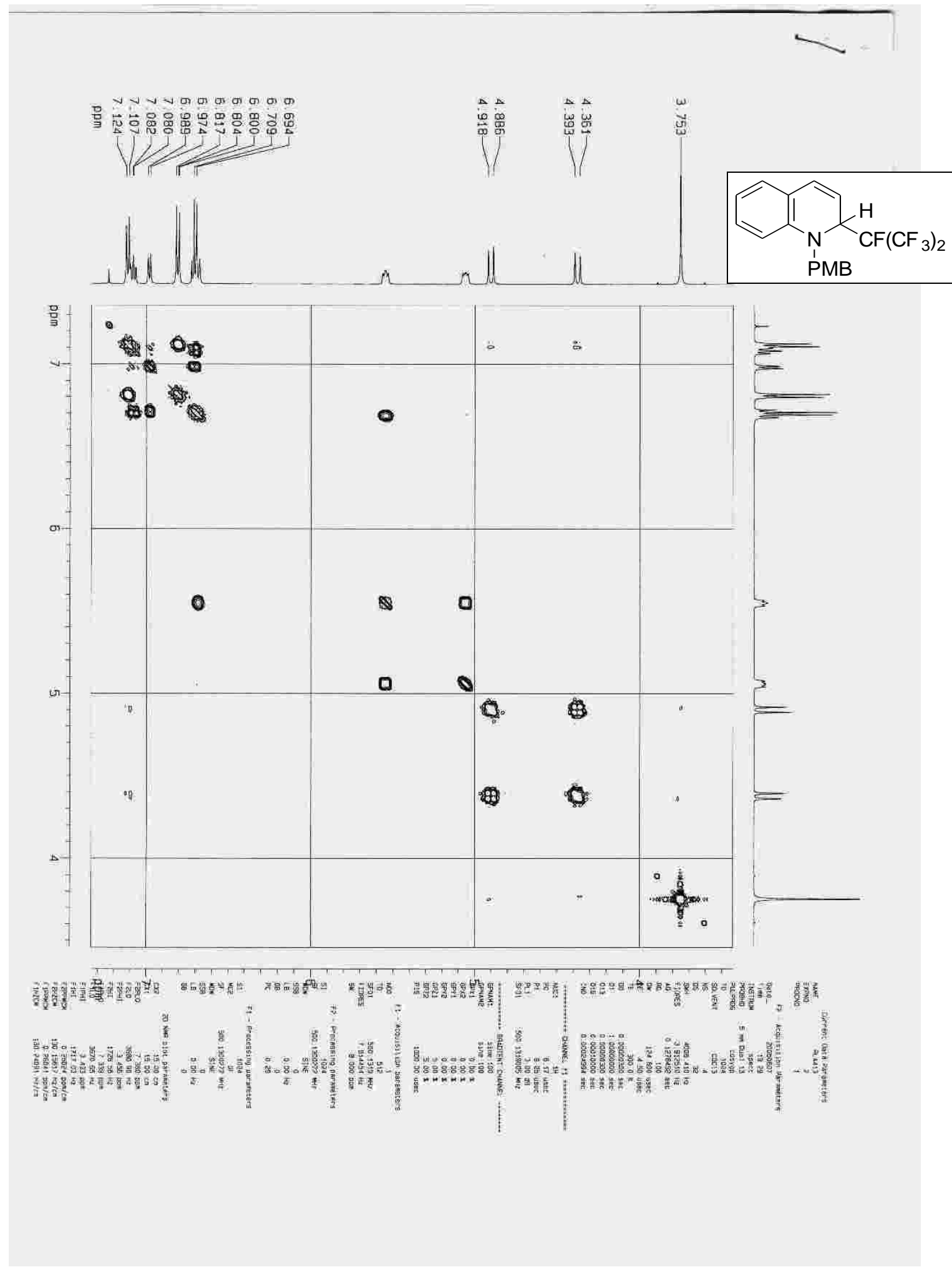




\section{Compound 2c': HSQC}

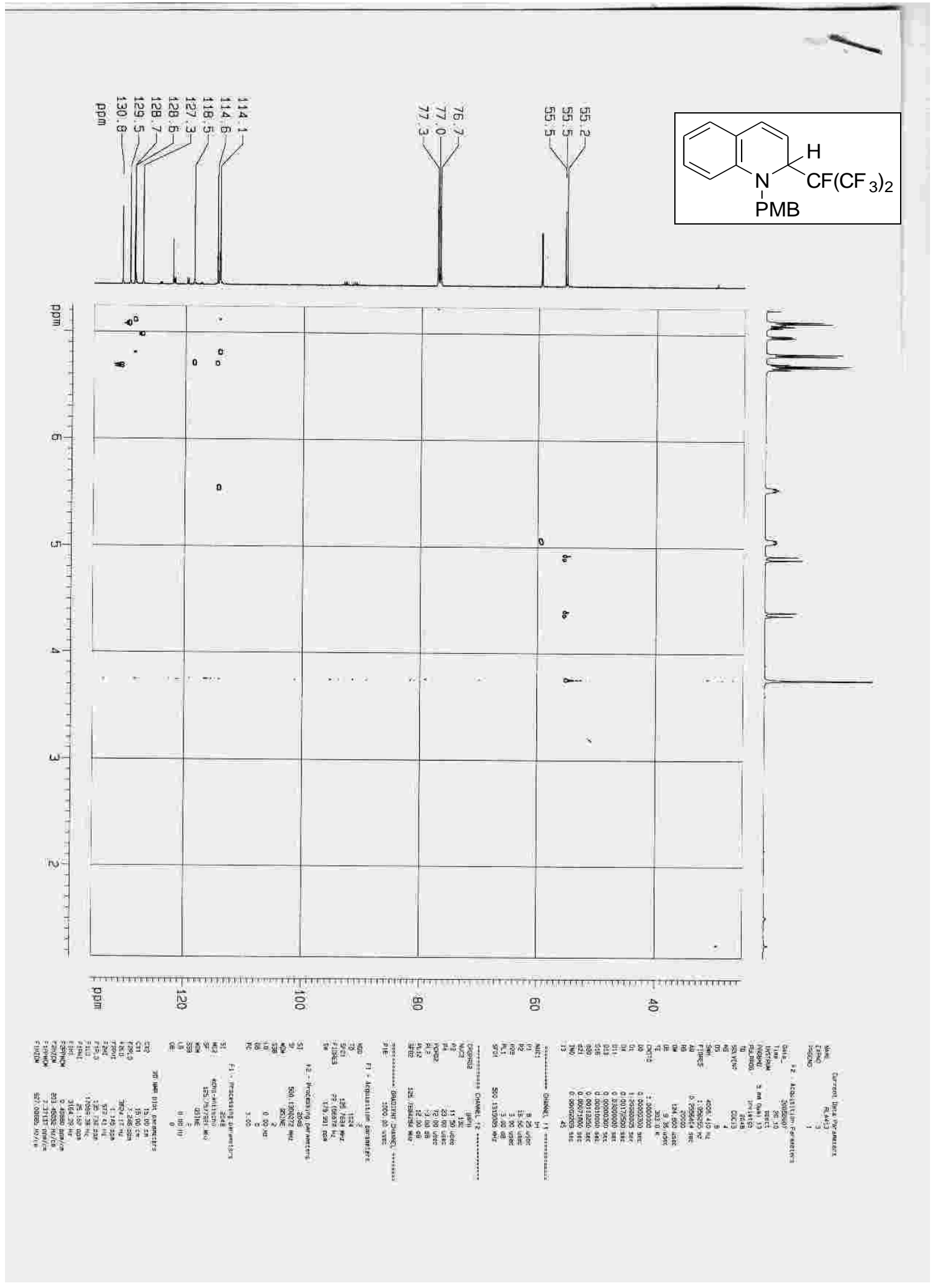




\section{Compound 2c': HMBC}

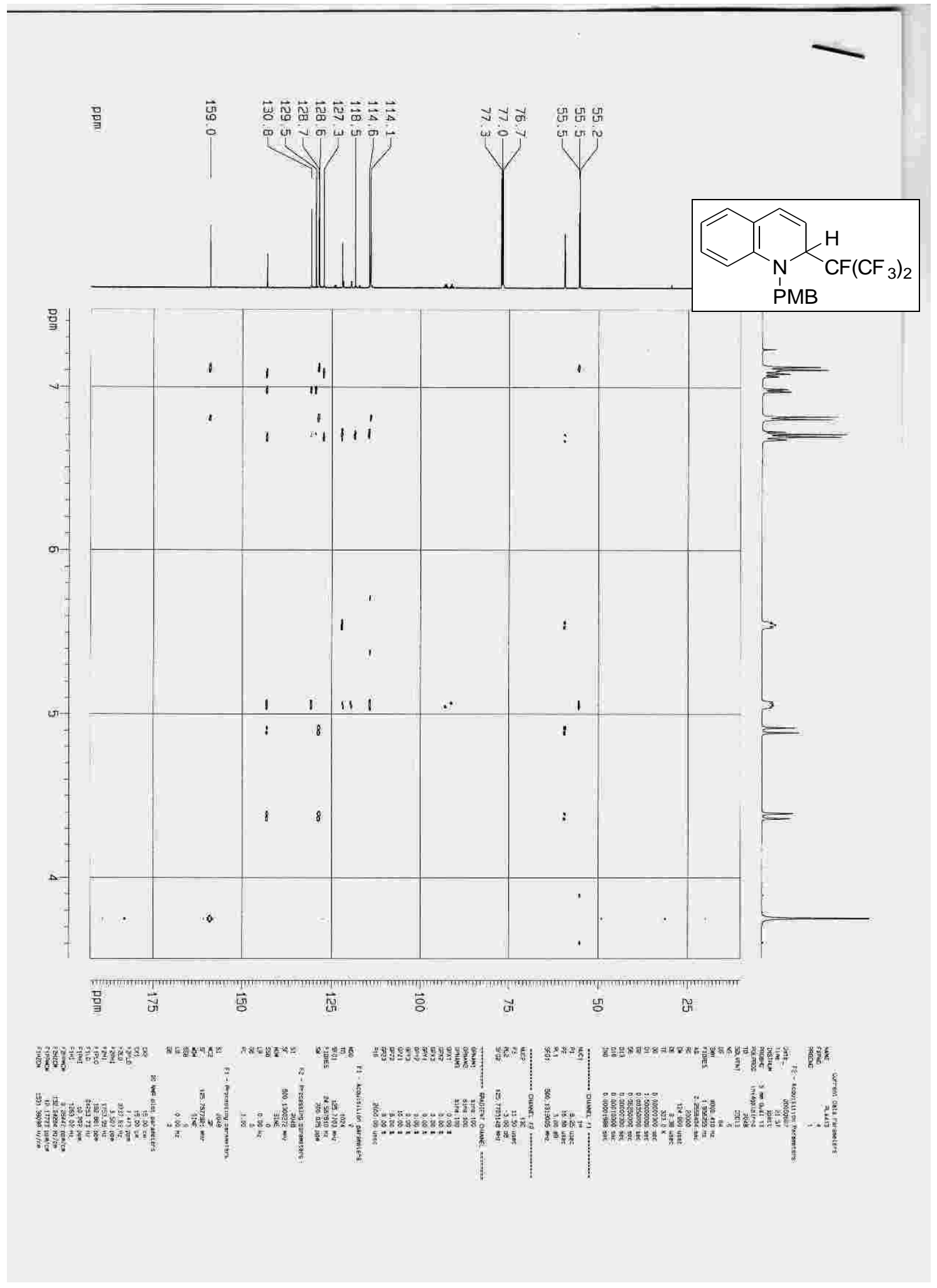




\section{Compound 3c': ${ }^{1} \mathrm{H}$ NMR}

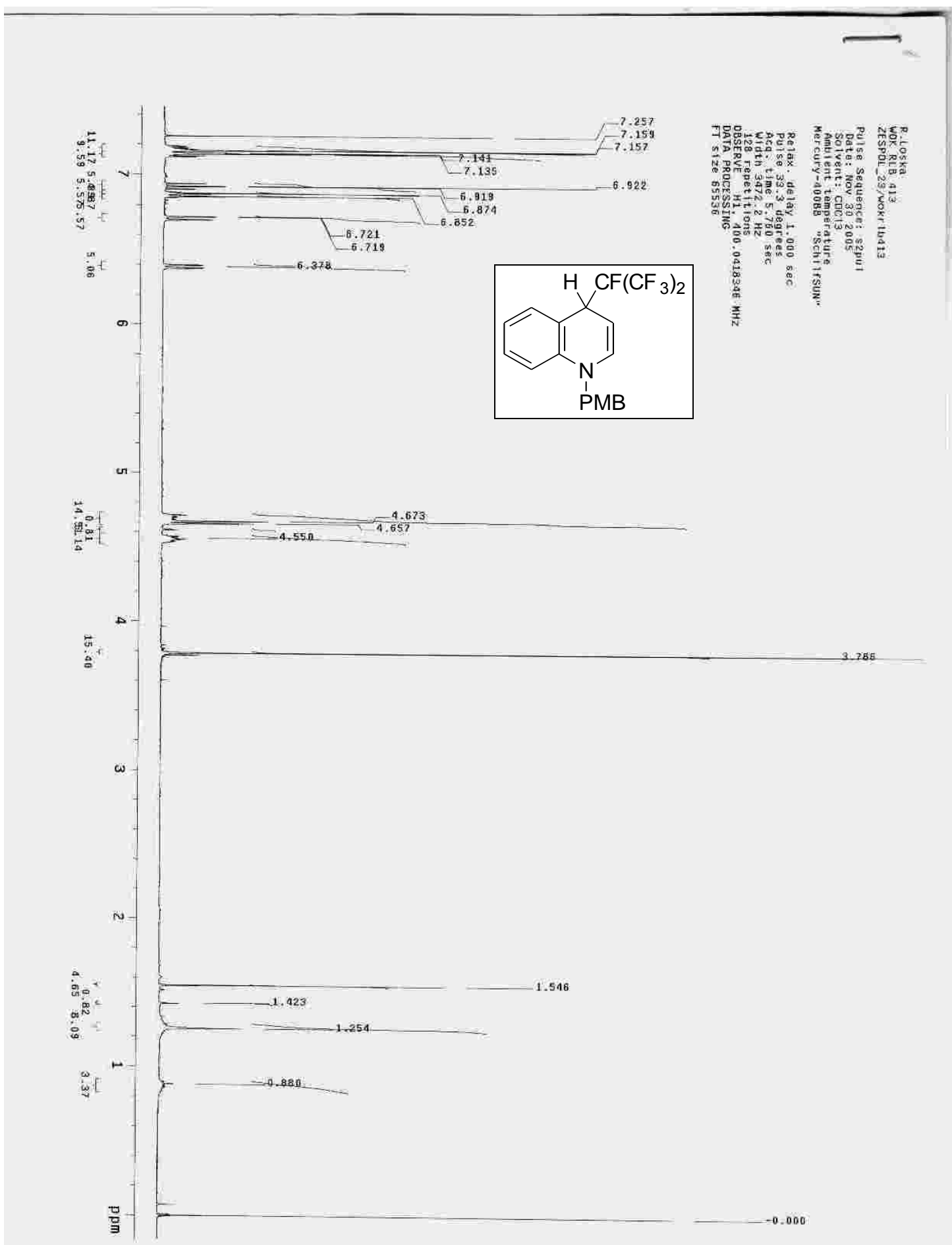




\section{Compound 3c': ${ }^{13} \mathrm{C}$ NMR}

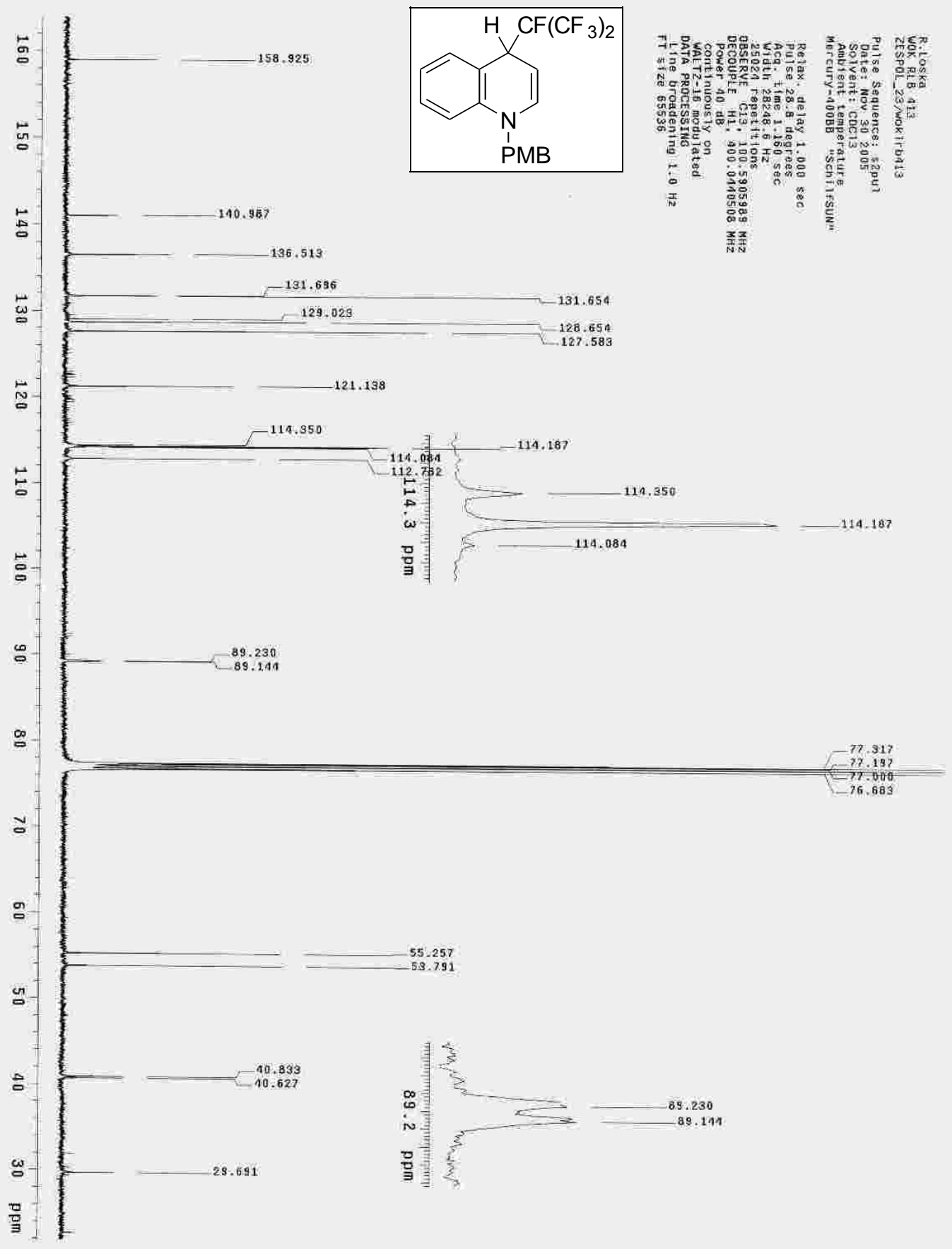


Compound 3c': ${ }^{19}$ F NMR
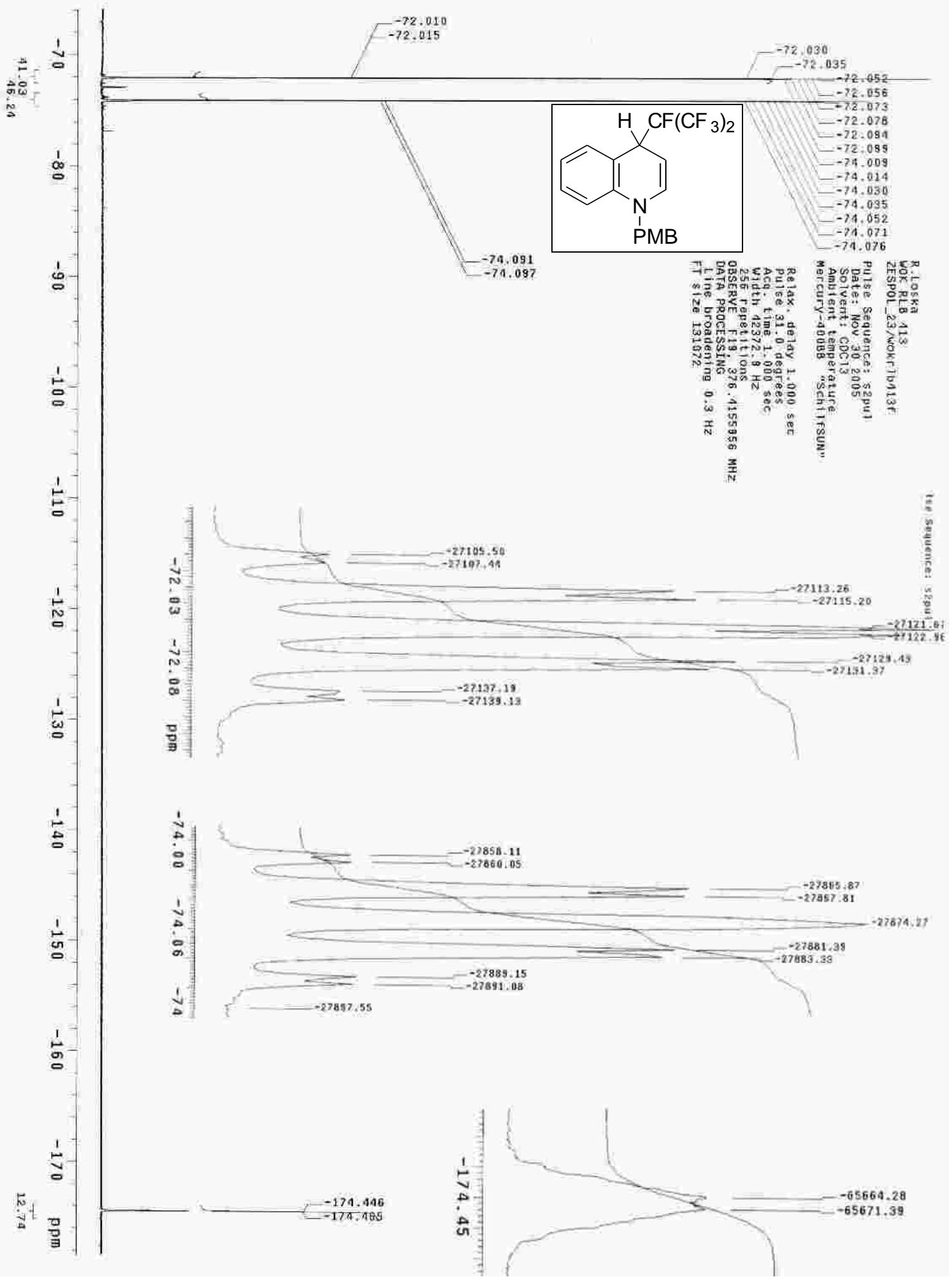
Compound 3c': ${ }^{1} \mathrm{H}-{ }^{1} \mathrm{H}$ COSY
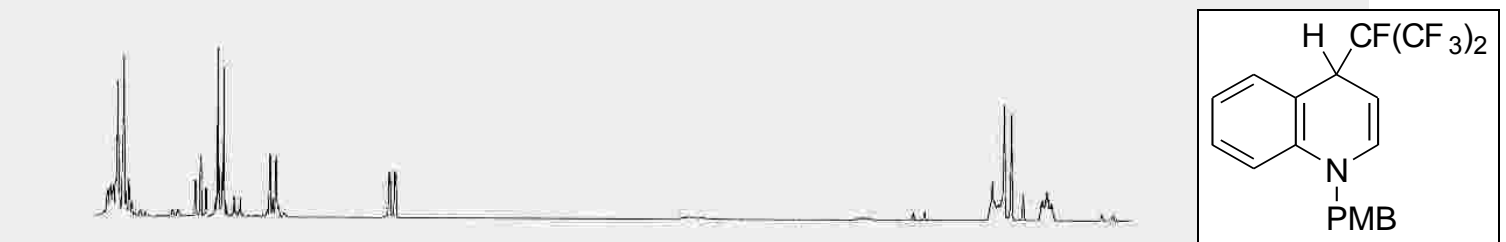

:
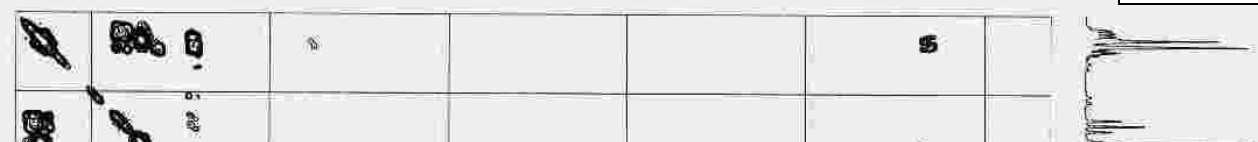

๓. : 5

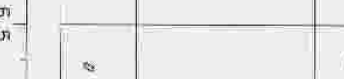

$-1$

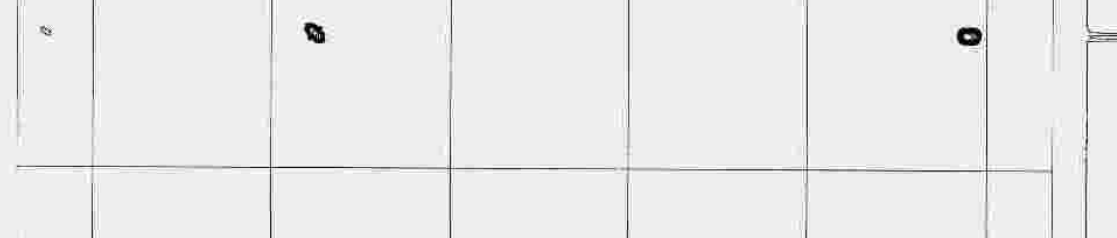

in
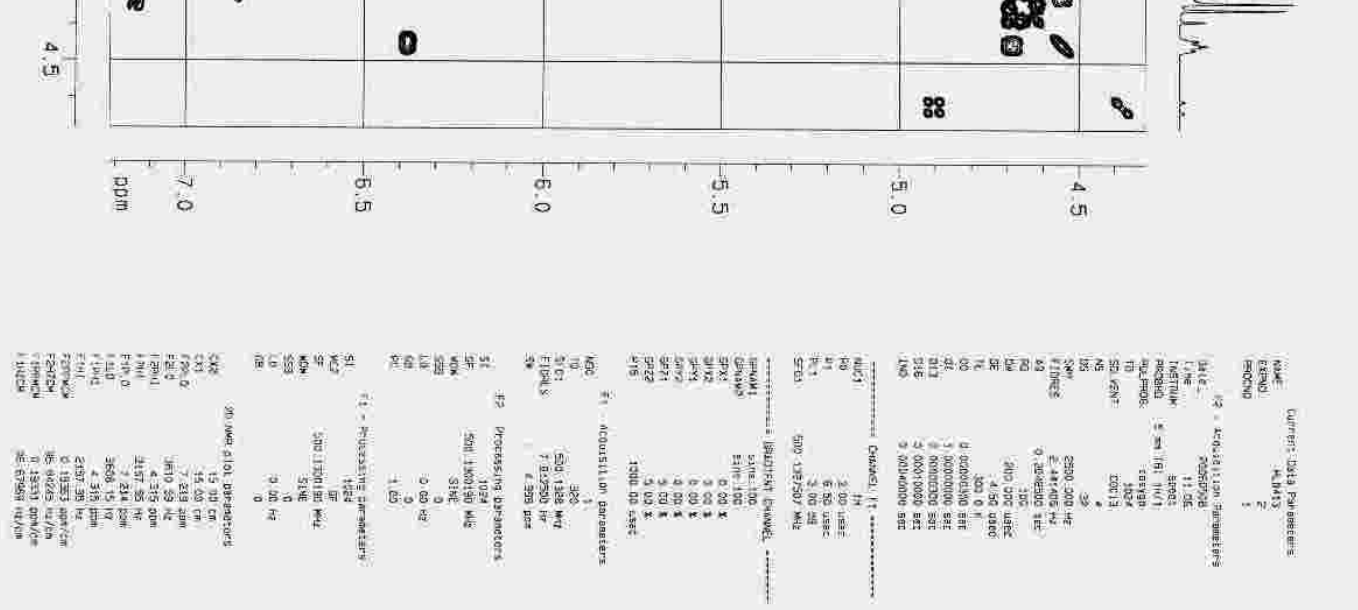


\section{Compound 2d': ${ }^{1} \mathrm{H}$ NMR}

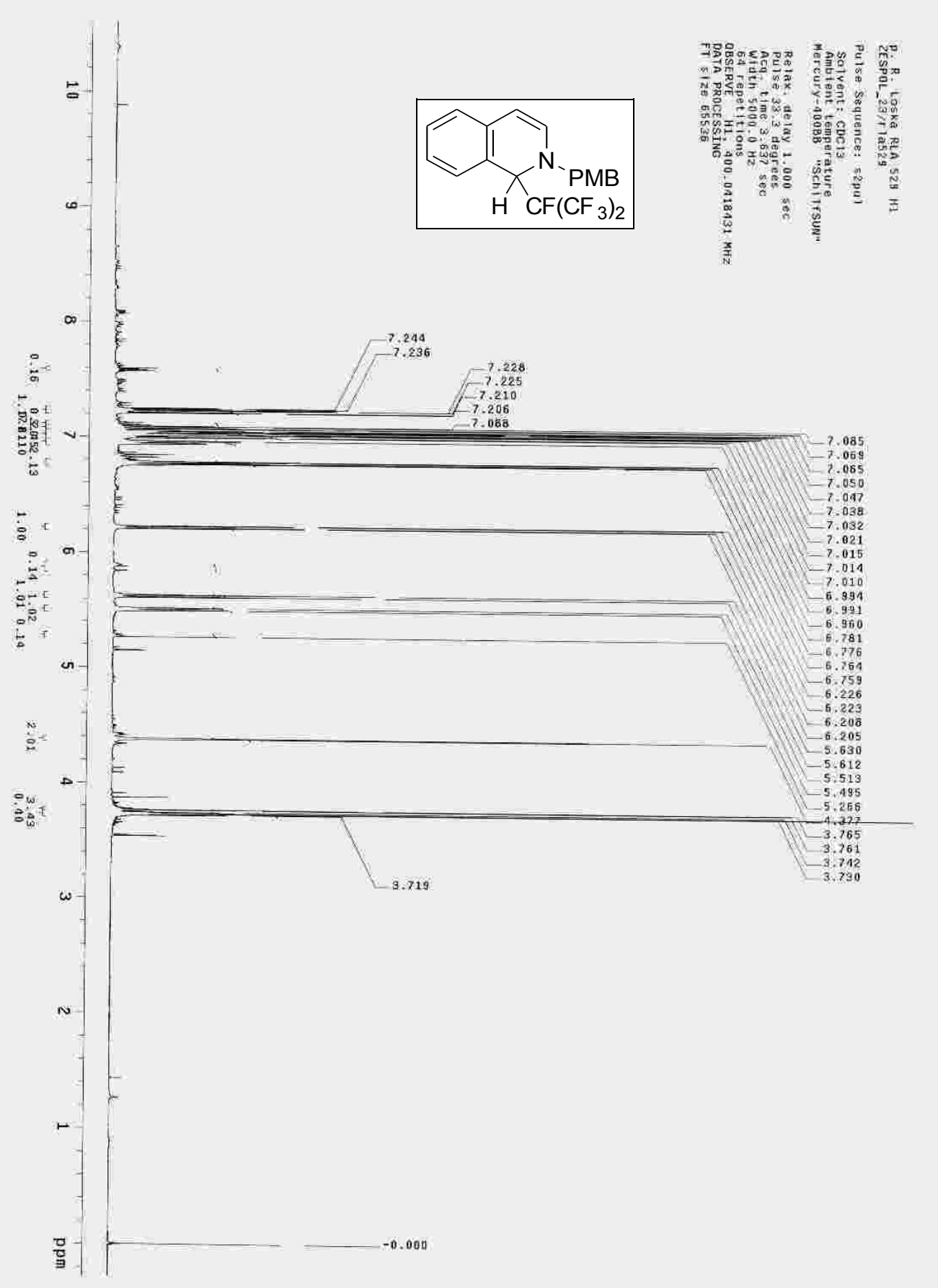


Compound 2d': ${ }^{13} \mathrm{C}$ NMR

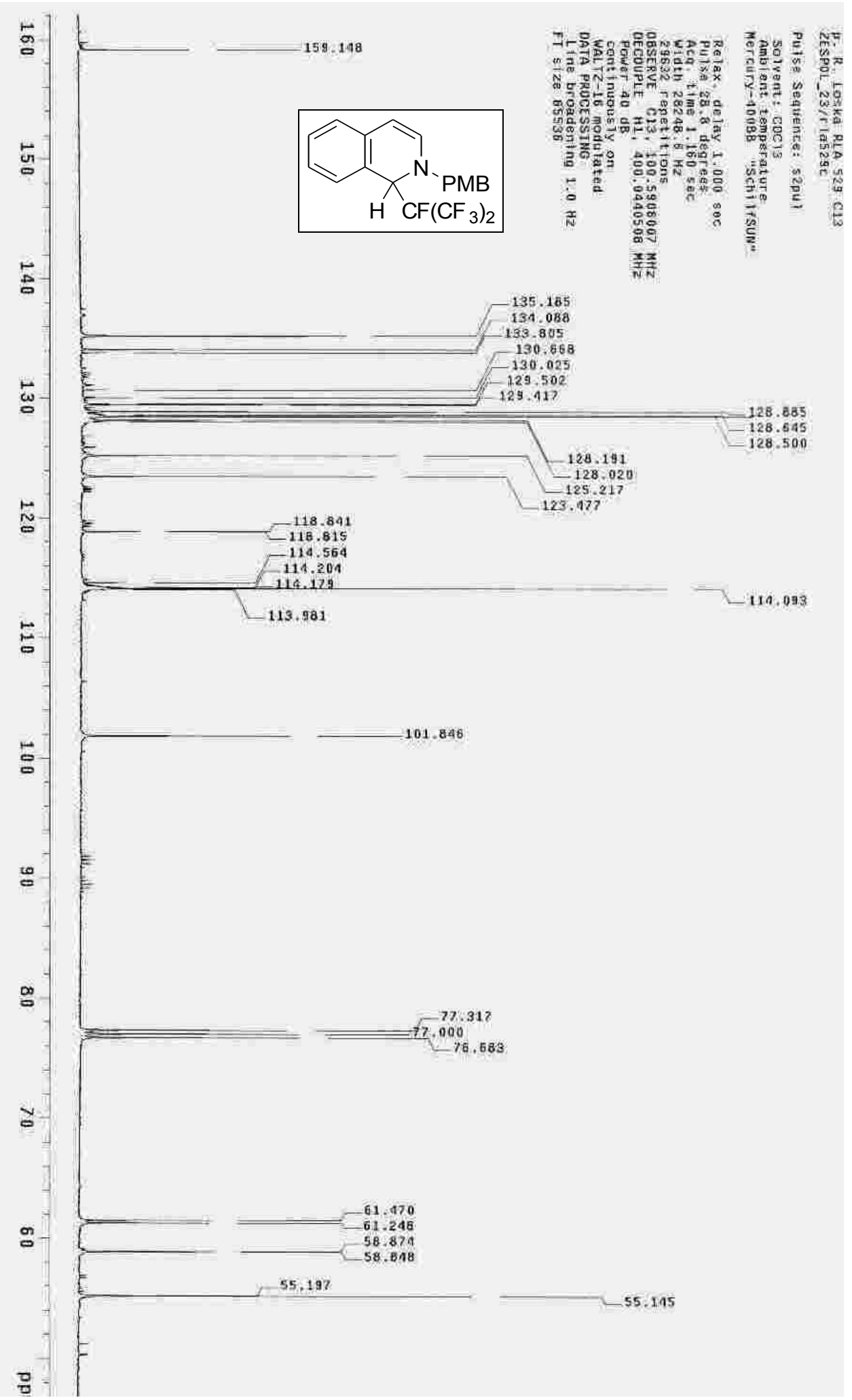




\section{Compound 2d': ${ }^{19}$ F NMR}

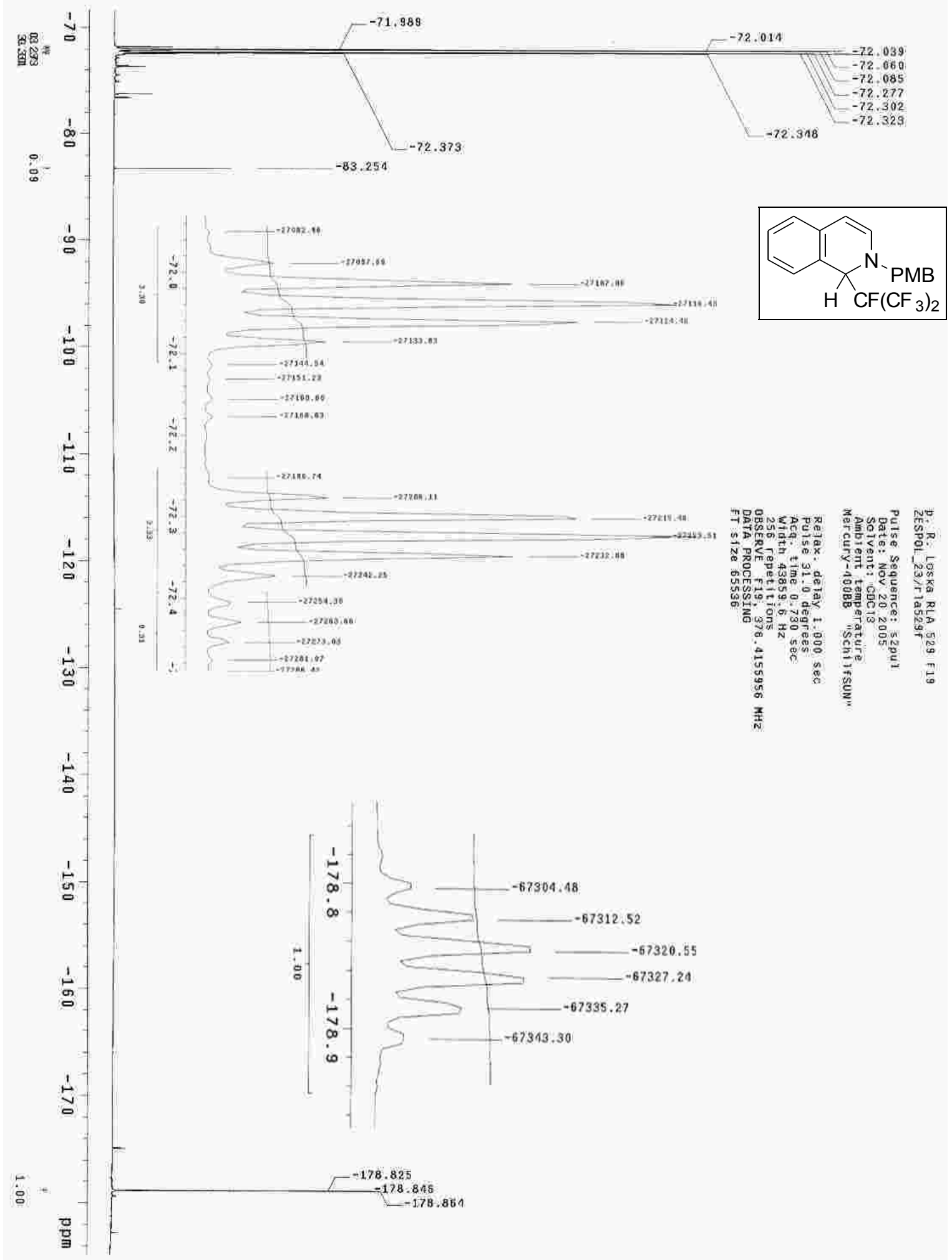




\section{Compound 2e': ${ }^{1} \mathrm{H}$ NMR}

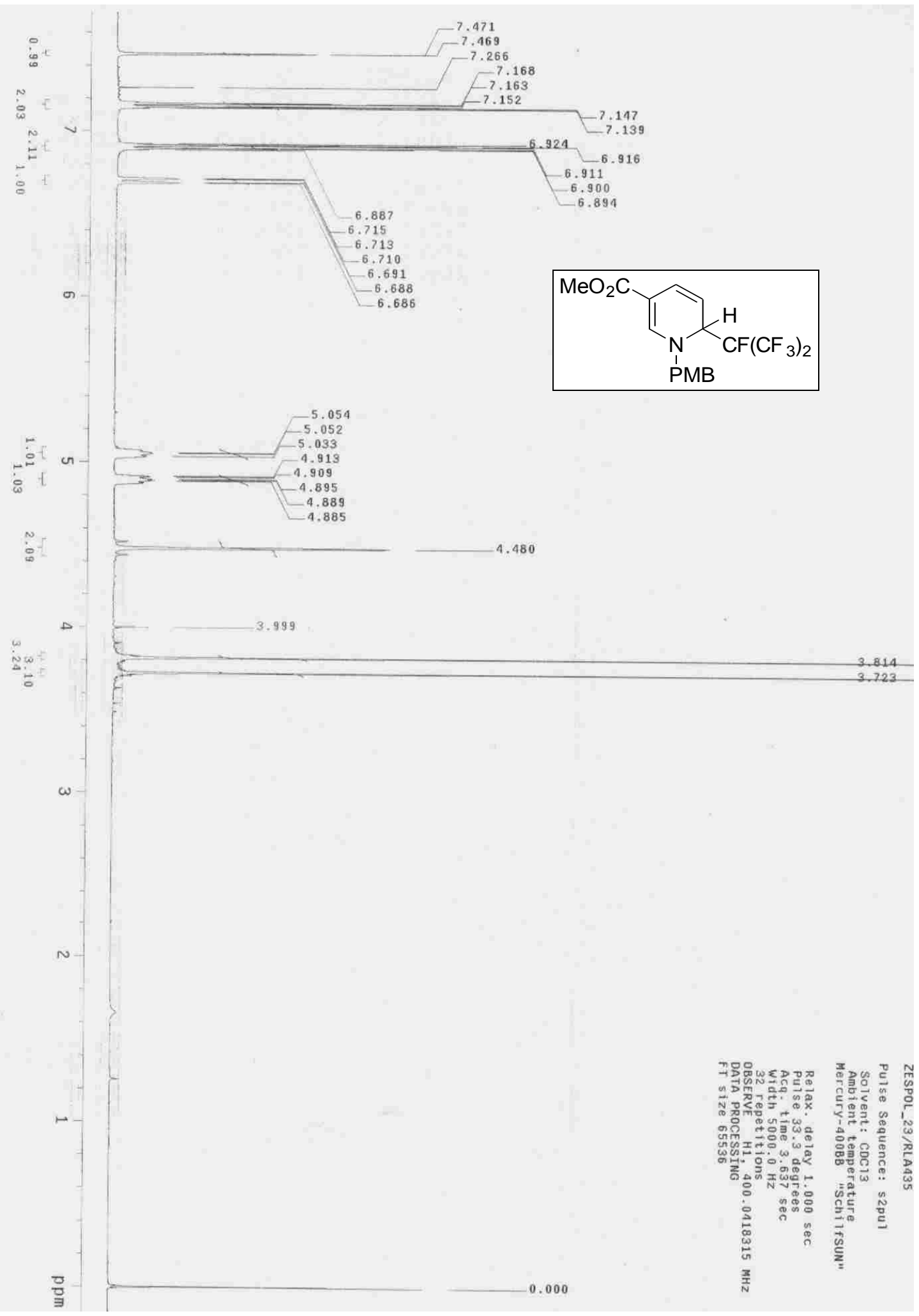




\section{Compound 2e': ${ }^{13} \mathrm{C}$ NMR}

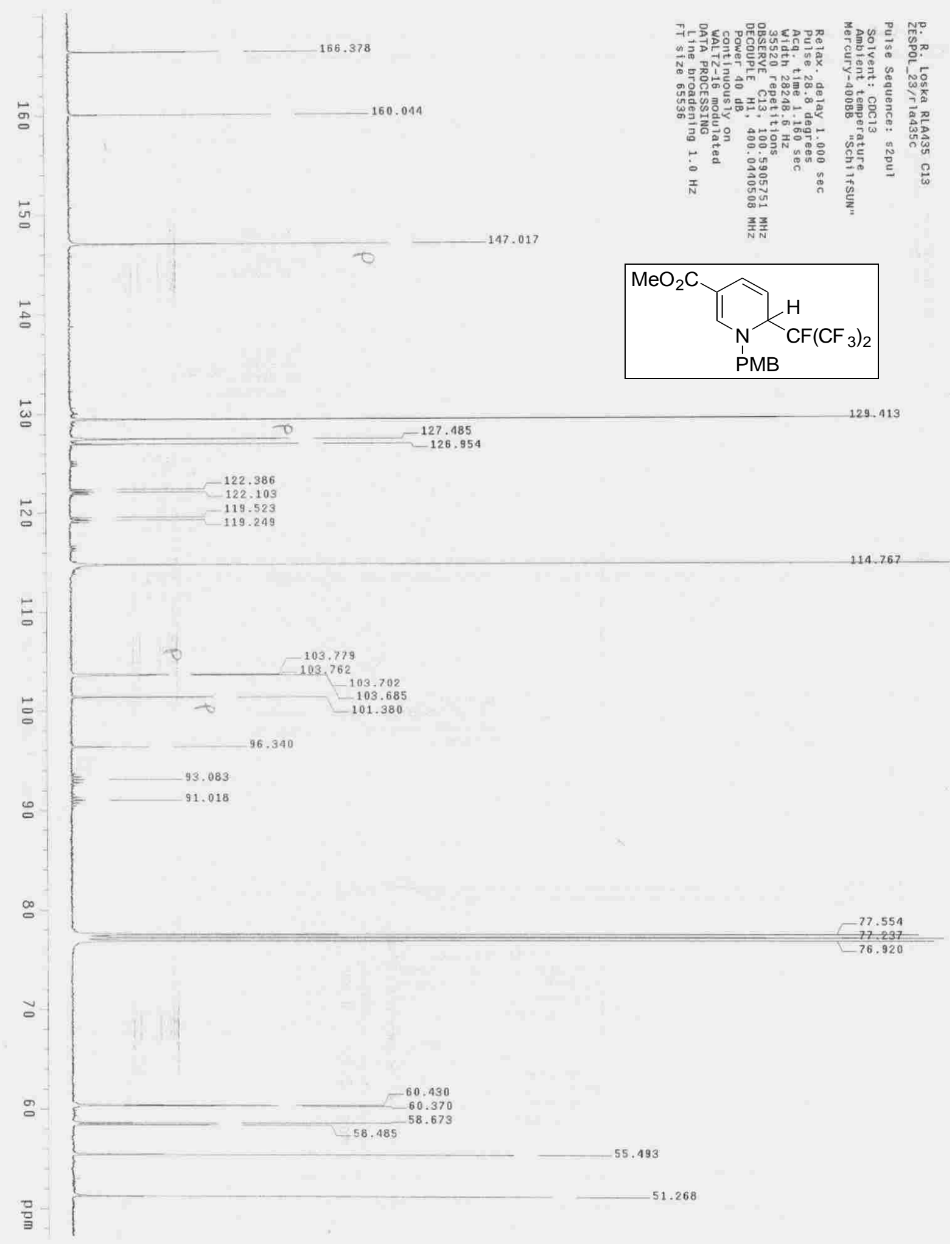


Compound 2e': ${ }^{19}$ F NMR

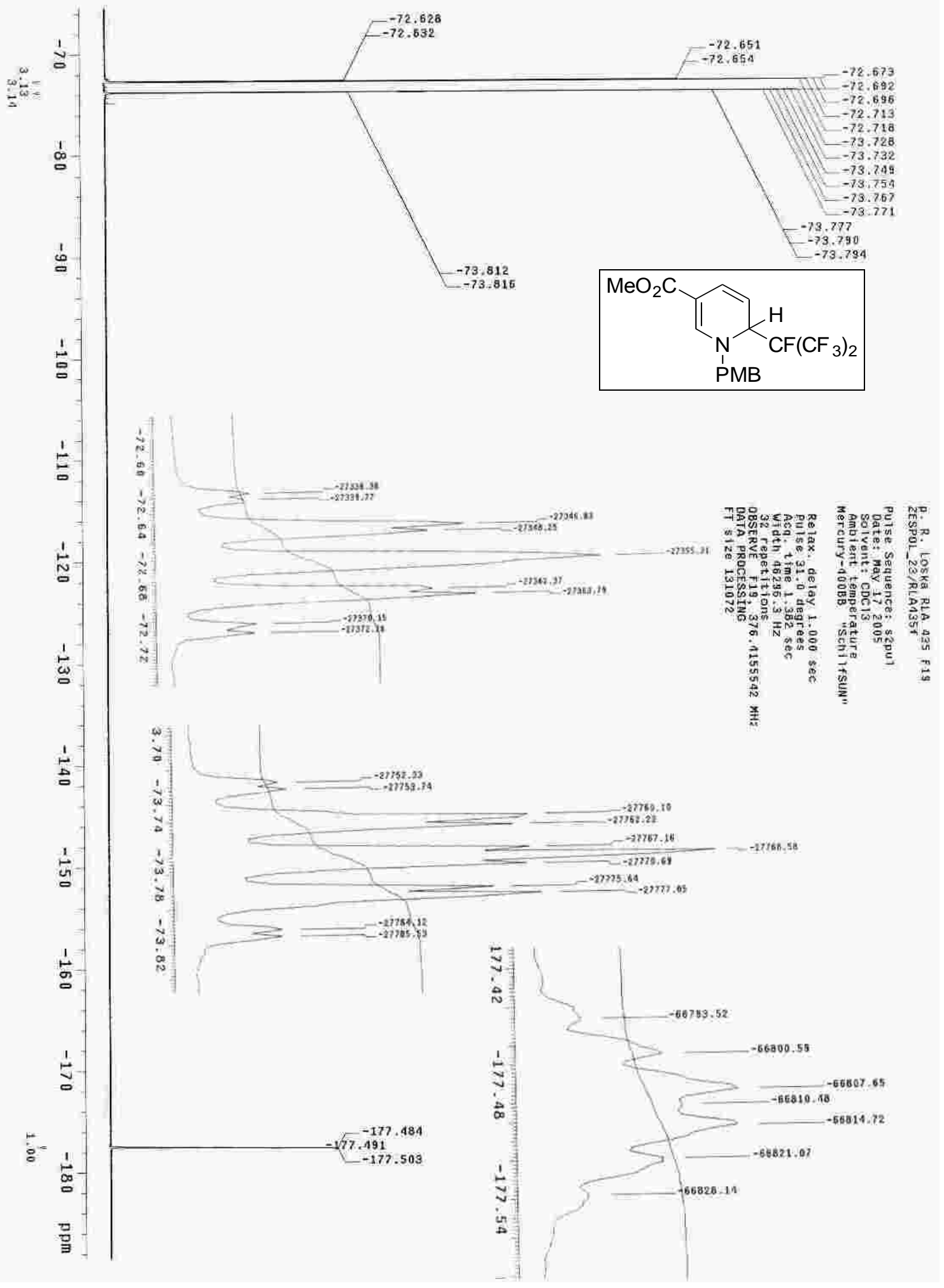




\section{Compound 2e': ${ }^{1} \mathrm{H}-{ }^{1} \mathrm{H}$ COSY}

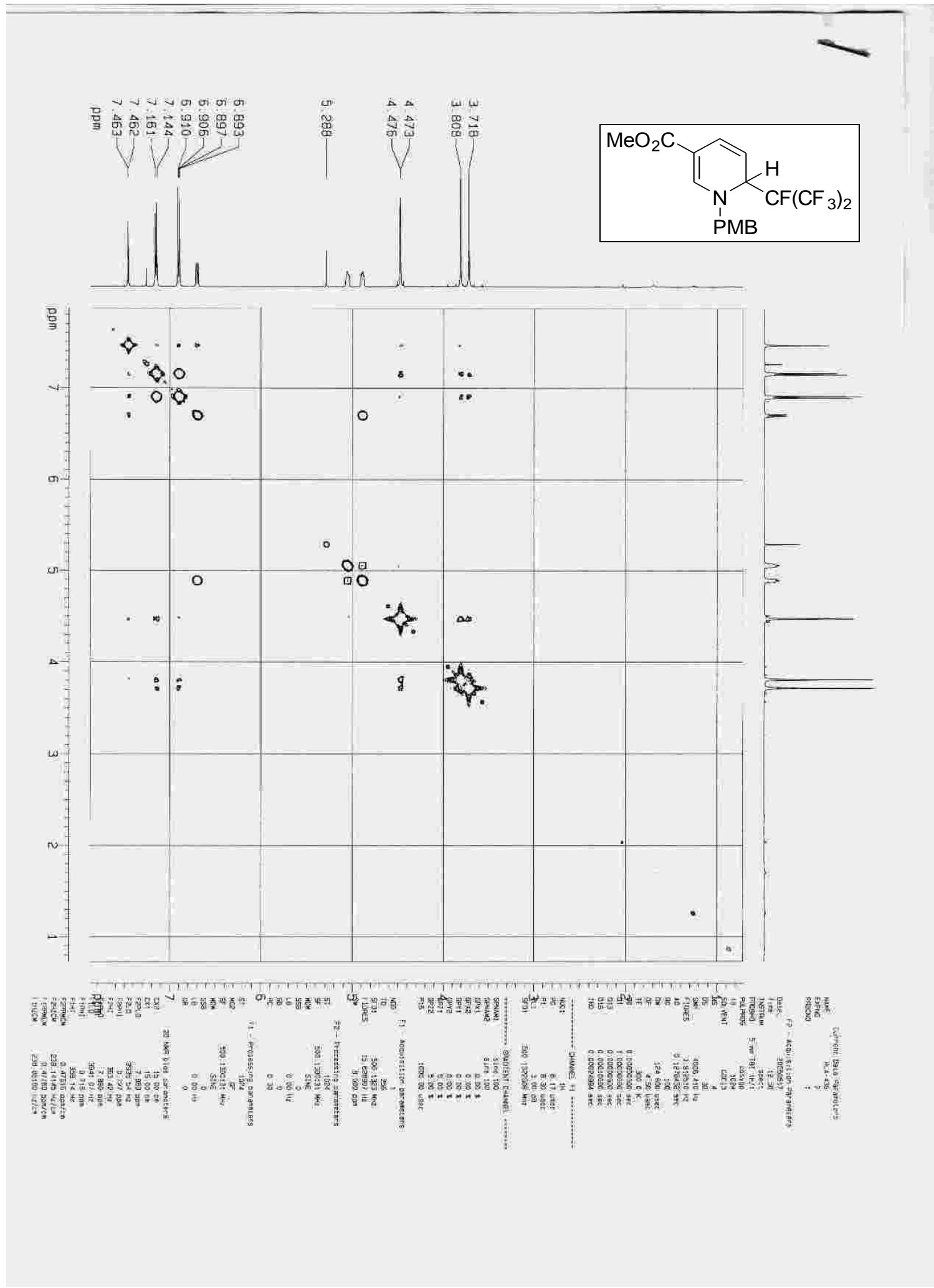




\section{Compound 2e': HSQC}

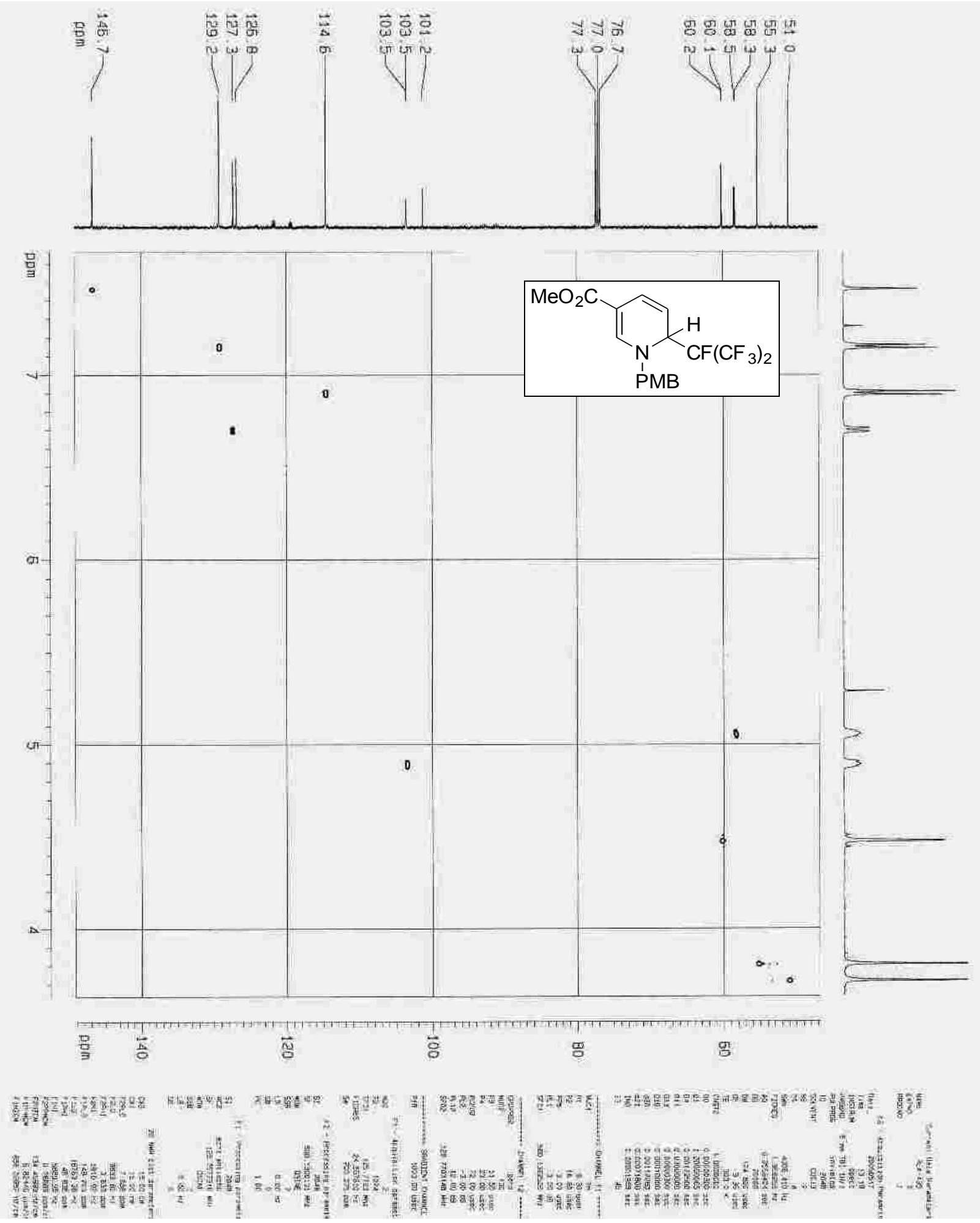




\section{Compound 2e': HMBC}

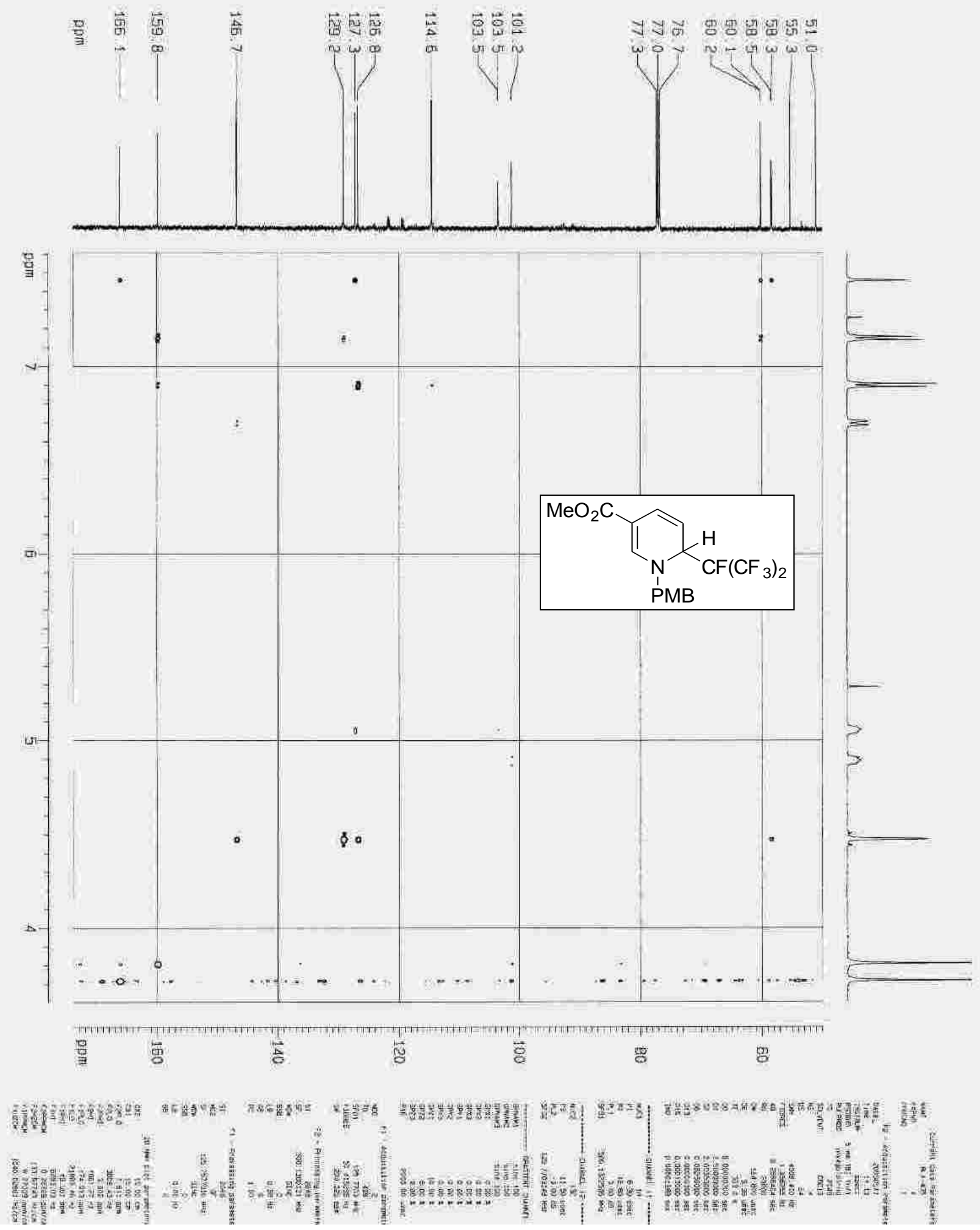




\section{Compound 3e': ${ }^{1} \mathrm{H}$ NMR}

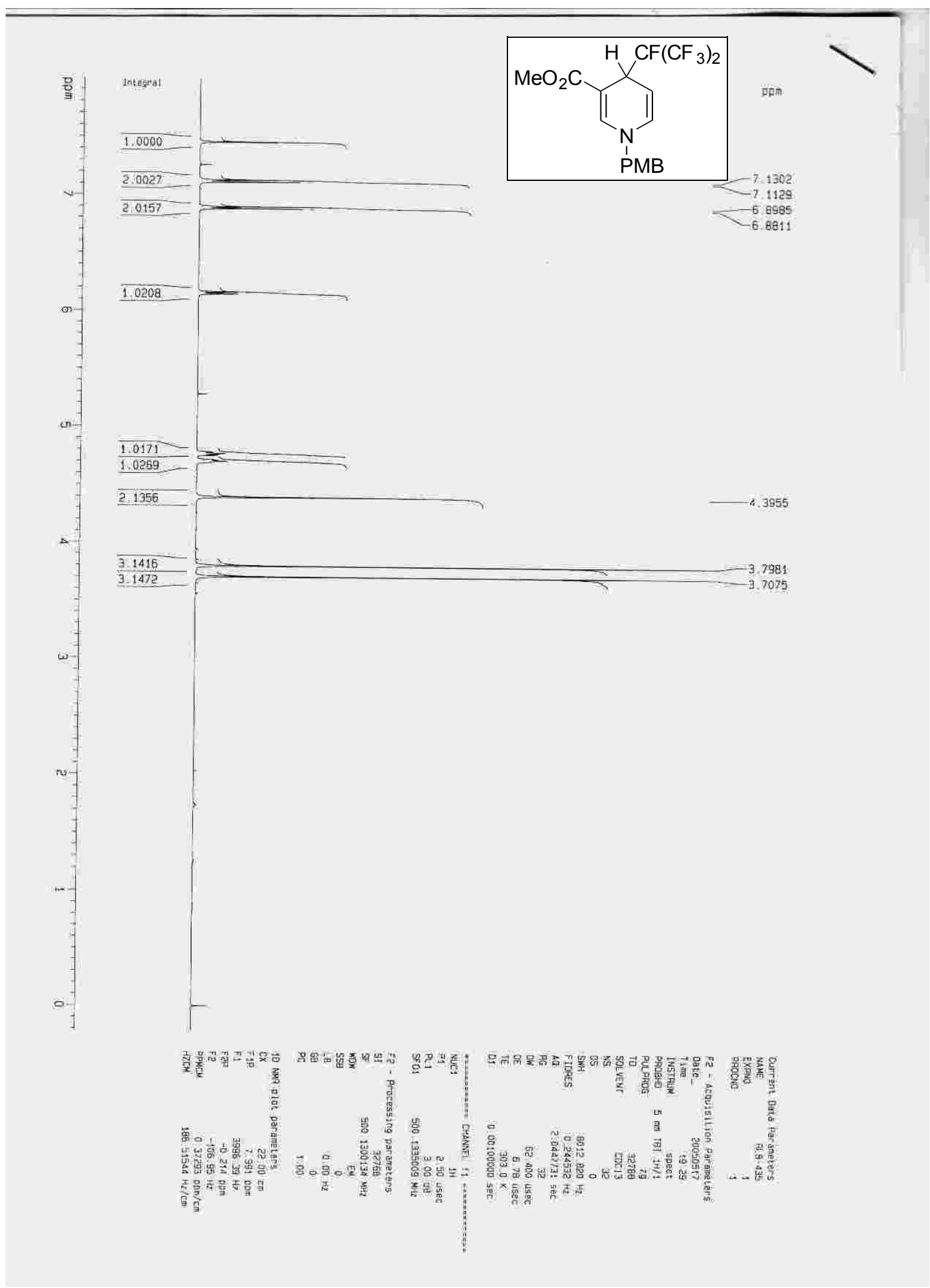




\section{Compound 3e': ${ }^{13} \mathrm{C}$ NMR}

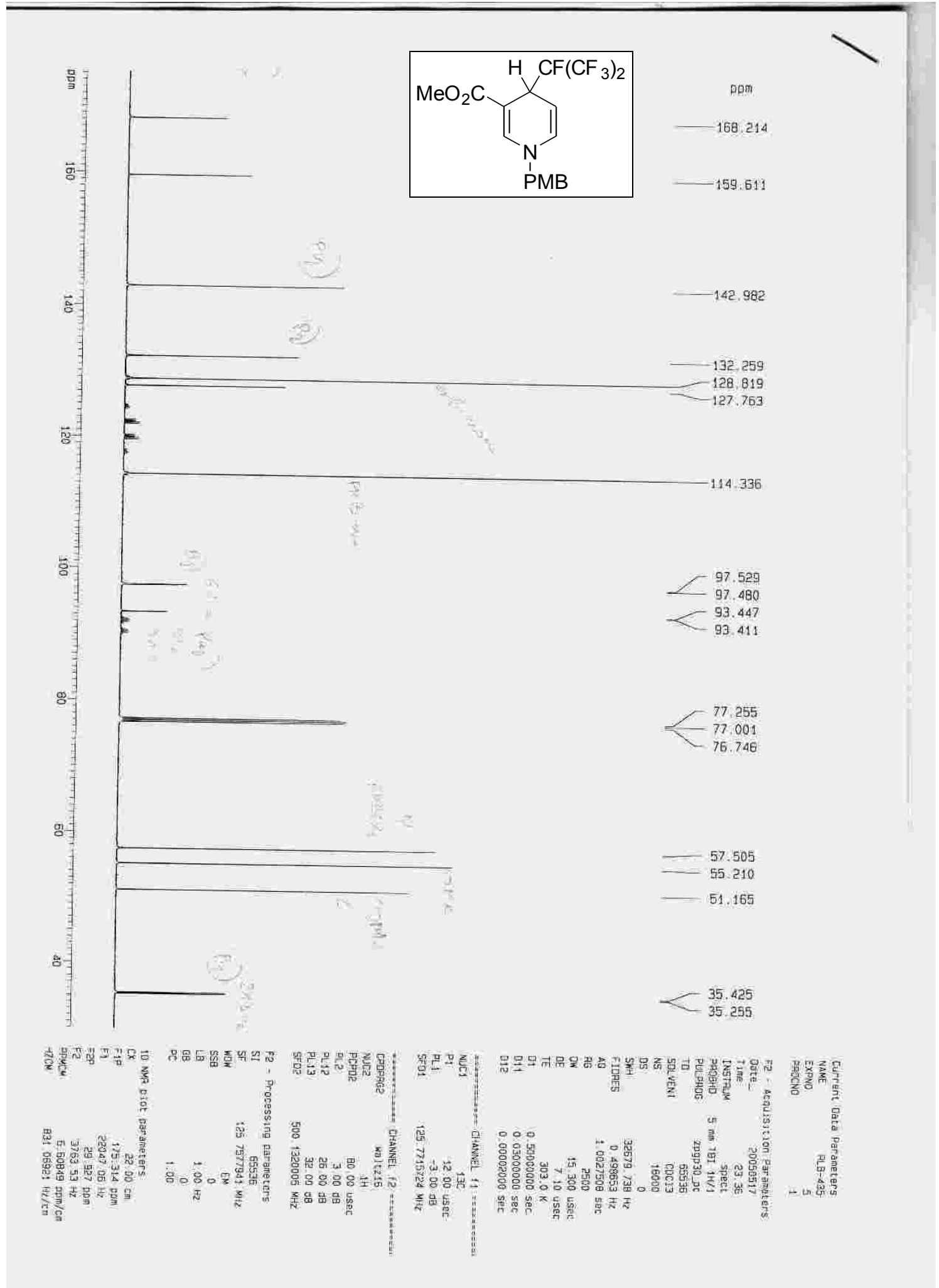




\section{Compound 3e': ${ }^{19} \mathrm{~F}$ NMR}

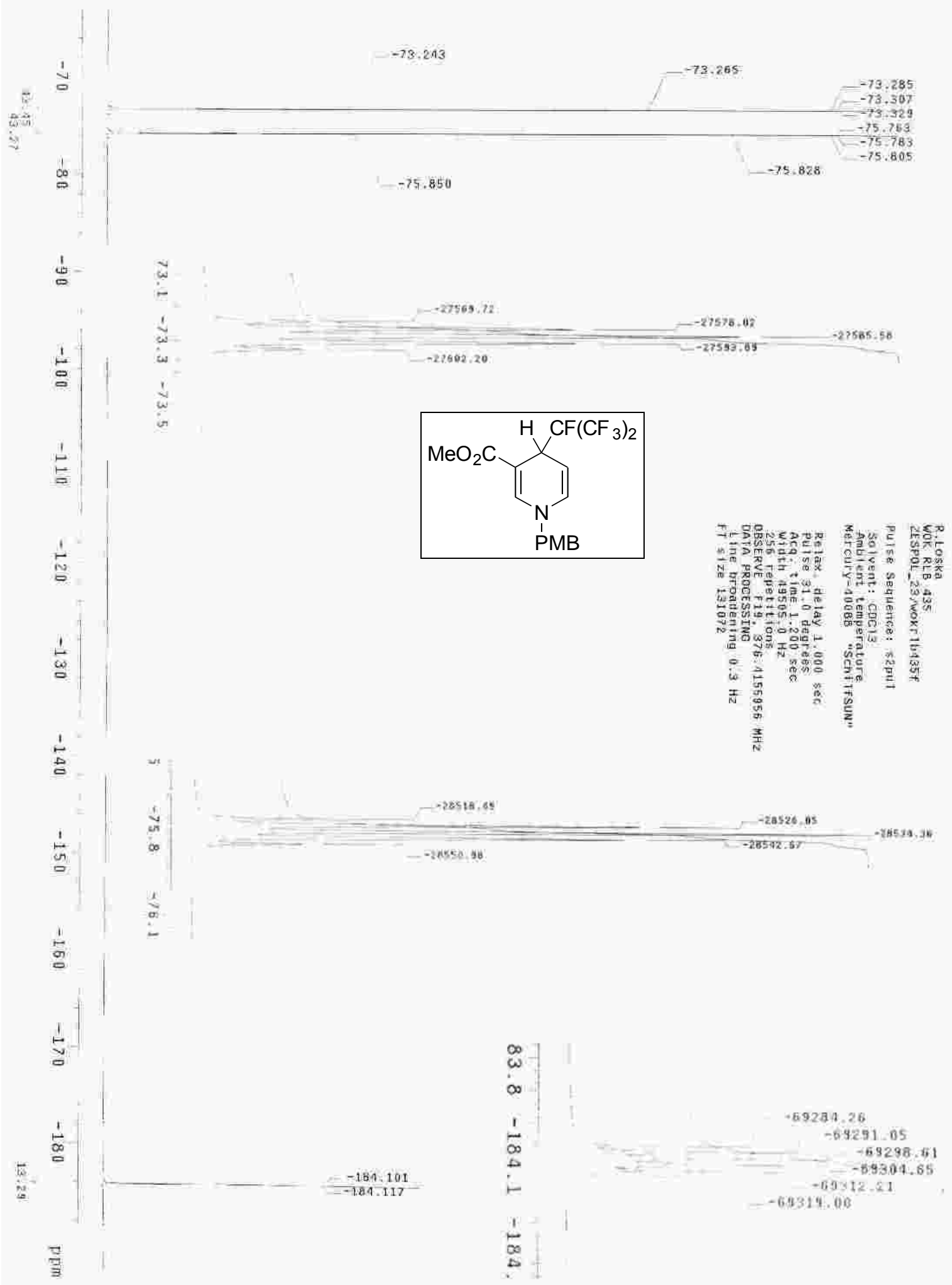




\section{Compound 3e': ${ }^{1} \mathrm{H}-{ }^{1} \mathrm{H}$ COSY}

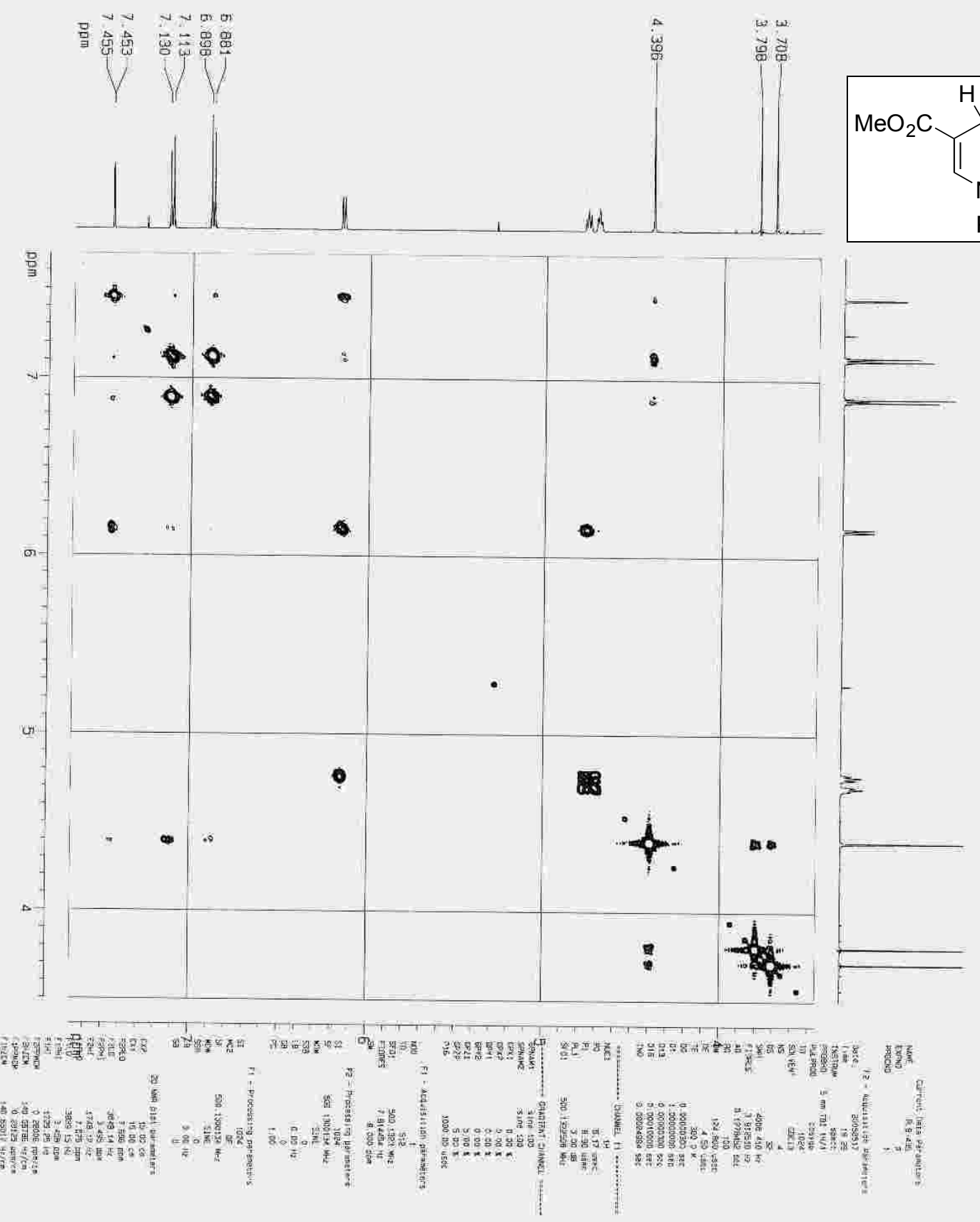




\section{Compound 3e': HSQC}

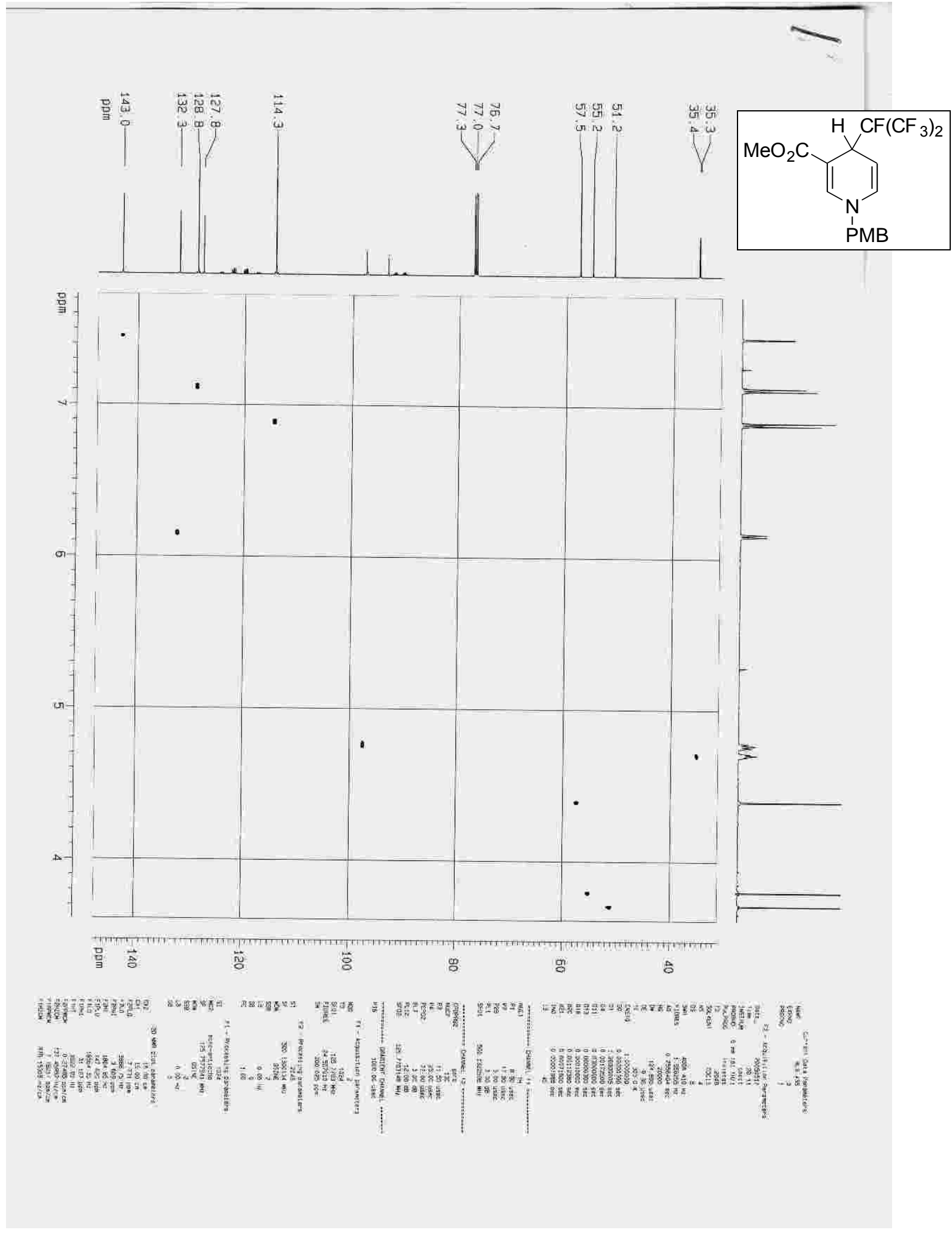




\section{Compound 3e': HMBC}

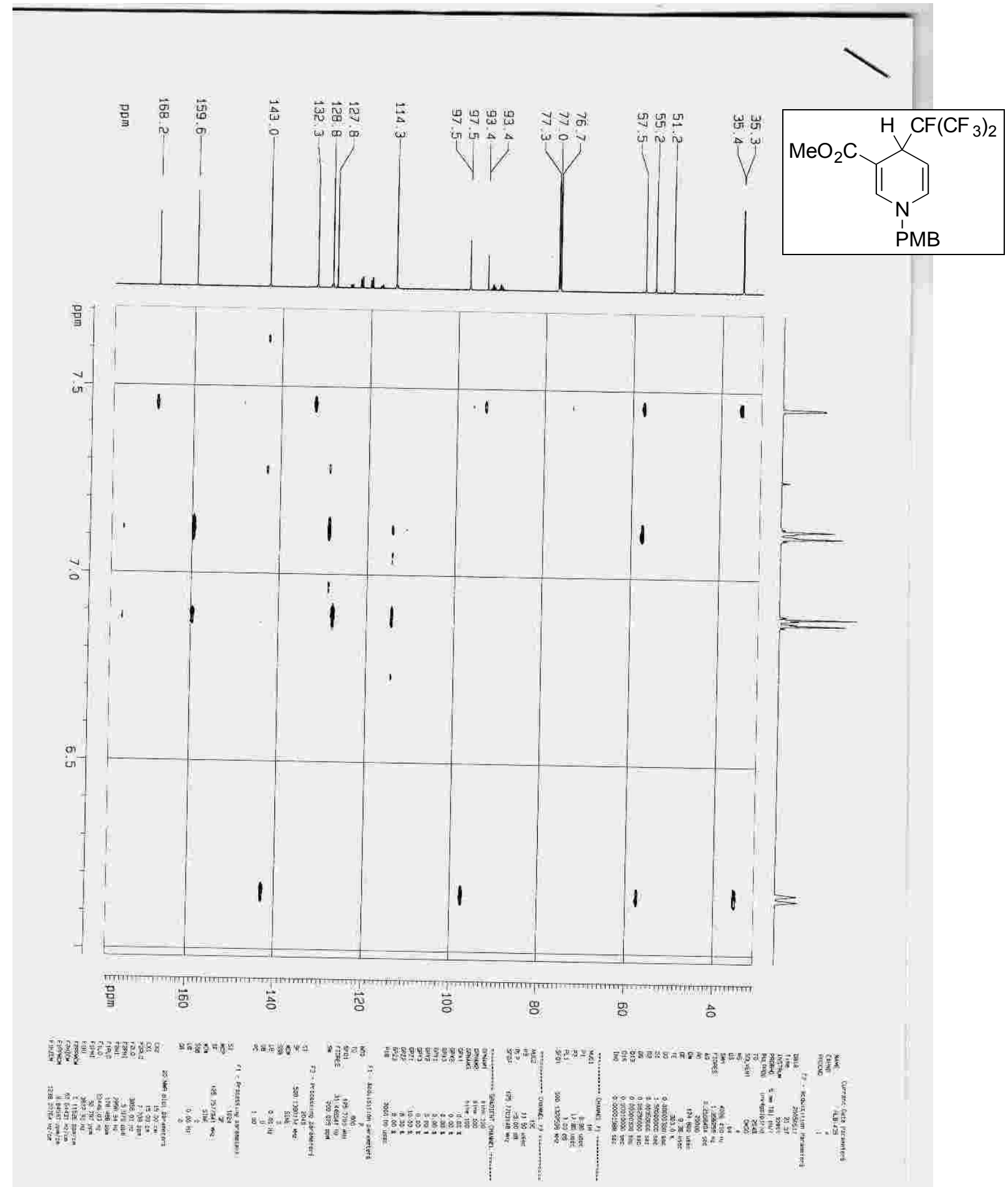




\section{Compound 2f': ${ }^{1} \mathrm{H}$ NMR}

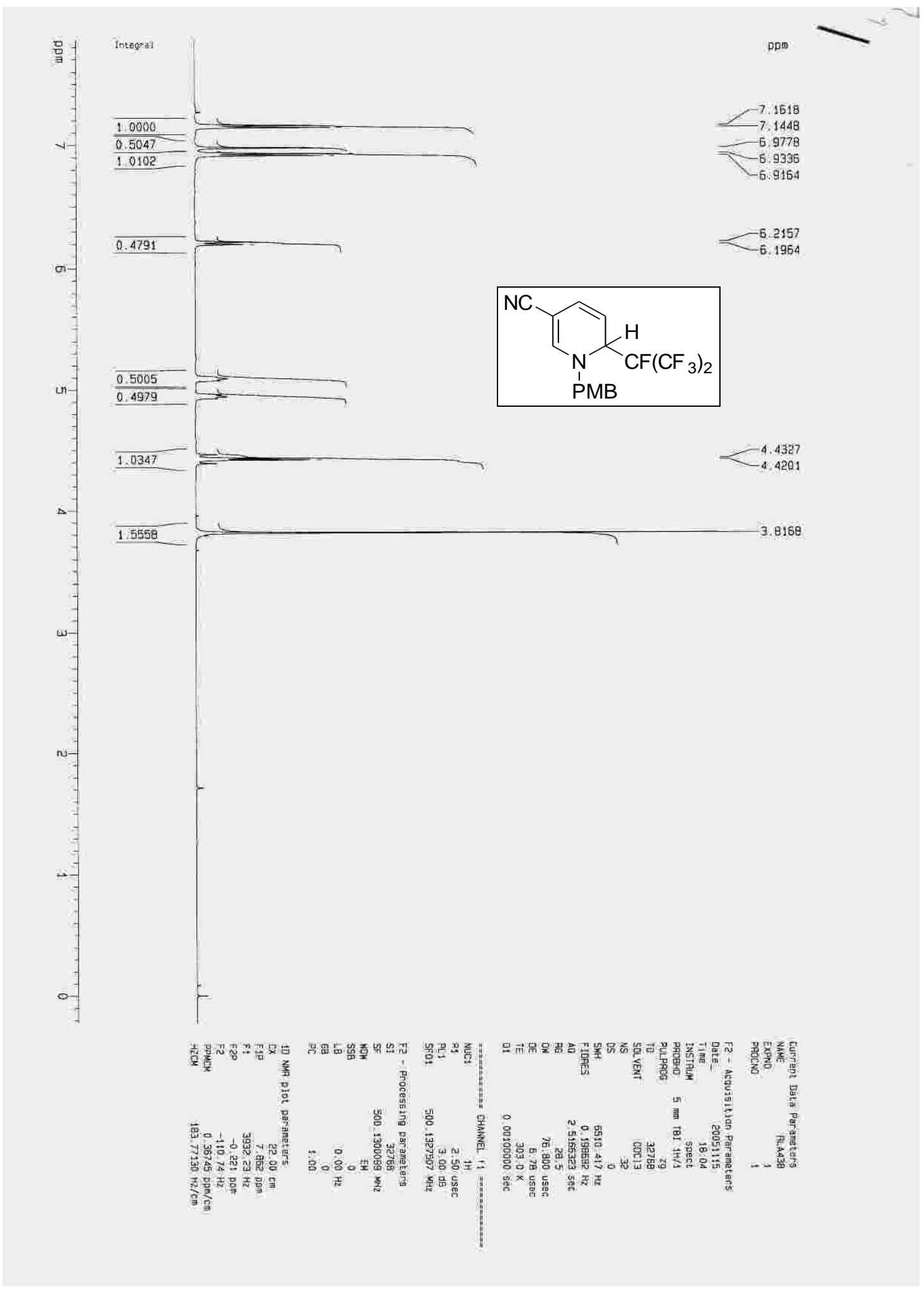




\section{Compound 2f': ${ }^{13} \mathrm{C}$ NMR}

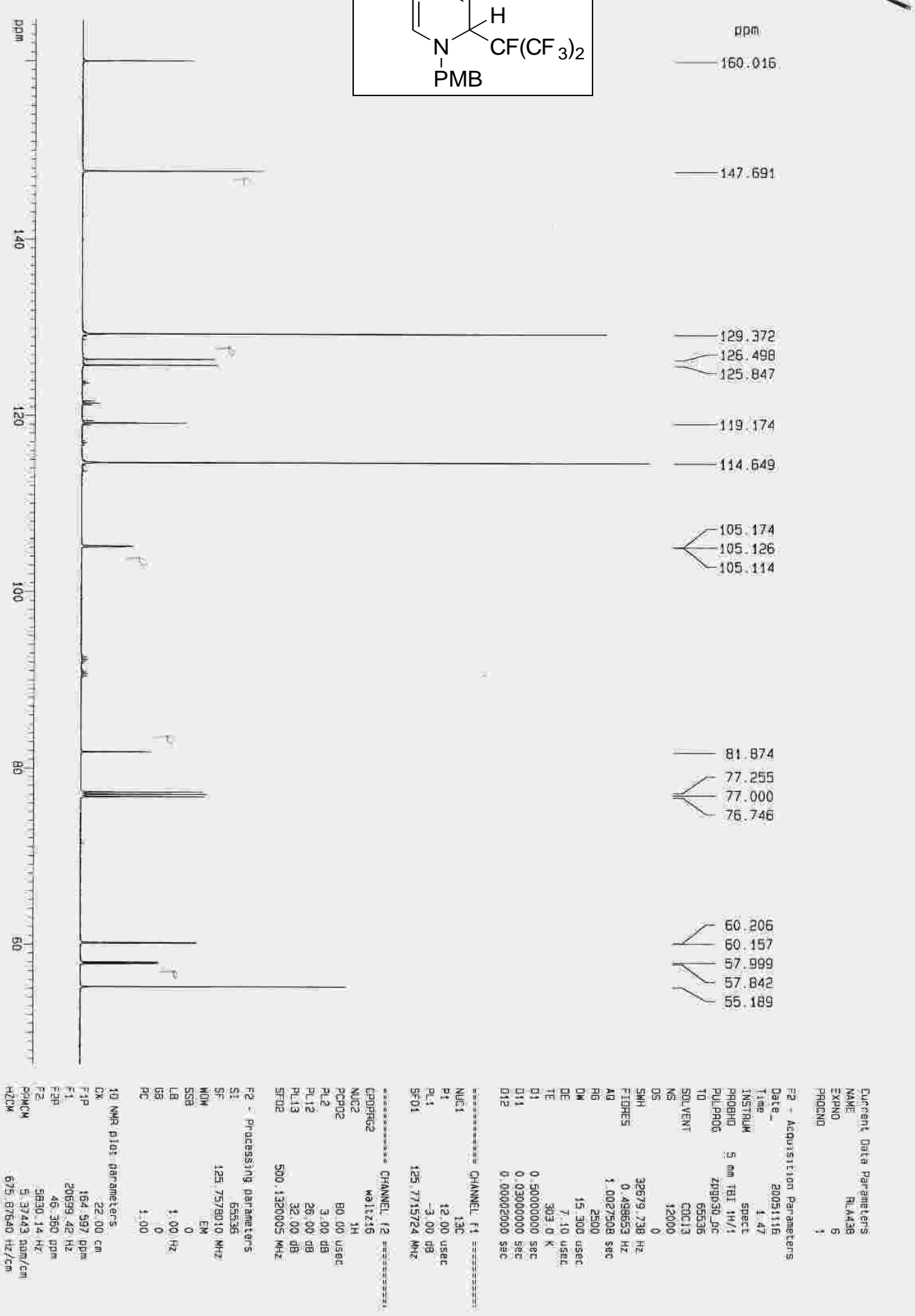




\section{Compound 2f': ${ }^{19}$ F NMR}

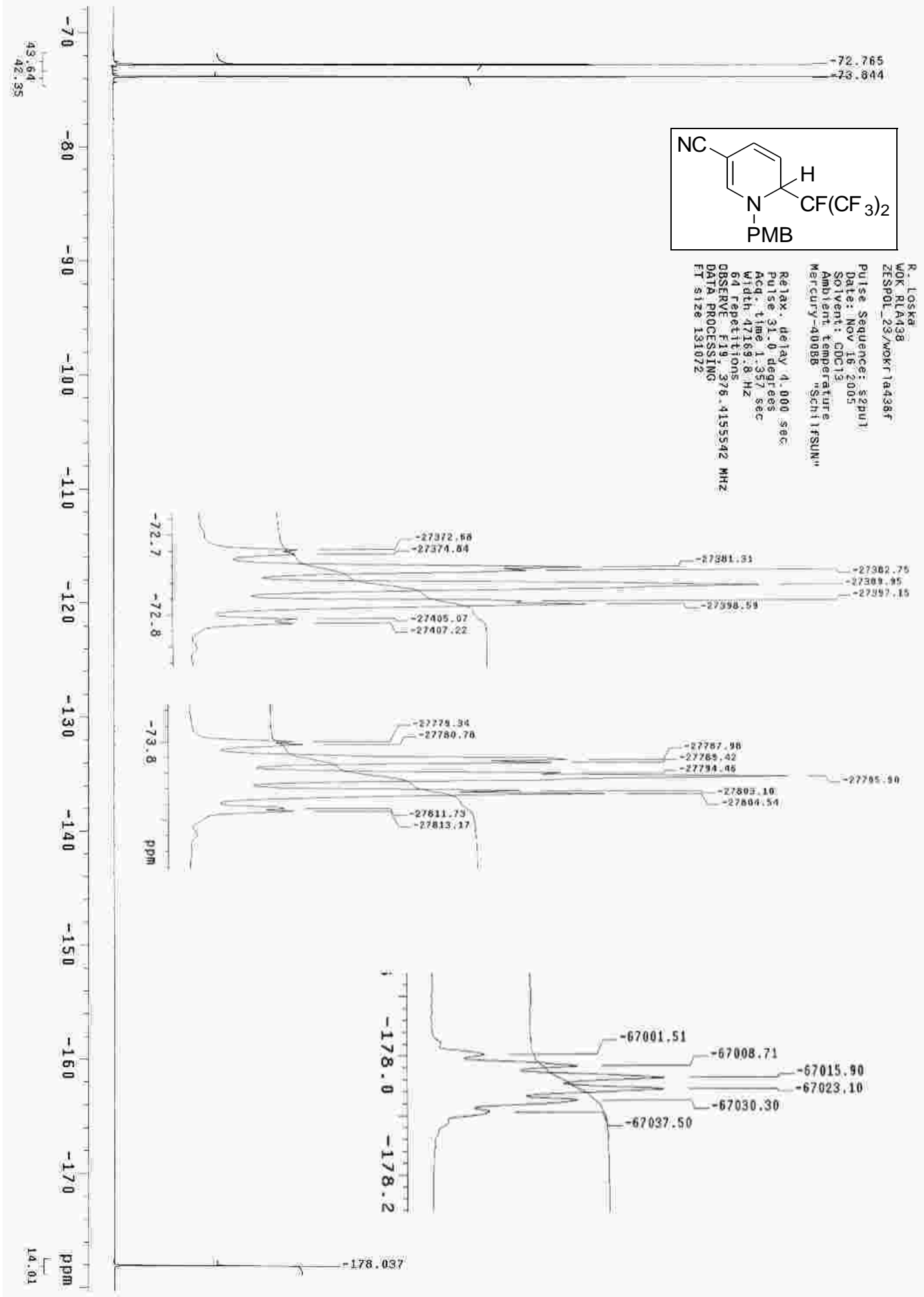




\section{Compound 3f': ${ }^{1} \mathrm{H}$ NMR}
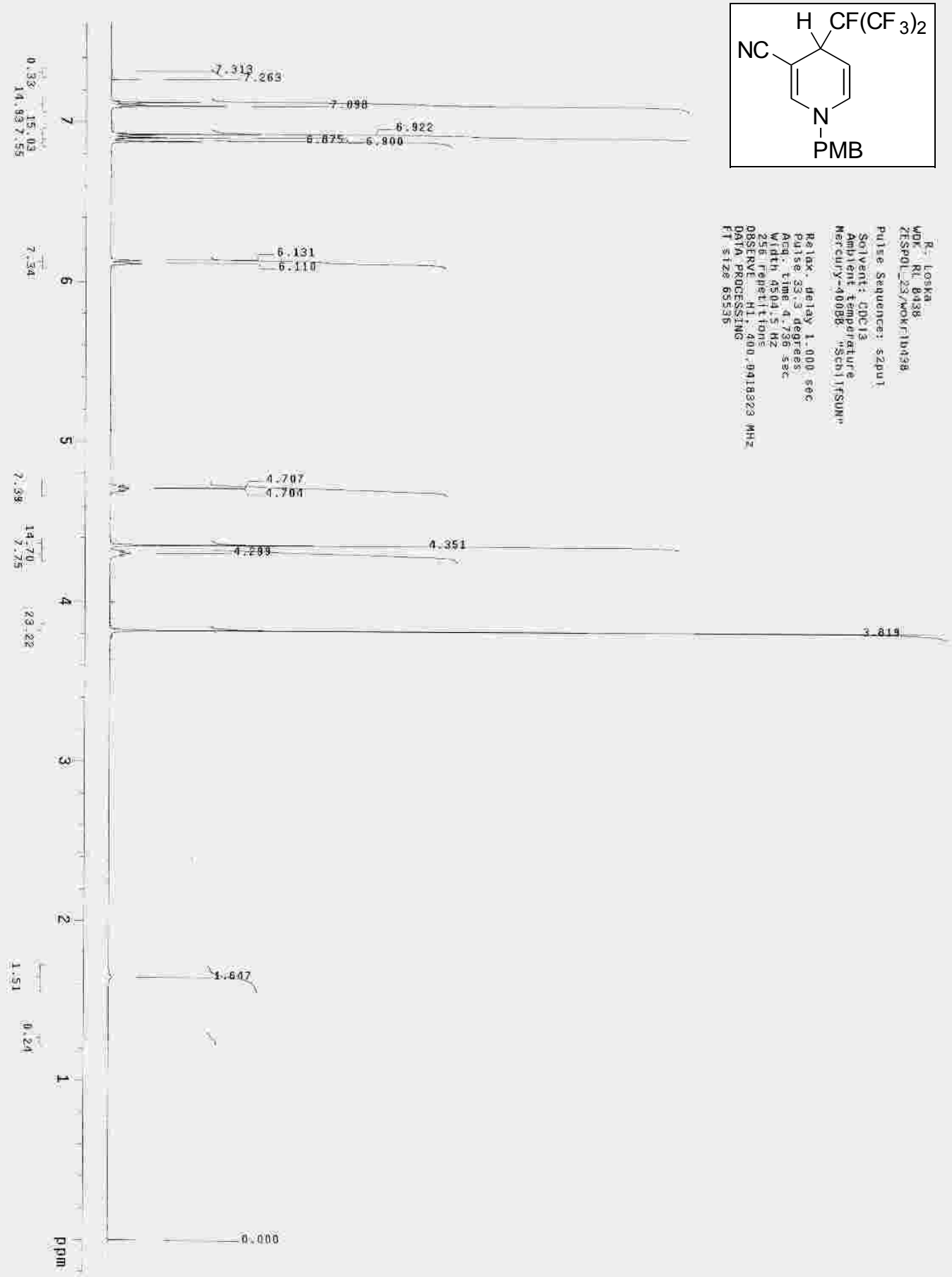


\section{Compound 3f': ${ }^{13} \mathrm{C}$ NMR}

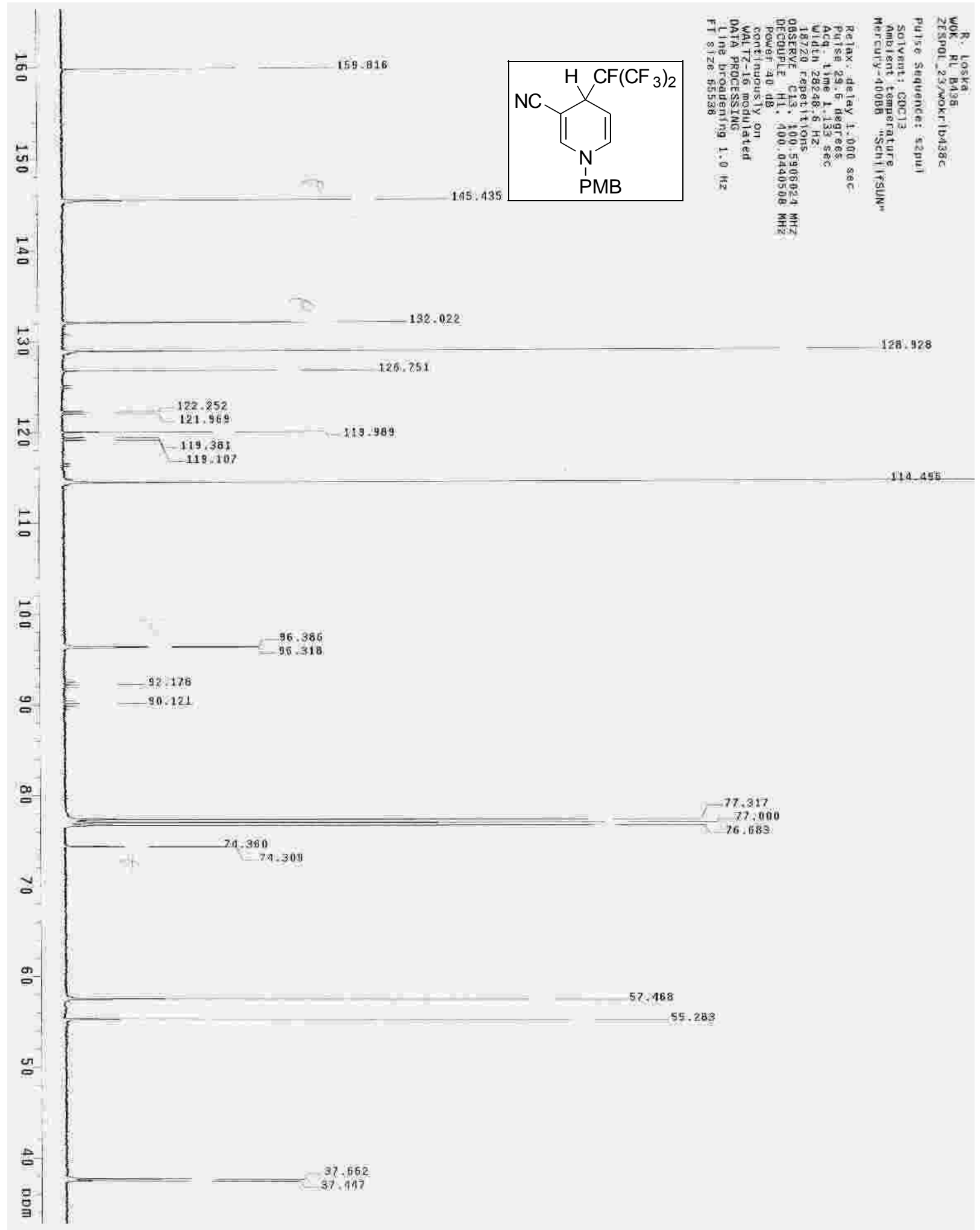




\section{Compound 3f': ${ }^{19}$ F NMR}

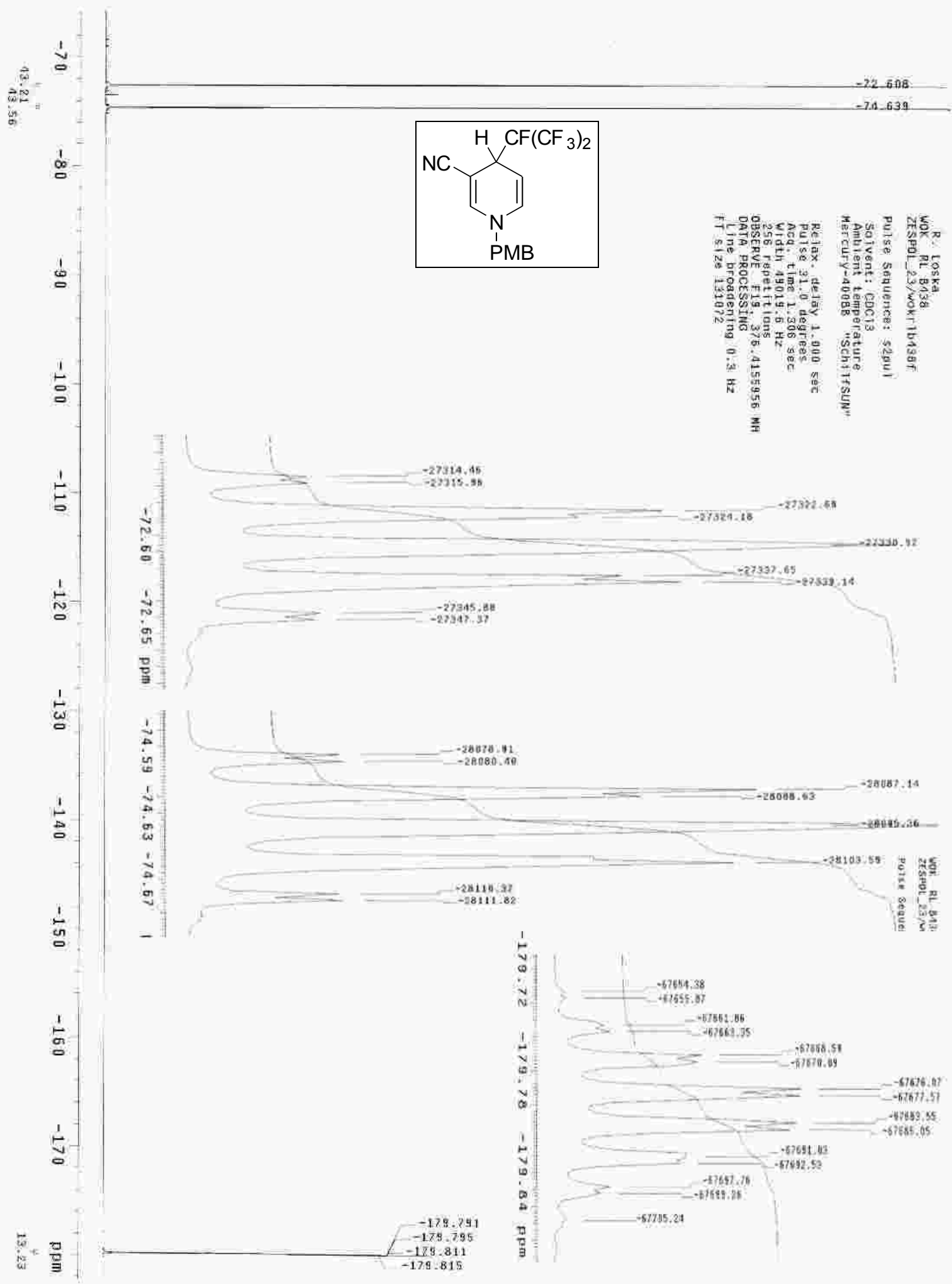




\section{Compounds 2g' and 3g': ${ }^{1} \mathrm{H}$ NMR}

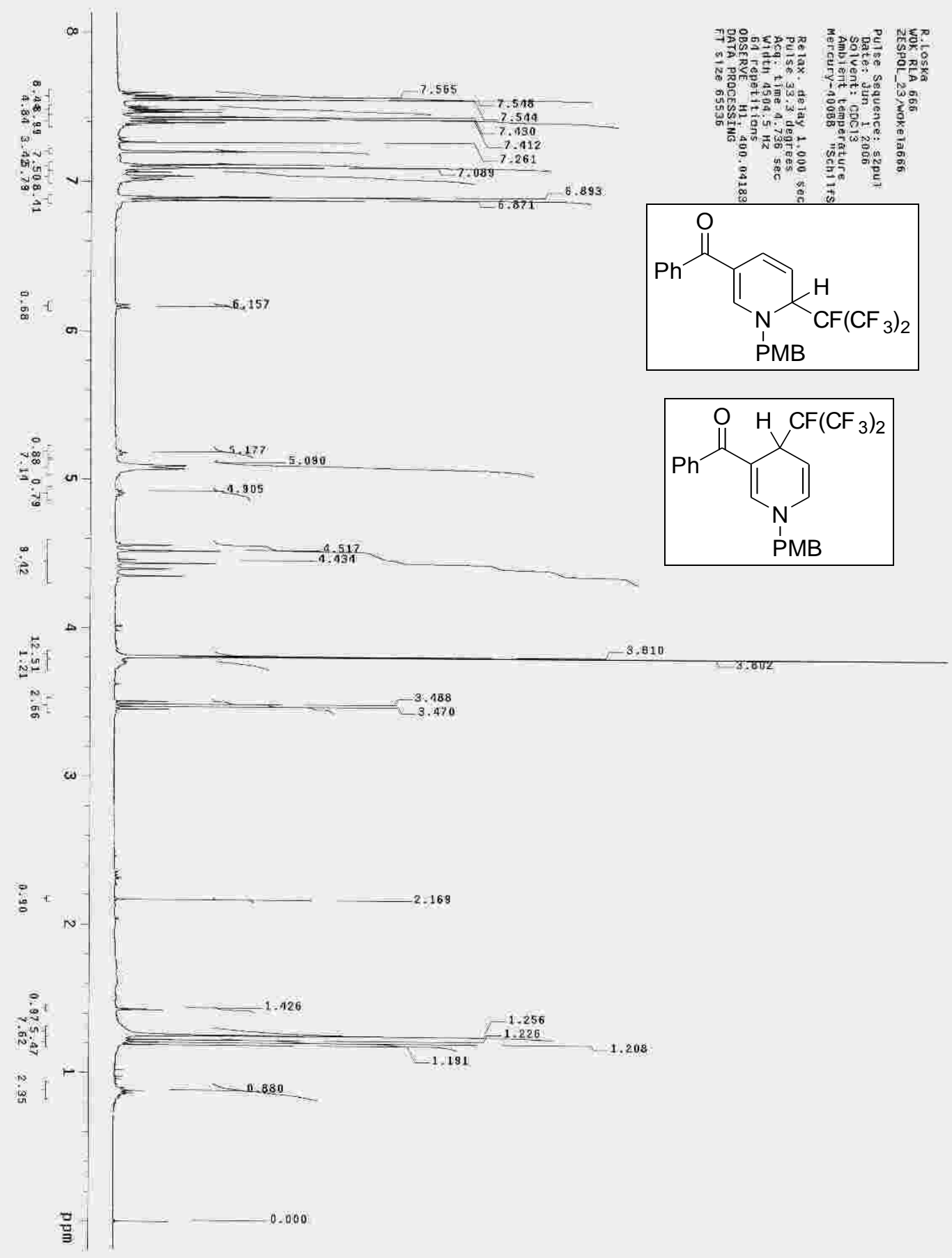




\section{Compounds 2g' and 3g': ${ }^{13} \mathrm{C}$ NMR}

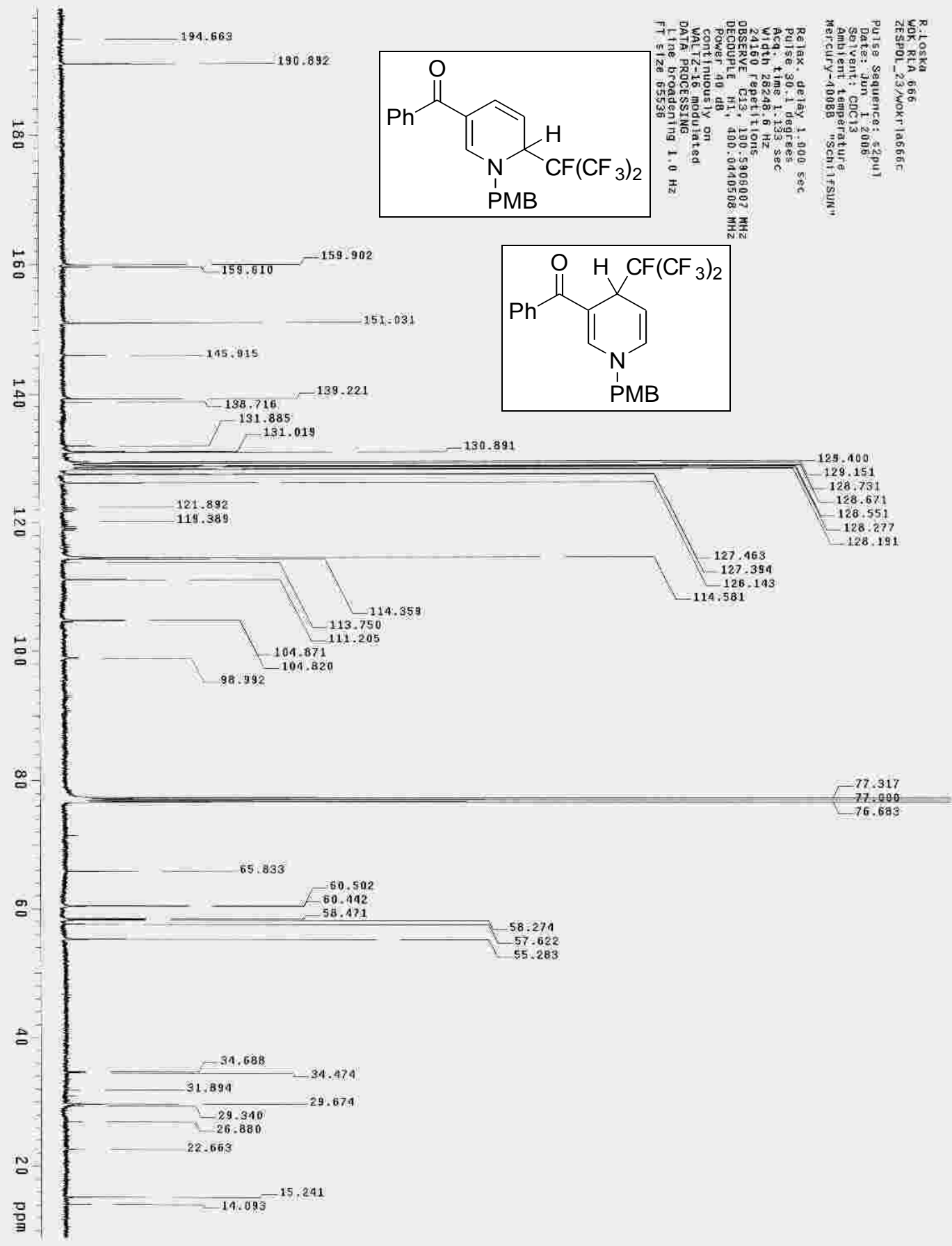




\section{Compounds 2 $\mathbf{g}^{\prime}$ and $\mathbf{3} \mathbf{g}^{\prime}:{ }^{19} \mathrm{~F}$ NMR}

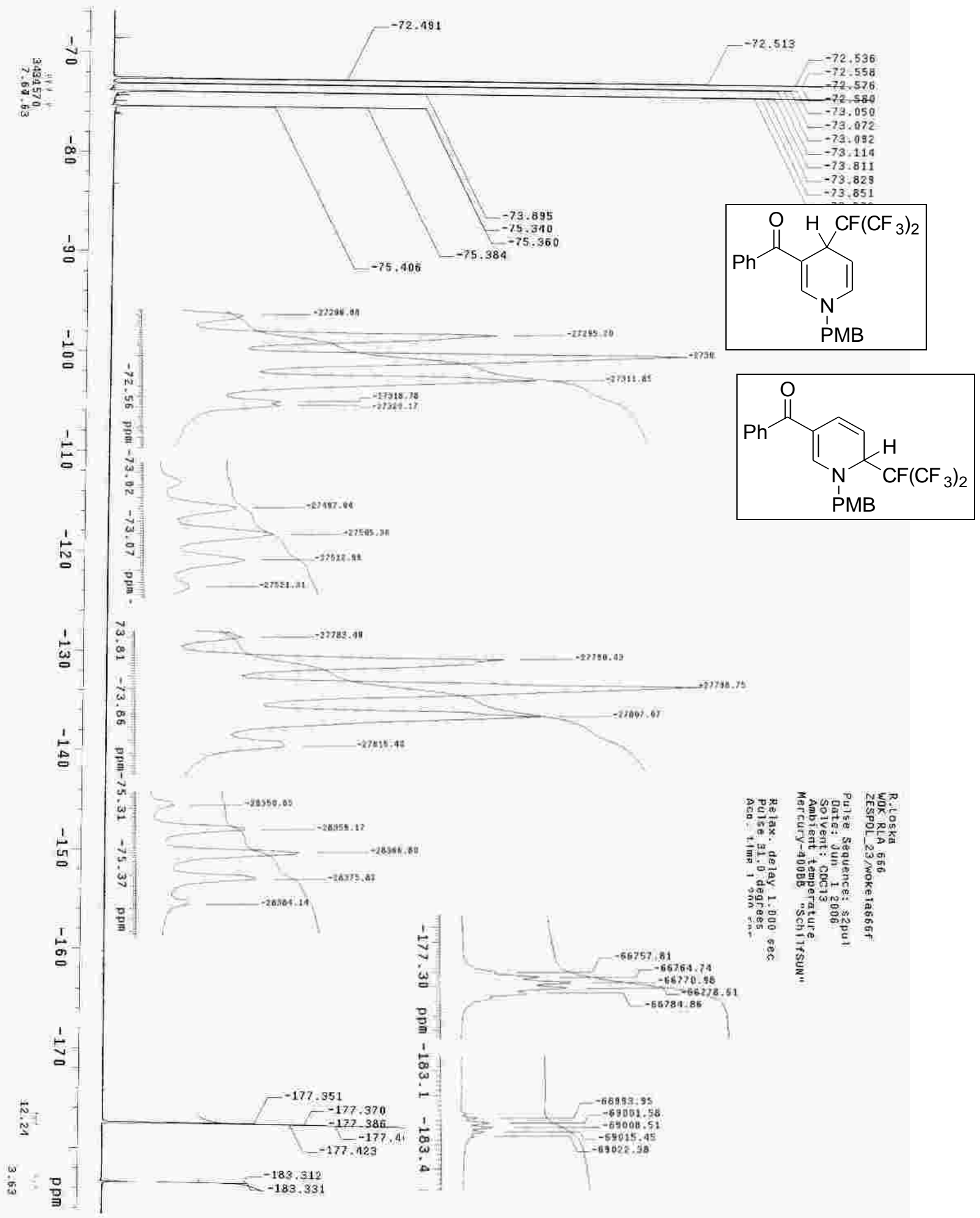




\section{Compound 3h': ${ }^{1} \mathrm{H}$ NMR}

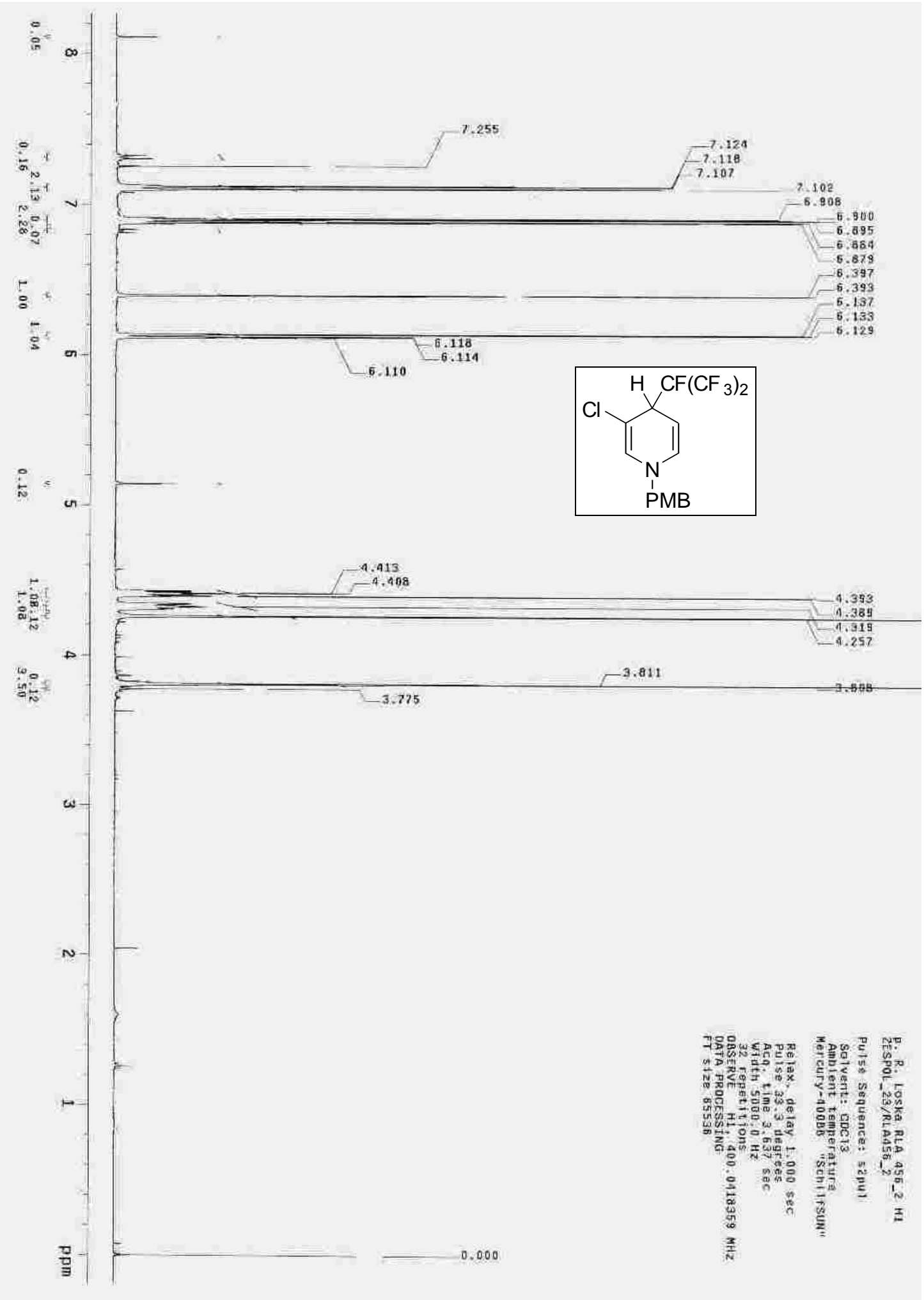




\section{Compound 3h': ${ }^{13} \mathrm{C}$ NMR}

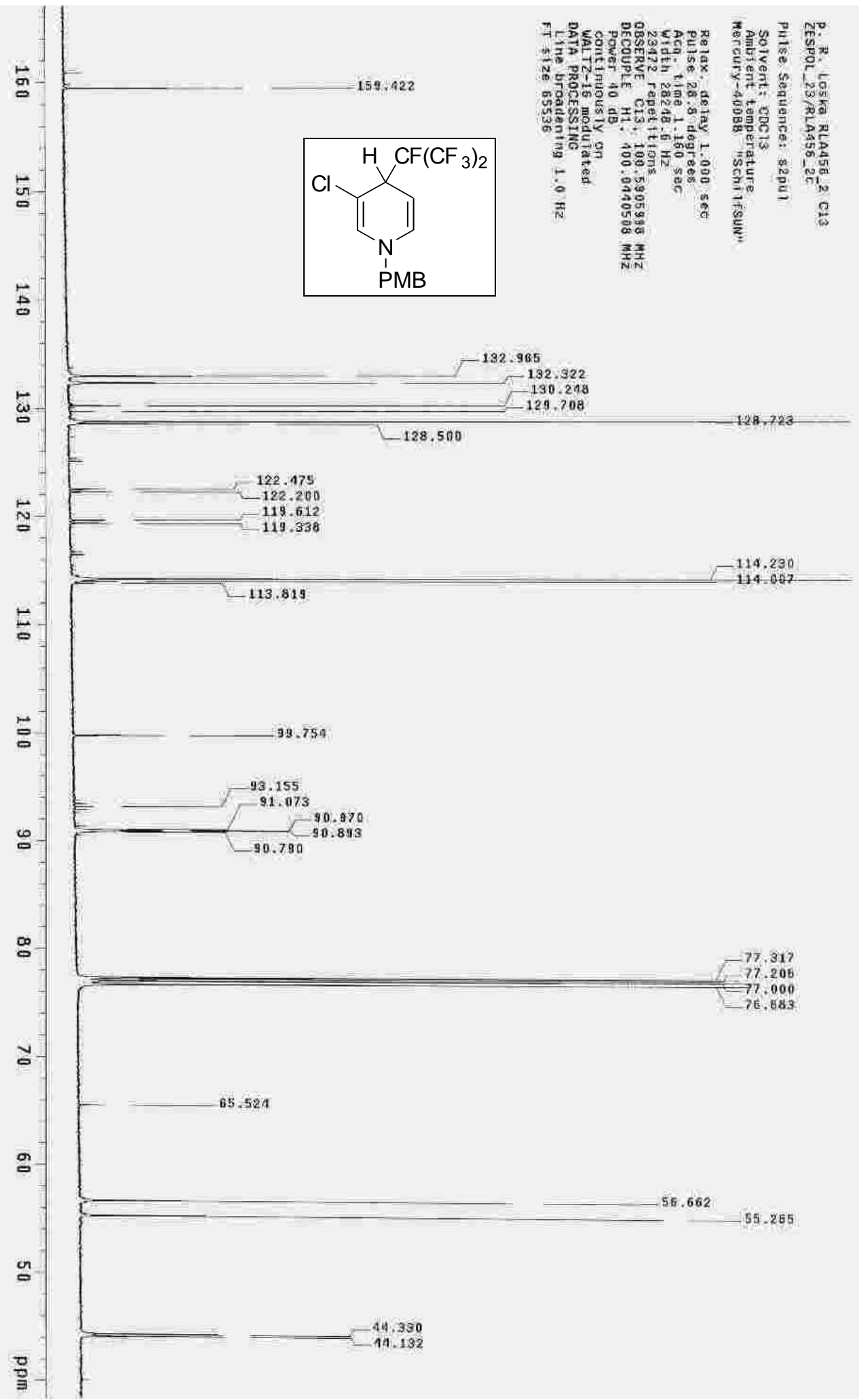




\section{Compound 3h': ${ }^{19}$ F NMR}

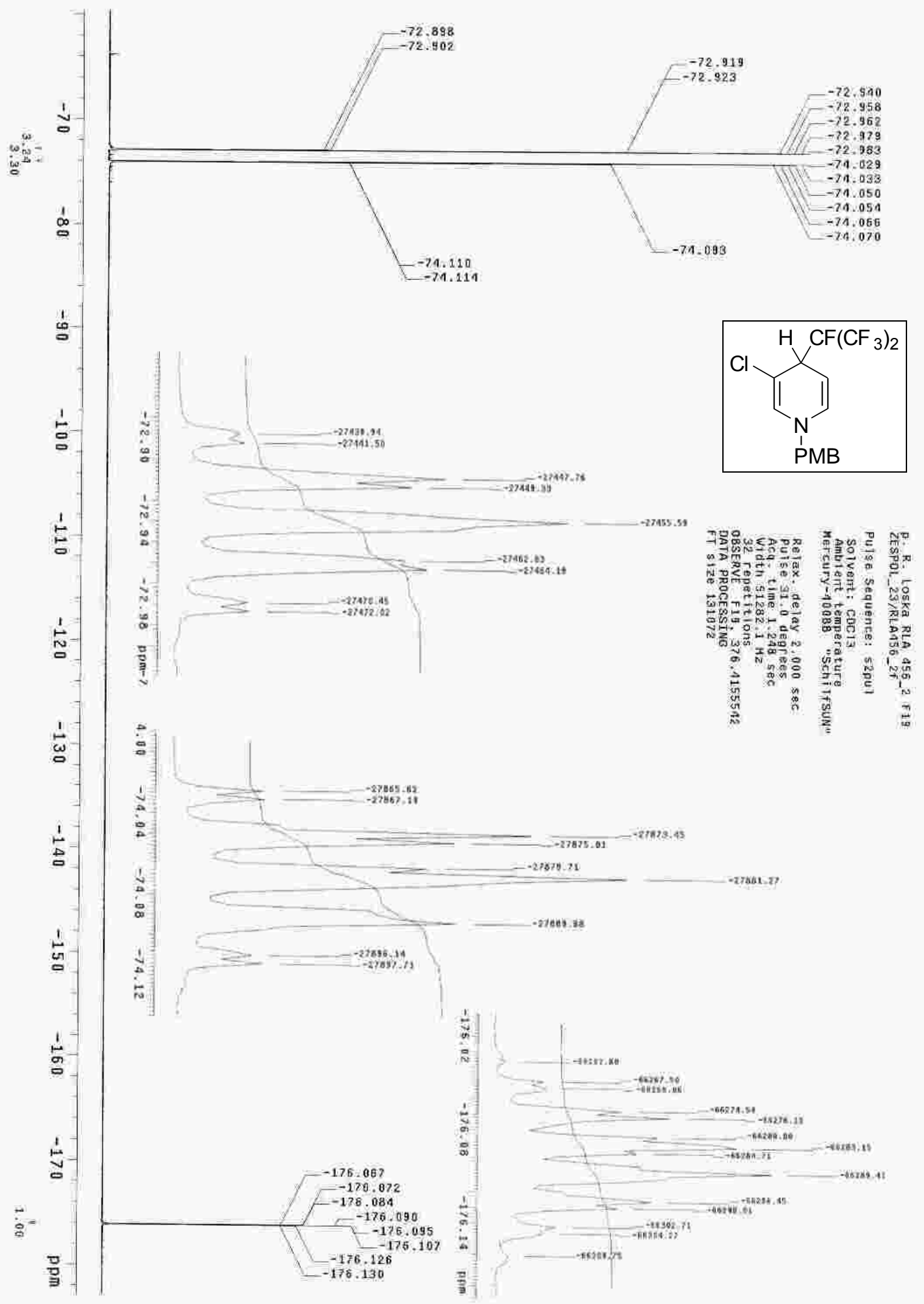

